\title{
Conformal Field Theory and Torsion Elements of the Bloch Group
}

\author{
Werner Nahm \\ Dublin Institute for Advanced Studies wnahm@stp.dias.ie
}

\begin{abstract}
Summary. We argue that rational conformally invariant quantum field theories in two dimensions are closely related to torsion elements of the algebraic K-theory group $K_{3}(\mathbb{C})$. If such a theory has an integrable matrix perturbation with purely elastic scattering matrix, then the partition function has a canonical sum representation. Its asymptotic behaviour is given in terms of the solution of an algebraic equation which can be read off from the scattering matrix. The solutions yield torsion elements of an extension of the Bloch group which seems to be equal to $K_{3}(\mathbb{C})$. These algebraic equations are solved for integrable models given by arbitrary pairs of equations are solved for integrable models given by arbitrary pairs of A-type Cartan matrices. The paper should be readable by mathematicians.
\end{abstract}

\section{Introduction}

We will study a relation between certain integrable quantum field theories in two dimensions and the algebraic $K$-theory of the complex numbers. As was acknowledged in the first publication [NRT93, discussions with A. Goncharov, A.N. Kirillov and D. Zagier were essential, and the results appear to be well worth of the common attention of physicists and mathematicians. Some developments in physics and to a lesser degree in mathematics were stimulated, but progress was slow and a fresh start by young researchers in both fields may be in order.

For a long time few mathematicians attempted to cope with the obscurities of quantum field theory, but this has started to change. In particular, the much studied vertex operator algebras capture many features of the conformally invariant quantum field theories in two dimensions. The present investigation will take us a small step farther, since we have to consider integrable massive perturbations of these conformally invariant field theories (CFTs). On the other hand, only rational CFTs will be investigated. For theories with continuous parameters, only special points in their moduli space can be rational, but those points may allow a more complete understanding than generic ones and 
can serve to explore a neighbourhood in CFT moduli space by perturbation theory.

Physicists have a tradition of openness to all kind of mathematics, but algebraic $K$-theory has not been very popular, in part because its definition is very abstract. Much of it is captured by the Bloch group, however, which is easy to work with. The Bloch group $B(K)$ of a field $K$ is an abelian group given by the group cohomology of $S L(2, K)$. It is a subquotient of the group $\mathbb{Z}\left[K^{\times}\right]$(the free abelian group which has one generator $[x]$ for each non-zero $x \in K)$. Thus the elements of $B(K)$ can be labelled by elements of $\mathbb{Z}\left[K^{\times}\right]$, though not in a unique way. The torsion sugroup of an abelian group is the subgroup consisting of the elements of finite order. The torsion subgroup of $\mathbb{Z}\left[K^{\times}\right]$is trivial, but in the Bloch groups relations like $[1 / 2]+[1 / 2]=0$ may introduce non-trivial torsion. We shall study a map from finite order elements to the central charges and scaling dimensions of conformal field theories. The mapping is provided by the dilogarithm, which is a function known for unexpected appearances in diverse fields in mathematics and physics, notably perturbative quantum field theory (see Weinzierl's talk W03), the classification of hyperbolic three-manifolds, and algebraic $K$-theory. In perturbation theory and algebraic $K$-theory, higher polylogarithms appear, too, but it is not known if there is a connection.

Integrable quantum field theories have been treated by the thermodynamic Bethe ansatz, and in this context the dilogarithm formulas were discovered Z91, at first for real argument. For extensions to complex arguments, see M91, N93, DT98. The original derivation of the formulas was cumbersome, but we shall study a much easier one. In particular, we do not need any thermodynamics. The argument is presented in the second half of section three. Specialists may start to read there, since the preceding part of the article concerns well known facts and is quite elementary.

Indeed, much of this article is aimed at mathematicians who want to see quantum field theory in an understandable language and for young physicists who do not want to learn it as an exclusive art. Thus the next section is a pedagogical introduction into the most elementary aspects of quantum field theory. No history of the ideas is given, all results are standard, and almost all calculations should be easily reproducible by the reader. Functional integrals are irrelevant. The language of vertex operator algebras will only be mentioned in this introduction, since I think that it is a bit far from God's book of optimal proofs and since the standard physics formulation is far better suited for the discussion of perturbations which break conformal invariance. Physically, vertex operator algebras describe the operator product expansions of holomorphic and anti-holomorphic fields, which will of course be central to the later discussions. Free fermions and the simplest minimal models will be considered in enough detail to obtain a first concrete understanding of the relations between some quantum field theories and algebraic $K$-theory. Hopefully, the newcomer who works through this material will find it helpful for the study of more complex cases. 
A rational CFT has a holomorphic and an anti-holomorphic vertex operator algebra. They have finite sets of characters $\chi_{i}, \bar{\chi}_{j}$, resp. The $\chi_{i}$ are holomorphic functions of the complex upper half plane and have the form

$$
\chi_{i}(\tau)=q^{h_{i}-c / 24} \sum_{n=0}^{\infty} a_{i n} q^{n},
$$

with $q=\exp (2 \pi i \tau)$, rational $c, h_{i}$ and positive integers $a_{i n}$. For the distinguished vacuum character $\chi_{0}$ one has $h_{0}=0, a_{00}=1$. The $q$ exponents $h_{i}+n$ are the holomorphic conformal dimensions of the CFT, and $c$ is its holomorphic central charge. The minimal value among the differences $c-24 h_{i}$ is called the effective central charge $c_{\text {eff }}$. In unitary theories $h_{i}>0$ for $i \neq 0$, such that $c_{e f f}=c$. After complex conjugation, the properties of the $\bar{\chi}_{j}$ are analogous, and in many cases these characters are indeed the complex conjugates of the $\chi_{i}$. We are interested in massive perturbations without holomorphic or anti-holomorphic fields. In CFTs with such perturbations the holomorphic and anti-holomorphic central charges have to be equal.

The partition function of a bosonic rational CFT has the form

$$
Z=\sum_{i, j} n_{i j} \chi_{i} \bar{\chi}_{j}
$$

where $n_{00}=1$ and all $n_{i j}$ are positive integers (possibly zero). As a consequence of conformal invariance, the partition function $Z$ is invariant under the modular group $S L(2, \mathbb{Z})$, the elements $\left(\begin{array}{l}A B \\ C D\end{array}\right)$ of which act on $\tau$ in the form $\tau \mapsto(A \tau+B) /(C \tau+D)$. Consequently, the $\chi_{i}$ form a vector valued representation of the modular group, such that they transform into a linear combinations of each other, with constant coeffients. Functions which generate a finite dimensional vector space under $S L(2, \mathbb{Z})$ transformations will be called modular. The terminology is not quite the standard one, but this shouldn't matter, since the individual $\chi_{i}$ are also expected to be invariant under a congruence subgroup of $S L(2, \mathbb{Z})$. We will not need this property, however. Fermionic CFTs have slightly more structure in their partition functions, but their characters are modular, too.

The property which is essential for us is the behaviour under the particular modular transformation $\tau \mapsto-1 / \tau$, namely the fact that the $\chi_{i}$ can be written in the form

$$
\chi_{i}(\tau)=\sum_{k} \tilde{a}_{i k} \tilde{q}^{k}
$$

with $\tilde{q}=\exp (-2 \pi i / \tau)$, where the sum goes over rational numbers of the form $k=h_{j}-c / 24+n, n \in \mathbb{N}$. For small $\tau$ the dominating exponent is $k_{0}=-c_{e f f} / 24$, and the corresponding coefficient $\tilde{a}_{i k_{0}}$ is real and strictly positive for all $i$. All coefficients $\tilde{a}_{i k}$ turn out to be algebraic numbers, but they will not be studied in this article.

Apart from the factor $q^{h_{i}-c / 24}$ the $\chi_{i}$ also can be described in a combinatorial way, sometimes in terms of very classical combinatorics. We shall see in 
the next sections how this comes about. For the moment let us just list some of the relevant functions. The partitions of natural numbers into $n$ distinct summands have the generating function

$$
\frac{q^{\left(n^{2}+n\right) / 2}}{(q)_{n}}
$$

where $(q)_{n}=(1-q)\left(1-q^{2}\right) \cdots\left(1-q^{n}\right)$. Thus

$$
\sum_{n \in \mathbb{N}} \frac{q^{\left(n^{2}+n\right) / 2}}{(q)_{n}}=\prod_{n=1}^{\infty}\left(1+q^{n}\right)
$$

(in our dialect, $\mathbb{N}=\{0,1, \ldots\}$ ). Analogously, the partitions into distinct odd summands yield

$$
\sum_{n \in \mathbb{N}} \frac{q^{n^{2}}}{\left(q^{2}\right)_{n}}=\prod_{n=1}^{\infty}\left(1+q^{2 n+1}\right) .
$$

Up to factors $q^{1 / 24}$ and $q^{-1 / 24}$, resp., these are modular functions. Another example of equalities between such sums and products is provided by the Rogers-Ramanujan identities

$$
\begin{aligned}
\sum_{n \in \mathbb{N}} \frac{q^{n^{2}+n}}{(q)_{n}} & =\prod_{n \equiv 2,3 \bmod 5}\left(1-q^{n}\right)^{-1} \\
\sum_{n \in \mathbb{N}} \frac{q^{n^{2}}}{(q)_{n}} & =\prod_{n \equiv 1,4 \bmod 5}\left(1-q^{n}\right)^{-1} .
\end{aligned}
$$

Again these are modular functions, up to factors $q^{11 / 60}$ and $q^{-1 / 60}$, resp. A first generalization is provided by the Andrews-Gordon identities A76.

A more surprising example concerns the partitions of the natural numbers into distinct half-integral summands, for which one obtains KM93.

$$
\sum_{n \in \mathbb{N}} \frac{q^{2 n^{2}}}{(q)_{2 n}}=\sum_{m \in \mathbb{N}^{8}} \frac{q^{m C m}}{(q)_{m}} .
$$

Here $m=\left(m_{1}, \ldots, m_{8}\right),(q)_{m}=(q)_{m_{1}} \cdots(q)_{m_{8}}$, and

$$
C=\left(\begin{array}{cccccccc}
2 & 3 & 4 & 5 & 6 & 4 & 3 & 2 \\
3 & 6 & 8 & 10 & 12 & 8 & 6 & 4 \\
4 & 8 & 12 & 15 & 18 & 12 & 9 & 6 \\
5 & 10 & 15 & 20 & 24 & 16 & 12 & 8 \\
6 & 12 & 18 & 24 & 30 & 20 & 15 & 10 \\
4 & 8 & 12 & 16 & 20 & 14 & 10 & 7 \\
3 & 6 & 9 & 12 & 15 & 10 & 8 & 5 \\
2 & 4 & 6 & 8 & 10 & 7 & 5 & 4
\end{array}\right)
$$


is the inverse of the Cartan matrix of the exceptional Lie algebra $E_{8}$. Formula (5) is still unproven, but easy to check to high order. Much work has been done on the combinatorial side, see e.g. [BM98, ABD03]. The present article can only give a few hints in this direction.

The combinatorial structures have deep roots in quantum field theory, In section 3 we shall obtain the form

$$
\chi_{i}(\tau)=\sum_{m} \frac{q^{Q_{i}(m)}}{(q)_{m}}
$$

with

$$
Q_{i}(m)=m A m / 2+b_{i} m+h_{i}-c / 24
$$

for charcters of certain CFTs with integrable deformations. Note that the matrix $A$ is the same for all characters $\chi_{i}$ of a given CFT. The form comes from the Bethe ansatz, which is a well tested conjecture concerning the solution of integrable theories. The derivation is very simple and straightforward, but seems to be new.

In section 4 we consider general sums of the form $\sum_{m} q^{Q(m)} /(q)_{m}$, where $Q(m)=m A m / 2+b m+h$ has rational coefficients. We shall see that such a function can only be modular when all solutions of the system of equations

$$
\sum_{j} A_{i j} \log \left(x_{j}\right)=\log \left(1-x_{i}\right)
$$

yield elements $\sum_{i}\left[x_{i}\right]$ of finite order in the Bloch group of the algebraic numbers, or more precisely in a certain extension of it which takes into account the multivaluedness of the logarithm. The argument is incomplete, but it should not be too difficult to make it rigorous.

Note that exponentiation of eq. (17) yields algebraic equations for $x_{i}$, with a finite number of solutions. In important special cases the $x_{i}$ are real and positive. In this case the multivaluedness of the logarithm is irrelevant and one can work with the Bloch group $B\left(\overline{\mathbb{Q}}^{+}\right)$of the field $\overline{\mathbb{Q}}^{+}$of all real algebraic numbers or equivalently with $B(\mathbb{R})=B\left(\overline{\mathbb{Q}}^{+}\right)$. This group has a torsion subgroup isomorphic to $\mathbb{Q} / \mathbb{Z}$, which naturally encodes conformal dimensions $h_{i}$ modulo the integers. It is not really necessary to consider the fields $\mathbb{R}$ and $\mathbb{C}$ instead of their algebraic subfields, but for physicists it certainly is natural.

In general it may seem natural to work with the Bloch group $B(\mathbb{C})=B(\overline{\mathbb{Q}})$, where $\overline{\mathbb{Q}}$ is the field of all algebraic numbers, but here it is inappropriate to forget about the multivaluedness of the logarithm, since the torsion subgroup of $B(\mathbb{C})$ is trivial $\mathbf{S 8 6}$, S89. Our quantum field theory context leads to an extension $\hat{B}(\mathbb{C})$ of $B(\mathbb{C})$, however, in which $\mathbb{C}$ is replaced by a cover $\hat{\mathbb{C}}$ of $\mathbb{C}-\{0,1\}$ on which $\log (x)$ and $\log (1-x)$ are single-valued. The usual Bloch group $B(\mathbb{C})$ is just the quotient of $\hat{B}(\mathbb{C})$ by its torsion subgroup, which again 
is isomorphic to $\mathbb{Q} / \mathbb{Z}$. The group $\hat{B}(\mathbb{C})$ is a very natural object in algebraic $\mathrm{K}$ theory and emerged in the study of hyperbolic three-manifolds in the context of Thurston's program [NZ85, GZ00, N03.

In Thurston's program, hyperbolic three-manifolds are described by triangulations into tetrahedra in the standard hyperbolic space $\mathbb{H}^{3}$. Every triangulation represents an element of $\hat{B}(\mathbb{C})$, and a change of the triangulation gives another $\mathbb{Z}[\hat{\mathbb{C}}]$ representation of the same element. Moreover, there is a map $D: B(\mathbb{C}) \rightarrow \mathbb{R}$ which yields the volume of the manifold when the curvature of $\mathbb{H}^{3}$ is normalized to -1 , and this volume is an invariant of the three-manifold. Now $D$ is essentially the imaginary part of the Rogers dilogarithm $L$, which provides a natural group homomorphism $L: \hat{B}(\mathbb{C}) \rightarrow \mathbb{C} / \mathbb{Z}(2)$, as discussed in Zagier's talk. The real part of $L$ yields the Chern-Simons invariant of the manifold. The symbol $\mathbb{Z}(N)$ stands for $(2 \pi i)^{N} \mathbb{Z}$ and indicates that the right context for both the classification of three-manifolds and of our CFTs should be the theory of motives.

In the quantum field theoretical situation, we deal with the torsion subgroup of $\hat{B}(\mathbb{C})$, for which $(2 \pi i)^{2} L$ takes values in $\mathbb{Q} / \mathbb{Z}$. These values yield the conformal dimensions of the fields of the theory (more precisely the exponents $\left.h_{i}-c / 24\right)$. The fact that they are only obtained modulo the integers is natural, since each $\chi_{i}$ yields a whole family $h_{i}+n$ of conformal dimensions. Nevertheless, $h_{i} \in \mathbb{Q}$ is the smallest one among them, and a refinement of the K-theory should yield this number. Similarly, in the study of three-manifolds it should be possible to remove the ambiguity of the Chern-Simons invariant.

The geometrical ideas just mentioned have been used to derive dilogarithm identities in conformal field theory [GT96, but the construction of a more direct link between Thurston's program and conformal field theory may be another task for the future. It is tempting to relate the conformal field theories in two dimensions to topological ones in three dimensions and to use the latter for calculating invariants of the three-manifolds.

Here we will be concerned by more elementary issues, however. In section 5 we will take a closer look at eqs. (17). One knows many matrices $A$ for which these equations yield torsion elements of the extended Bloch group. The best known examples are related to Cartan matrices, or equivalently to Dynkin diagrams. For Dynkin diagrams of A type, we will find all solutions of the equations, whereas so far only one solution was known K87, KR87, up to the action of the Galois group. It turns out that $x_{i}$ are rational linear combinations of roots of unity, and we expect that this holds whenever eq. (7) yields Bloch group elements of finite order.

For each Dynkin diagram of ADET type, the analysis of eq. (7) uses a family of polynomials which are linked by linear and quadratic recursion relations. For $\mathrm{A}_{1}$ one finds the Chebysheff polynomials. To study the general case one needs the representation theory of simple Lie groups and their quantum group deformations. Relations to quivers and Ocneanu's essential paths on the Dynkin diagrams 099 are likely, but will not be explored here. Many different mathematical themes appear, from the elementary ones central to this article 
up to rather advanced issues. The explanations will take the mathematical reader straight into quantum field theory and the physicist into some interesting areas of algebra. For both, parts of the article will be too well known to merit much attention, but when one addresses a mixed audience this is unavoidable. More serious is the fact that most of the obvious questions will remain open - the end of this article is dictated by the present status of research and of my understanding, not by any intrinsic logic. I hope that some readers will do better.

\section{Acknowledgments}

I wish to thank Edward Frenkel, Herbert Gangl and Don Zagier for extensive and helpful discussions which were essential for this article.

\section{Free and conformally invariant quantum field theories}

The only quantum field theories which are easily understood from a mathematical point of view are the free ones and (some of) the conformally invariant ones. The intersection of these two families yields the theory of massless fermions in two dimensions, which we will study as our basic example. Mathematically this CFT is very simple, but it allows to understand many general features of quantum field theory, in particular the operator product expansion. The theory of free massive fermions can be considered as a perturbation, and we will use it to consider basic features of perturbation theory.

We first show how to quantize arbitrary theories of free fermions. Aspects of conformal invariance will be introduced later. Free means linear, and all one needs is a slight generalization of the quantization of the harmonic oscillator. Recall that the phase space $V$ of a classical harmonic oscillator is a finite dimensional vector space with a non-degenerate anti-symmetric bilinear form. For definiteness one should consider a two-dimensional $V$ with coordinates $x, p$ and a bilinear form obtained from $\{x, p\}=1$, but the step to quantum field theory is easier when one uses a more abstract language.

The abelian group $V$ acts on the phase space (on itself) by translation, and one still wants to have such an action on a space of states after quantization. Thus one constructs a Hilbert space $H$ on which $V$ is projectively represented. More precisely, $H$ carries a representation of an extension of $V$ by the complex numbers of modulus one, and this extension is given by the bilinear form.

In a more physical way the same procedure can be described as follows. The linear functions on $V$ are observables of the classical theory. One wants them to become observables of the quantized theory, too. The commutators of these observables yield the Heisenberg Lie algebra which is given by the bilinear form, up to a factor of $i$. More precisely the vector space of this Lie algebra is $\mathbb{C} \oplus V_{\mathbb{C}}^{*}$, where $V^{*}$ is the dual of $V$ and $V_{\mathbb{C}}^{*}$ its complexification. This is just the infinitesimal version of the previous description, since the 
linear functions on $V$ generate translations in the Hamilton formalism and the non-degenerate bilinear form yields a natural isomorphism between $V$ and $V^{*}$. In the standard two-dimensional example the observables satisfy the Heisenberg Lie algebra given by $[x, p]=i$, translations of $x$ by $a$ are given by $\exp (-i a p)$ and translations of $p$ by $\exp (i a x)$. The extra $i$ in $[x, p]=i$ compared to $\{x, p\}=1$ is necessary, since hermitean $x, p$ yield anti-hermitean $[x, p]$. In the fermionic case it is unnecessary, which saves us some trouble.

For fermionic theories nothing much changes, the vector space $V$ just has a symmetric bilinear from instead of an anti-symmetric one. Thus one works with a super-Lie analogue of the Heisenberg algebra. Moreover only the even polynomials in $V$ correspond to observables, so one has to introduce a $\mathbb{Z}_{2}$ grading.

Now we can start to construct the simplest quantum field theory. We will need some care and elementary distribution theory to handle an infinite dimensional $V$, but the main steps are just as before. Let $V$ be a real vector space with non-degenerate symmetric bilinear form $\{$,$\} . We regard V$ as the odd part of a $\mathbb{Z}_{2}$-graded vector space $\mathbb{R} \oplus V$. The form defines a super-Lie algebra on $\mathbb{R} \oplus V$, with center $\mathbb{R}$, and also on the complexification $\mathbb{C} \oplus V_{\mathbb{C}}$ of $\mathbb{R} \oplus V$. We are interested in representations of this super-Lie algebra for which $1 \in \mathbb{R}$ is represented by the identity.

Let $V_{\mathbb{C}}=V_{+} \oplus V_{-}$be a decomposition of the complexification of $V$ into isotropic, complex conjugate subspaces. Then we have a natural super-Lie algebra representation $\hat{\psi}: V_{\mathbb{C}} \rightarrow \operatorname{End}(H)$ with

$$
H=\Lambda V_{-}=\oplus_{n} \Lambda^{n} V_{-},
$$

such that $\hat{\psi}\left(v_{+}\right)$annihilates $\Lambda^{0} V_{-}=\mathbb{C}$ for $v_{+} \in V_{+}$, whereas for $v_{-} \in V_{-}$, $w \in H$ one has

$$
\hat{\psi}\left(v_{-}\right) w=v_{-} \wedge w .
$$

One finds that $V_{+}$acts as a super-derivation, induced by

$$
\hat{\psi}\left(v_{+}\right) v_{-}=\left\{v_{+}, v_{-}\right\} .
$$

The induced hermitean from on $H$ is not necessarily positive definite, but in our context we do not need $H$ to be a Hilbert space, since only direct sums of finite dimensional spaces will occur. Theories with a positive definite form are called unitary.

Let $\mathbb{R} \oplus V^{1}, \mathbb{R} \oplus V^{2}$ be two superalgebras of the type just discussed, with representations on $H^{i}, i=1,2$. When $V=V^{1} \oplus V^{2}$ and $\left\{v^{1}, v^{2}\right\}=0$ for $v^{i} \in$ $V^{i}$, then $\mathbb{R} \oplus V$ has a natural representation on $H^{1} \otimes H^{2}$. For representations obtained from isotropic subspaces $V_{-}^{i}$ there is indeed a natural isomorphism $\Lambda\left(V_{-}^{1} \oplus V_{-}^{2}\right) \simeq \Lambda V_{-}^{1} \otimes \Lambda V_{-}^{1}$.

The enveloping algebra of $\mathbb{R} \oplus V$ is the Clifford algebra given by $V$ and $\{$,$\} .$ When the dimension of $V$ is finite, our construction demands that it is even, since $\operatorname{dim}(V)=2 \operatorname{dim}\left(V_{+}\right)$. In this case it is well known that all irreducible 
representations are isomorphic. When $\operatorname{dim}(V)$ is odd, recall that we want a $\mathbb{Z}_{2}$ graded representation. Let the $\mathbb{Z}_{2}$ grading of $H$ be given by $H=H_{b} \oplus H_{f}$ with an operator $\mathcal{F}$ which acts as +1 on $H_{b}$ and as -1 on $H_{f}$. Thus we want a representation of $\mathbb{R} \oplus V \oplus\langle\mathcal{F}\rangle$, which brings us back to the even case.

But let us return to $V_{\mathbb{C}}=V_{+} \oplus V_{-}$. Let $1^{*} \in H^{*}$ be the projection to $\Lambda^{0} V_{-}=\mathbb{C}$. The vectors $1 \in H$ and $1^{*} \in H^{*}$ are called vacuum vectors, and the vacuum expectation value of an operator $O \in \operatorname{End}(H)$ is denoted by $\langle O\rangle=1^{*} O 1$. For odd $n$ and $v_{i} \in V$ we have $\left\langle\hat{\psi}\left(v_{1}\right) \hat{\psi}\left(v_{2}\right) \ldots \hat{\psi}\left(v_{n}\right)\right\rangle=0$, since $\hat{\psi}\left(v_{i}\right) \Lambda^{n} V_{-} \subset \Lambda^{n-1} V_{-} \oplus \Lambda^{n+1} V_{-}$. For even $n$ we have

$$
\left\langle\hat{\psi}\left(v_{1}\right) \hat{\psi}\left(v_{2}\right) \ldots \hat{\psi}\left(v_{n}\right)\right\rangle=\operatorname{Pf}(M),
$$

where $P f$ is the Pfaffian and the anti-symmetric $n \times n$ matrix $M$ has entries $M_{i j}=\left\langle\hat{\psi}\left(v_{i}\right) \hat{\psi}\left(v_{j}\right)\right\rangle$ for $i \neq j$ (Wick's thorem). We have

$$
\left\langle\hat{\psi}\left(v_{i}\right) \hat{\psi}\left(v_{j}\right)\right\rangle=\left\{v_{i} v_{j}^{-}\right\},
$$

where $v_{j}^{-}$is the projection of $v_{j}$ to $V^{-}$.

For the harmonic oscillator, phase space can be considered as the space of initial conditions for the equations of motion, or more invariantly as the space of solutions of these equations. The latter are linear ordinary differential equations. In free quantum field theory, $V$ is the solution space of some linear partial differential equations. We shall work in two dimensions, with time coordinate $t \in \mathbb{R}$ and space coordinate $x$. Often one considers $x \in \mathbb{R}$, but we shall concentrate on the case of the unit circle $S^{1}$ with angle $x$.

We study the two-dimensional Dirac equation for a real two-component spinor $\psi=\left(\psi_{R}, \psi_{L}\right)$,

$$
\begin{aligned}
& \left(\partial_{x}-\partial_{t}\right) \psi_{R}(x, t)=\mu \psi_{L}(x, t) \\
& \left(\partial_{x}+\partial_{t}\right) \psi_{L}(x, t)=\mu \psi_{R}(x, t),
\end{aligned}
$$

with the symmetric bilinear form

$$
\left(\psi^{1}, \psi^{2}\right)=\int_{t=t_{0}}\left(\psi_{R}^{1} \psi_{R}^{2}+\psi_{L}^{1} \psi_{L}^{2}\right) d x .
$$

Note that this form is independent of the choice of $t_{0}$. When the mass $\mu$ is different from zero it introduces a length scale, but for $\mu=0$ the equations are invariant under the conformal transformations

$$
(x-t, x+t) \mapsto(f(x-t), g(x+t)),
$$

where $f, g$ are orientation preserving diffeomorphisms of $S^{1}$. Later we will come back to the case $\mu \neq 0$, but in this section we will consider the conformally invariant theory.

For $\mu=0$ the equations for $\psi_{R}$ and $\psi_{L}$ decouple and can be treated separately. Moreover, they can be solved trivially in terms of the value of $\psi$ 
at fixed time. Thus we can forget about differential equations and regard the case where $V$ is the vector space of square integrable functions on $S^{1}$, with the standard bilinear form given by the measure $d x$. The spectrum of the rotation generator allows to decompose $V_{\mathbb{C}}$ into a positive part $V_{+}$spanned by $\exp (i m x)$ with $m>0$, a negative part $V_{-}$with $m<0$, and the constants, or zero modes, which form a one-dimensional vector space $V_{0}$. This is the R case considered by Ramond. Since the bilinear form is invariant under rotations, $V_{+}$ and $V_{-}$are isotropic. The constants are orthogonal to $V_{+}, V_{-}$, such that $\mathbb{R} \oplus V$ has representations on $\Lambda^{n} V_{-} \otimes H_{0}$, where $H_{0}$ is the standard two-dimensional representation space of the quaternion algebra given by $\mathbb{R} \oplus V_{0} \oplus\langle\mathcal{F}\rangle$. A basis of $\Lambda^{n} V_{-}$or equivalently of $\Lambda^{n} V_{+}$corresponds to the partitions of natural numbers into $n$ distinct summands $m>0$, which yields one of the partition functions mentioned in the introduction.

The complications with the zero modes can be avoided when one considers the anti-periodic functions on the double cover of $S^{1}$ instead, in other words the sections of the Möbius bundle. This is the NS case considered by Neveu and Schwarz. All fermionic CFTs have NS and R sectors, so it is useful to introduce them together. In the NS case, $V_{+}$is spanned by $\exp (i r x)$, where $r=1 / 2,3 / 2, \ldots$. A basis of $\Lambda^{n} V_{+}$corresponds to the partitions of natural numbers into $n$ distinct odd summands $2 r$.

The NS case arises naturally when we take into account conformal invariance. The scalar product $\int f(x) g(x) d x$ of two functions is no longer natural, since the differential $d x$ on $S^{1}$ changes under diffeomorphisms. Instead we have to factorize

$$
\psi^{1}(x) \psi^{2}(x) d x=\left(\psi^{1}(x) \sqrt{d x}\right)\left(\psi^{2}(x) \sqrt{d x}\right) .
$$

Thus $V$ should be regarded as a vector space of half-differentials, in other words of sections of a squareroot of the cotangent bundle. Two different squareroots exist, which correspond to the NS and R cases. Considering $S^{1}$ as the boundary of the complex unit disc we write $z=\exp (i x)$. The cotangent bundle on the disc has a unique squareroot with a section $\sqrt{d z}$. On the boundary it reduces to the complexification of the Moebius bundle with section $\sqrt{d z}=\exp (i x / 2) \sqrt{d x}$. Since $\exp (i x / 2)$ is anti-periodic, the NS case is in a sense more basic than the $\mathrm{R}$ case.

Anticipating some future simplifications we write

$$
\hat{\psi} \sqrt{2 \pi i d x}=\psi \sqrt{d z} .
$$

This is just a change of normalization by $\exp (i x / 2) / \sqrt{2 \pi}$.

The mathematical reader may wonder what all of this has to do with quantum field theory, since it is just very conventional mathematics, but now comes the essential step. We can consider $\psi$ as an operator valued distribution, which to a function $v$ on $S^{1}$ associates the operator $\psi(v)$ on $H$. Note that $\psi(v) \psi\left(v^{\prime}\right)=-\psi\left(v^{\prime}\right) \psi(v)$ when the supports of $v, v^{\prime}$ have empty intersection. In physics terminology, such operator valued distributions are called fermionic 
local fields on $S^{1}$. In general a local field theory on $S^{1}$ provides a $\mathbb{Z}_{2}$-graded vector space of fields $F=F_{b} \oplus F_{f}$, with degree $\eta(\phi)=0$ for $\phi \in F_{b}$ and $\eta(\phi)=1$ for $\phi \in F_{f}$ such that

$$
\phi(v) \chi\left(v^{\prime}\right)=(-)^{\eta(\phi) \eta(\chi)} \chi\left(v^{\prime}\right) \phi(v)
$$

when the test functions $v, v^{\prime}: S^{1} \rightarrow \mathbb{C}$ have non-intersecting support. The space $F_{b}$ contains the bosonic fields and $F_{f}$ the fermionic ones.

In this section we only need fields on $S^{1}$, but let us indicate what happens in more general contexts. The functions $v, v^{\prime}$ become test functions on a spacetime with causal structure and the equation $\phi(v) \chi\left(v^{\prime}\right)= \pm \chi\left(v^{\prime}\right) \phi(v)$ applies, with appropriate sign, when the supports of $v, v^{\prime}$ are causally independent.

The simple case we consider is called the quantum field theory of a free fermion and the distribution $\psi$ is a free fermion field. The vector $1 \in H$ is called the vacuum, and the vacuum expectation value $\left\langle\psi\left(v_{1}\right) \psi\left(v_{2}\right) \ldots \psi\left(v_{n}\right)\right\rangle$ can be considered as the value of a distribution on the $n$-fold Cartesian product of the circle, evaluated at $v_{1} \otimes \ldots \otimes v_{n}$. This distribution is written $\langle\psi \ldots \psi\rangle$. By Wick's theorem (8), it is sufficient to calculate $\langle\psi \psi\rangle$. By eq. (9) we have

$$
\left\langle\hat{\psi}(v) \hat{\psi}\left(v^{\prime}\right)\right\rangle=\int v(x) v_{-}^{\prime}(x) d x
$$

where

$$
v_{-}^{\prime}(x)=\sum_{r>0} \exp (-i r x) \int v^{\prime}(y) \exp (\text { iry }) \frac{d y}{2 \pi} .
$$

Thus the distribution $\langle\hat{\psi} \hat{\psi}\rangle$ is the $\epsilon \rightarrow+0$ limit of the function

$$
\frac{1}{2 \pi} \sum_{r>0} \exp (-i r x) \exp (\text { iry })=\frac{1}{2 \pi} \exp (i x / 2) \exp (i y / 2) \frac{1}{\exp (i x)-\exp (i y)}
$$

where $\Im x=0, \Im y=\epsilon$, such that the absolute value of $\exp (i y)$ approaches the one of $\exp (i x)$ from below. In terms of $\psi=\sqrt{2 \pi} \exp (-i x / 2) \hat{\psi}$, we see that the distribution $\langle\psi \psi\rangle$ is the limiting value of the function

$$
\langle\psi \psi\rangle(z, w)=(z-w)^{-1}
$$

where $z=\exp (i x), w=\exp (i y)$, and $|z|$ approaches $|w|$ from above. More generally, for even $n$ the distribution $\langle\psi \ldots \psi\rangle$ on the $n$-fold Cartesian product of the circle is a limiting value of a function on $\mathbb{C}^{n}$ given by

$$
\langle\psi \ldots \psi\rangle\left(z_{1}, \ldots, z_{n}\right)=\operatorname{Pf}(M)
$$

with $M_{i j}=\left(z_{i}-z_{j}\right)^{-1}$ off the diagonal. Such functions are called $n$-point functions. By convention, they are written in the form $\left\langle\psi\left(z_{1}\right) \ldots \psi\left(z_{n}\right)\right\rangle$. In the distributional limit the $\left|z_{k}\right|$ all tend to 1 and the limit is taken along a path such that $\left|z_{i}\right|>\left|z_{j}\right|$ for $i<j$. The only singularities of the $n$-point 
function occur at the partial diagonals $z_{i}=z_{j}$. For fermionic fields, as in the present case, the $n$-point functions are anti-symmetric in all variables, which we express in the form $\psi(z) \psi(w)=-\psi(w) \psi(z)$.

In general, quantum field theories can be formulated either in terms of operator valued distributions or of $n$-point functions. We mainly will use the latter formulation, which is called euclidean quantum field theory. So far we considered a single field $\psi$, but in general one needs bosonic and fermionic fields in a vector space $F=F_{b} \oplus F_{f}$. A rather trivial but important element is the identity field $I \in F_{b}$ which does not depend on $z$ and satisfies

$$
\left\langle I \phi^{1}\left(z_{1}\right) \ldots \phi^{n}\left(z_{n}\right)\right\rangle=\left\langle\phi^{1}\left(z_{1}\right) \ldots \phi^{n}\left(z_{n}\right)\right\rangle
$$

for all $\phi^{k} \in F$. Let $T(F)=\oplus_{n} T^{n}(F)$ be the tensor algebra over $F$. Then the $n$-point functions map $T^{n}(F)$ to the space of functions on $\mathbb{C}^{n}$ minus the partial diagonals, and the map factors through the projection from $T(F)$ to $S F_{b} \otimes \Lambda F_{f}$.

For $N$ quantum field theories with field spaces $F^{k}, k=1, \ldots, N$, one has a natural tensor product with field space $\otimes F^{k}$. The $n$-point functions of the product theory are the products of the $n$-point functions of the factors. There is a natural embedding $F^{i} \rightarrow \otimes F^{k}, i=1, \ldots, N$, since one can identify $I \otimes \phi$ with $\phi$. For $N$ free fermion theories the product has fields $\psi^{k}, k=1, \ldots, N$, with $n$-point functions

$$
\left\langle\psi^{k_{1}}\left(z_{1}\right) \ldots \psi^{k_{n}}\left(z_{n}\right)\right\rangle=\operatorname{Pf}(M),
$$

for even $n$, where now

$$
M_{i j}=\delta_{k_{i}, k_{j}}\left(z_{i}-z_{j}\right)^{-1} .
$$

Equivalently we could generalize our simplest example such that $V$ is given by maps from $S^{1}$ to $\mathbb{R}^{N}$, with standard bilinear form. Then every element $a \in \mathbb{R}^{N}$ yields one local quantum field $\psi_{a} \in F_{f}$ which maps functions $f: S^{1} \rightarrow \mathbb{R}$ to $\psi_{a}(f)=\psi(f a)$. The $\psi^{k}$ the correspond to the standard basis of $\mathbb{R}^{N}$.

We abstract certain features from our examples, which will be valid in general. There is a vector $1 \in H$ and a vector $1^{*}$ in its dual such that the $n$ point functions $\left\langle\phi^{1}\left(z_{1}\right) \ldots \phi^{n}\left(z_{n}\right)\right\rangle=1^{*} \phi^{1}\left(z_{1}\right) \ldots \phi^{n}\left(z_{n}\right) 1$ are translationally invariant,

$$
\left\langle\phi^{1}\left(z_{1}+z_{0}\right) \ldots \phi^{n}\left(z_{n}+z_{0}\right)\right\rangle=\left\langle\phi^{1}\left(z_{1}\right) \ldots \phi^{n}\left(z_{n}\right)\right\rangle .
$$

These functions are real analytic away from the partial diagonals. When all $n$-point functions involving $\phi \in F$ vanish, then $\phi=0$. Thus fields can be determined by their $n$-point functions.

A field $\phi$ is called holomorphic when all $n$-point functions $\langle\phi(z) \ldots\rangle$ depend meromorphically on $z$, as in our basic example. For each pair of fields $\phi, \phi^{1}$ with holomorphic $\phi$ we construct a family of fields $N_{m}\left(\phi, \phi^{1}\right)$ whose $n$ point functions are given by the Laurent expansion of $(n+1)$-point functions involving $\phi, \phi^{1}$, i.e. 


$$
\left\langle\phi(z) \phi^{1}\left(z_{1}\right) \ldots \phi^{n}\left(z_{n}\right)\right\rangle=\sum_{m}\left(z-z_{1}\right)^{m}\left\langle N_{m}\left(\phi, \phi^{1}\right)\left(z_{1}\right) \phi^{2}\left(z_{2}\right) \ldots \phi^{n}\left(z_{n}\right)\right\rangle
$$

in the sense of a Laurent expansion in $z$ around the possible pole at $z=$ $z_{1}$. Note that this equation is assumed to be valid for all choices of the $\phi^{k}$. Symbolically it is written in the form

$$
\phi(z) \phi^{1}(w)=\sum_{m}(z-w)^{m} N_{m}\left(\phi, \phi^{1}\right)(w) .
$$

This is the simplest case of the operator product expansion (OPE) of local fields. The field $N_{0}\left(\phi, \phi^{1}\right)$ is called the normal ordered product of $\phi, \phi^{1}$. The OPE also can be defined when $\phi$ is not holomorphic, but then the functions $(z-w)^{m}$ are replaced by non-universal sets of real analytic functions.

The OPE is compatible with the $\mathbb{Z}_{2}$-grading. In particular, for $\phi, \phi^{1} \in F_{f}$ one has $N_{m}\left(\phi, \phi^{1}\right) \in F_{b}$ for all $m$. For the free fermion, $N_{-1}(\psi, \psi)=I$.

In our basic example the $n$-point functions are homogeneous with respect to linear transformations of $\mathbb{C}$. More generally, conformal invariance yields a grading $h$ of the vector space of holomorphic fields, such that their $n$-point functions satisfy

$$
\left\langle\phi^{1}\left(a z_{1}\right) \ldots \phi^{n}\left(a z_{n}\right)\right\rangle=a^{-h_{\Sigma}}\left\langle\phi^{1}\left(z_{1}\right) \ldots \phi^{n}\left(z_{n}\right)\right\rangle
$$

where

$$
h_{\Sigma}=\sum_{k=1}^{n} h\left(\phi^{k}\right)
$$

One easily sees that

$$
h\left(N_{m}\left(\phi^{1}, \phi^{2}\right)\right)=m+h\left(\phi^{1}\right)+h\left(\phi^{2}\right) .
$$

In particular, $h(I)=0$, and $h(\psi)=1 / 2$ for free fermion fields. More generally, bosonic holomorphic fields have integral and fermionic ones half-integral conformal dimension. When one lets $a$ vary over the unit circle, one sees thate $n$-point functions involving an odd number of fermionic fields have to vanish.

Now we can define holomorphic field theories. There is a vector space $F$ with a grading $F=\sum_{h \in \mathbb{N} / 2} F(h)$ such that $F(0)=\mathbb{C}$. Let $\oplus_{h \in \mathbb{N}} F(h)=F_{b}$ and $\oplus_{h \in \mathbb{N}+1 / 2} F(h)=F_{f}$. There is an $n$-point function map from $S F_{b} \otimes \Lambda F_{f}$ to the meromorphic functions which are holomorphic away from the partial diagonals, such that these functions are translationally invariant and satisfy the scaling relation (13). With respect to these functions, $F$ must be closed under the OPE. No field must have identically vanishing $n$-point functions. Finally, for sufficently large $N$ and all $h$

$$
\operatorname{dim} F(h) \leq \operatorname{dim} F^{N}(h),
$$

where $F^{N}$ is the field space of the theory of $N$ free fermions. 
A large class of such theories can be constructed by taking the OPE closure of some subspace of $F^{N}$ and factoring out those fields which have identically vanishing $n$-point functions. One example is $F_{b}^{N}$, but we will consider several others. Some further constructions will be considered below.

Scale invariant non-holomorphic theories can be defined in the same way. First let us construct examples. Take a holomorphic theory with field space $F_{h o l}$, and the complex conjugate of the $n$-point functions. This is a theory of anti-holomorphic fields, with a field space $F_{\overline{h o l}}$ anti-linearly isomorphic to $F_{h o l}$. The tensor product of the two theories has a field space $F_{h o l} \otimes F_{\overline{h o l}}$. There are subspaces $F_{h o l}$ and $F_{\overline{h o l}}$, but all the remaining fields are neither holomorphic nor anti-holomorphic.

Let us generalize this example. There must be a double grading $(h, \bar{h})$ of $F$, such that

$$
\left\langle\phi^{1}\left(a z_{1}\right) \ldots \phi^{n}\left(a z_{n}\right)\right\rangle=a^{-h_{\Sigma}} \bar{a}^{-\bar{h}_{\Sigma}}\left\langle\phi^{1}\left(z_{1}\right) \ldots \phi^{n}\left(z_{n}\right)\right\rangle,
$$

where

$$
\begin{aligned}
& h_{\Sigma}=\sum_{k=1}^{n} h\left(\phi^{k}\right) \\
& \bar{h}_{\Sigma}=\sum_{k=1}^{n} \bar{h}\left(\phi^{k}\right) .
\end{aligned}
$$

The sum $h(\phi)+\bar{h}(\phi)$ is called the scaling dimension of $\phi$, and $h(\phi)-\bar{h}(\phi)$ is called its conformal spin. For holomorphic $\phi$ one has $\bar{h}(\phi)=0$. When we rewrite

$$
a^{h_{\Sigma}} \bar{a}^{\bar{h}_{\Sigma}}=|a|^{h_{\Sigma}+\bar{h}_{\Sigma}}(a /|a|)^{h_{\Sigma}-\bar{h}_{\Sigma}}
$$

in eq. (15), we see that one can admit arbitrary real scaling dimensions, as long as the conformal spins are integral for bosonic fields and half-integral for fermionic ones. For non-holomorphic fields, the conformal dimensions $(h, \bar{h})$ may be negative. One even could admit complex scaling dimensions, but we will avoid that.

One demands that the $n$-point functions are real analytic away from the the partial diagonals, and that $F$ is closed under the OPE. The latter is slightly more complicated to define than in the holomorphic case. One best works with function germs, but we will not need the details. To control the growth of the dimensions, one works with the filtered subspaces $\oplus_{h+\bar{h}<d} F(h, \bar{h})$ and demands that for some $N$ and suffiently large $d$ the dimension of these spaces is smaller than for the theory $F^{N} \otimes \bar{F}^{N}$ of $N$ holomorphic and $N$ antiholomorphic free fermions.

In many cases, $F$ has a real structure such that the $n$-point functions for real fields at real $z^{i}$ take real values. For example, our free fermion fields $\psi$ are real. The complex conjugate theory of such a theory has a real structure, too. Often the anti-holomorphic free fermion field is called $\bar{\psi}$. This may be 
confusing, since both fields are real and linearly independent. The OPE of real fields yields real fields.

Using the OPE to deduce properties of the $n$-point functions from those of the 2-point function, one can read off from the scaling behaviour of the latter that for $z \rightarrow \infty$

$$
|\langle\phi(z) \ldots\rangle|=O\left(|z|^{-h(\phi)-\bar{h}(\phi)-\tilde{d}(\phi)}\right)
$$

where $\tilde{d}(\phi)$ is the minimal scaling dimension $h(\tilde{\phi})+\bar{h}(\tilde{\phi})$ of all fields $\tilde{\phi}$ for which $\langle\phi \tilde{\phi}\rangle \neq 0$. Thus $n$-point fuctions have good behaviour at infinity.

Consider a holomorphic field theory for which $F$ is the OPE closure of $F(1)$. Let $F(1)$ be spanned by the fields $J_{a}, a$ in some finite index set. Their OPE must have the form

$$
J_{a}(z) J_{b}(w)=\frac{d_{a b}}{(z-w)^{2}}+\sum_{c} \frac{f_{a b c}}{z-w} J_{c}(w)+\quad \text { regular terms. }
$$

Given the structure constants $d_{a b}$ and $f_{a b c}$, this is sufficient to calculate all $n$ point functions of the theory, since by induction one knows all singular terms of these meromorphic functions, and also their behaviour at infinity. Using the OPE for the four-point functions in the various possible ways, one sees that the $f_{a b c}$ must be the structure constants of a Lie algebra, and $d_{a b}$ must yield a non-degenerate invariant bilinear form. The $J_{a}$ are called currents of this Lie algebra. When the latter is semi-simple, we shall see that the Fourier components of its currents generate the corresponding affine KacMoody algebra.

As an example, consider the theory of $N$ free fermions, with $n$-point functions given by eq. (111). Here $F(1)$ is spanned by the fields

$$
J_{i j}=N_{0}\left(\psi^{i} \psi^{j}\right),
$$

$i, j=1, \ldots, N, i<j$. One easily calculates that their OPE has the form (17) where $a, b, c$ stand for pairs $(i j)$, the invariant bilinear form is given by $d_{a b}=\delta_{a b}$, and the $f_{a b c}$ are the standard structure constants of the Lie algebra so $(N)$.

More generally, we are particularly interested in the eq. (17) when the $f_{a b c}$ are the normalised structure constants of a simple Lie algebra $X$ and

$$
d_{a b}=k \delta_{a b}
$$

for $k=1,2, \ldots$. We call the corresponding space of fields $F_{X}^{k}$. We just saw that $F_{s o(N)}^{1} \subset F^{N}$, where $F^{N}$ is the field space for $N$ free fermions. One even can show that

$$
F_{s o(N)}^{1}=F_{b}^{N} \text {. }
$$

For fixed fields $\phi^{i}, i=1, \ldots, n$ and $z_{i}$ away from the partial diagonals and 0 , the $n$-point function values 


$$
\left\langle\phi^{1}\left(z_{1}\right) \ldots \phi^{n}\left(z_{n}\right) \phi(0)\right\rangle
$$

can be interpreted as a linear form acting on $\phi \in F$. In physics it is traditional to distinguish the space $H$ spanned by the $\phi(0) 1$ from $F$ and to call the map $\phi \mapsto \phi(0) 1$ field-state identity. Indeed, in the free fermion case we first constructed $H$ and then $F$. It is easy to show that one has an isomorphism, since the $n$-point functions of $\phi$ must not vanish identically and are translationally invariant. Sometimes one wants to be $H$ a completion of $F$, but we do not need that. If you want, put $H=F$.

For holomorphic $\phi$ the maps $N_{m}(\phi,):. F \rightarrow F$ yield operators on $H$. These are the Fourier components $\phi_{m+h}$ of $\phi$. They increase the degree of a vector by $m+h$, where $h=h(\phi)$. In the literature the sign of the index often is inverted, such that this operator is denoted by $\phi_{-m-h}$, but this seems unnecessarily confusing. In any case one obtains this operator by evaluating the field $\phi$ on $z^{-m}$, with respect to the measure $d x /(2 \pi)=d z /(2 \pi i z)$. In our convention, the field-state identity is given by $\phi \mapsto \phi_{h(\phi)} 1$.

For holomorphic fields we now can move from the language of euclidean field theory to the one of operator valued distributions and back. In euclidean field theory everything is commutative, but the singularities of an $n$-point function yield non-vanishing commutators for the limiting distribution $\left\langle\phi^{1} \ldots \phi^{n}\right\rangle$ on the $n$-th Cartesian power of the unit circle. Recall that the limit to $\left|z^{k}\right|=1$ for $k=1, \ldots, n$ has to be taken from the domain with $\left|z_{1}\right|>\ldots>\left|z_{n}\right|$. Evaluating the distribution on monomials in the $z_{i}$ yields the matrix elements

$$
\left\langle\phi_{k_{1}}^{1} \ldots \phi_{k_{n}}^{n}\right\rangle=\left\langle\phi^{1} \ldots \phi^{n}\right\rangle\left(\prod_{i} z_{i}^{-k_{i}+h_{i}}\right),
$$

where $h_{i}=h\left(\phi^{i}\right)$. By Cauchy's theorem this expression can be evaluated before the limit is taken, by integrating along circles with ordered radii $\left|z_{1}\right|, \ldots,\left|z_{n}\right|$. Thus one obtains the Laurent expansion of the $n$-point function in the domain $\left|z_{1}\right|>\ldots>\left|z_{n}\right|$ by inserting

$$
\phi^{i}(z)=\sum_{m} \phi_{m}^{i} z^{m-h_{i}}
$$

keeping the order of the fields. When one changes the order of the operators $\phi_{m}^{k}$, the vacuum expectation value is given by integrating the same $n$-point function along differently ordered circles. By Cauchy's theorem, the difference of the two expressions only depends on the singularities of the function, in other words on the singular part of the OPE.

In a holomorphic tensor product theory with $F=F^{1} \otimes F^{2}$ let $\phi^{i} \in F^{i}$, $i=1,2$. The OPE for $\phi^{1}(z) \phi^{2}(w)$ has no singularities, such that $\left[\phi_{m}^{1}, \phi_{n}^{2}\right]=0$ for all $m, n$. Given an OPE closed subspace $G \subset F$ of an arbitray holomorphic theory, one can find a maximal complement $G^{\prime} \subset F$, such that one has an embedding $G \otimes G^{\prime} \subset F$. Note the $G^{\prime}$ is closed under the OPE and yields a new 
holomorphic field theory. A field $\chi$ belongs to $G^{\prime}$, iff its OPE with arbitrary fields in $F$ is non-singular, or equivalently, iff the Fourier components commute. This procedure is very important for the construction of new theories and will be used later.

For currents $J_{a}$ with an OPE (17) Cauchy's theorem yields

$$
\left[J_{a m} J_{b n}\right]=n d_{a b} \delta_{m+n, 0}+f_{a b c} J_{c, m+n} .
$$

Obviously the $J_{a 0}$ span a finite dimensional Lie algebra with structure constants $f_{a b c}$. When this Lie algebra is simple, the $J_{a n}$ and 1 span the corresponding affine Kac-Moody Lie algebra. We shall use some properties of this algebra without derivation. A standard reference is VK90.

In the case of the tensor product $F^{N}$ of $N$ free fermion theories, the so $(N)^{(1)}$ Kac-Moody algebra is represented on $H_{N S}$ and $H_{R}$. The $\mathbb{Z}_{2}$ grading of $H_{N S}$ given by $F^{N}=F_{b}^{N} \oplus F_{f}^{N}$ yields a decomposition into two subrepresentations, which turn out to be irreducible. The representation on $H_{R}$ will be considered in more detail below. The $\mathbb{Z}_{2}$ grading again yields two irreducible subrepresentations, which are isomorphic for odd $N$. In any case one obtains all irreducible level 1 representations of $s o(N)^{(1)}$.

We now had a glimpse of the interesting applications of the operator product expansion and more will come later. I think that the OPE is one of the major contributions of 20th century physics to mathematics, but still one of the major stumbling blocks when mathematicians try to learn quantum field theory. Indeed on first sight it is hard to believe that it makes sense. Take a small vector space $F$ and write any set of candidates for its $n$-point functions. Then take the OPE closure. On first sight it is hard to believe that this can yield a managable space of local fields. For holomorphic theories this is now well understood, but that it happens in many more interesting examples is one of the miracles of quantum field theory.

The particular OPE for $\phi(z) I$ yields derivative fields. For holomorphic $\phi$ one has $\partial^{m} \phi=N_{m}(\phi, I) / m !$, such that

$$
\langle(\partial \phi)(z) \chi(w)\rangle=\partial_{z}\langle\phi(z) \chi(w)\rangle .
$$

Note that $h(\partial \phi)=h(\phi)+1$. The generalization to arbitrary fields and antiholomorphic derivatives is immediate. Eq. (12) yields

$$
\partial N_{m}(\phi, \chi)=N_{m}(\partial \phi, \chi)+N_{m}(\phi, \partial \chi) .
$$

In conformally invariant theories one demands the existence of Virasoro fields. They are defined by

$$
N_{-1}(T, \phi)=\partial \phi
$$

for all $\phi \in F$. Obviously one needs $h(T)=2, \bar{h}(T)=0$. To check that the definition makes sense, let $N_{-1}\left(T, \phi_{i}\right)=\partial \phi_{i}$ for $i=1,2$, and let $\phi_{1}$ be holomorphic. By acting with $\oint T(z) d z$ on the OPE and applying Cauchy's 
theorem one finds indeed that $N_{-1}(T, \phi)=\partial \phi$ for $\phi=N_{m}\left(\phi_{1}, \phi_{2}\right)$ and any $m$. The map $\phi \mapsto N_{-2}(T, \phi)$ is grade preserving. In all examples considered here it is diagonalizable and coincides with the grading,

$$
N_{-2}(T, \phi)=h(\phi) \phi .
$$

Indeed, by acting with $\oint z T(z) d z$ on the OPE and use of Cauchy's theorem, we see that $N_{-2}\left(T, \phi_{i}\right)=h_{i} \phi_{i}$ for $i=1,2$ implies $N_{-2}(T, \phi)=\left(m+h_{1}+h_{2}\right) \phi$ for $\phi=N_{m}\left(\phi_{1}, \phi_{2}\right)$ and any $m$.

For historical reasons, the Fourier components of $T$ are called $L_{m}$. Thus the grading operator is $L_{0}$. One always assumes that the spectrum of $L_{0}$ is bounded from below. In physical terminology, one considers highest weight representation, though 'lowest weight' would make more sense. States of lowest degree are often called ground states.

In a CFT with non-holomorphic fields we also have an anti-holomorphic Virasoro field $\bar{T}$ with Fourier components $\bar{L}_{m}$, such that $\bar{L}_{0} \phi=\bar{h}(\phi) \phi$. The scaling dimension is the eigenvalue of $L_{0}+\bar{L}_{0}$. It describes the behaviour of the $n$-point functions under the transformations $z \mapsto a z$ with real positive $a$. This corresponds to time translations of the theory on $S^{1} \times \mathbb{R}$, up to an additive constant $-c / 24$, which will be explained later. The eigenvalues of $L_{0}-\bar{L}_{0}$ have to be integral and describe the effect of transformations $z \mapsto a z$ with $|a|=1$. The latter correspond to rotations of $S^{1}$, i.e. to translations of the angle $x$.

For the free fermion field $\psi$ one finds

$$
\psi(z) \psi(w)=(z-w)^{-1} I+2(z-w) T(w)+O\left((z-w)^{3}\right) .
$$

Equivalently, $T=N_{1}(\psi, \psi) / 2=N_{0}(\partial \psi, \psi) / 2$. For the OPE of $T$ one obtains

$$
T(z) T(w)=\frac{1}{4}(z-w)^{-4} I+2(z-w)^{-2} T(w)+(z-w)^{-1} \partial T(w)+O(1)
$$

or more suggestively

$$
T(z) T(w)=\frac{1}{4}(z-w)^{-4} I+(z-w)^{-2}(T(z)+T(w))+O(1) .
$$

If we write the Virasoro OPE in this symmetric way, no odd powers of $z-w$ can occur. The term proportional to $(z-w)^{-2}$ is fixed by $N_{-2}(T, T)=2 T$. The term proportional to $(z-w)^{-4}$ has conformal dimension 0 , thus must be a constant. Thus the OPE of any Virasoro field has the form

$$
T(z) T(w)=\frac{c}{2}(z-w)^{-4} I+(z-w)^{-2}(T(z)+T(w))+O(1)
$$

where $c$ is a constant, called the central charge of the theory. For the free fermion we just found $c=1 / 2$.

The tensor product of two CFTs with Virasoro fields $T^{1}, T^{2}$ is a CFT with Virasoro field $T^{1}+T^{2}$. The central charges add up. When $F$ is a holomorphic 
theory with Virasoro field $T$ and $G \subset F$ a theory with Virasoro field $T^{1}$, then the complementary subtheory $G^{\prime}$ has a Virasoro field $T-T^{1}$.

Consider now the holomorphic field theory $F_{X}^{k}$ defined by eqs. (17) and (19). Recall that $F(1)$ is spanned by the currents $J_{a}$ and that $X$ itself is given by the commutators of the $J_{a 0}$. The space $F(2)$ of fields with conformal dimension 2 is spanned by the derivatives $\partial J_{a}$ and the bilinears $N_{0}\left(J_{a}, J_{b}\right)$. Only a one-dimensional subspace of $F(2)$ is invariant under $X$, namely the multiples of $\sum_{a} N_{0}\left(J_{a}, J_{a}\right)$. A particular multiple is a Virasoro field, with central charge related to the Coxeter number $h(X)$ by

$$
c(X, k)=\frac{k \operatorname{dim}(X)}{k+h(X)} .
$$

The calculation is straightforward, but somewhat lengthy and we will no consider it here. In the case of $\mathrm{ADE}$ algebras $X$ of rank $r(X)$ we have

$$
\operatorname{dim}(X)-r(X)=r(X) h(X)
$$

such that $c(X, 1)=r(X)$. This is the only case we will consider in this article.

Let $J_{a}$ with $a=1, \ldots, r(X)$ generate a maximal abelian subalgebra of $X$. The OPE completion of their span has a Virasoro field

$$
T=\sum_{a=1}^{r(X)} \frac{1}{2} N_{0}\left(J_{a}, J_{a}\right)
$$

with central charge $r(X)$. This is the same as $c(X, 1)$, and one obtains the surprising result that the Virasoro field of this subtheory is identical to the Virasoro field of $F_{X}^{1}$, and independent of the choice of the maximal abelian subalgebra. This is an important special feature of the ADE cases. One can express it in the form that the complement of the subtheory is trivial. The calculation which yields central charge $r(X)$ is an easy and instructive exercise.

Now consider $k$ copies of $F_{X}^{1}$ and the product theory on $\left(F_{X}^{1}\right)^{\otimes k}$. Let $J_{a}^{i}$, $i=1, \ldots, k$ be the currents of the factor theories. There is a natural embedding $F_{X}^{k} \rightarrow\left(F_{X}^{1}\right)^{\otimes k}$, where the image of $F_{X}^{k}(1)$ is spanned by the currents

$$
\hat{J}_{a}=\sum_{i=1}^{k} J_{a}^{i} .
$$

Let $\tilde{F}_{X}^{k}$ be the complement of this subtheory. For its central charge one obtains immediately

$$
\tilde{c}(X, k)=\frac{k(k-1) r(X)}{k+h(X)} .
$$

Let $F_{a b}$ (with $a b$ for abelian) be the subtheory generated by the $\hat{J}_{a}$ for $a=$ $1, \ldots, r(X)$. Its central charge is $r(X)$ and the central charge of its complement 
$F_{a b}^{\prime}$ in $\left(F_{X}^{1}\right)^{\otimes k}$ is $(k-1) r(X)$. The complement $\check{F}_{X}^{k}$ of $\tilde{F}_{X}^{k}$ in $F_{a b}^{\prime}$ has central charge

$$
\check{c}(X, k)=\frac{h(X)(k-1) r(X)}{k+h(X)} .
$$

The $\check{F}_{X}^{k}$ theory also can be described as the complement of $F_{a b}$ in $F_{X}^{k}$.

We now have constructed most of the examples of CFTs which we will need to illustrate the relations to algebraic K-theory. The remaining examples are minimal models. These are rational CFTs which are the OPE closures of their respective Virasoro fields. In such a theory one has $F(1)=0$ and $F(2)=\langle T\rangle$.

Taking Fourier components and using Cauchy's theorem for the Virasoro OPE yields the Virasoro algebra

$$
\left[L_{m} L_{n}\right]=(n-m) L_{m+n}+\frac{c}{12}\left(n^{3}-n\right) \delta_{m+n, 0} .
$$

Minimal models can be described by the representation theory of this algebra, but we shall follow the euclidean formulation, which provides instructive exercises for newcomers in quantum field theory. Because of their OPE, the residues of the $n$-point function for $n$ Virasoro fields are given by the $(n-1)$ point function. Moreover, the functions vanish at infinity, so they can be calculated inductively. For the two-point function one finds

$$
\langle T(z) T(w)\rangle=\frac{c}{2}(z-w)^{-4}
$$

for the three-point function

$$
\langle T(z) T(w) T(u)\rangle=c((z-w)(w-u)(u-z))^{-2}
$$

and for the four point functions

$$
\begin{aligned}
\langle T(z) T(w) T(u) T(v)\rangle= & F(z, w, u, v)+F(z, u, w, v)+F(z, v, w, u) \\
& +G(z, w, u, v)+G(z, u, w, v)+G(z, w, v, u)
\end{aligned}
$$

where

$$
F(z, w, u, v)=\langle T(z) T(w)\rangle\langle T(u) T(v)\rangle
$$

and

$$
G(z, w, u, v)=c((z-w)(w-u)(u-v)(v-z))^{-2} .
$$

For a rapid calculation involving normal ordered products, let

$T(z) T(w)=\frac{c}{2}(z-w)^{-4} I+(z-w)^{-2}(T(z)+T(w))+\Phi(w)+O\left((z-w)^{2}\right)$.

This yields

$$
\langle T(z) \Phi(w)\rangle=-2 c(z-w)^{-6}
$$

and 
$\langle\Phi(w) T(u) T(v)\rangle=\frac{c(c+2)}{2}((w-u)(w-v))^{-4}-4 c(w-u)^{-3}(w-v)^{-3}(u-v)^{-2}$.

Note that $\Phi=N_{0}(T, T)-\partial^{2} T / 2$. With

$$
U=N_{0}(T, T)-\frac{3}{10} \partial^{2} T
$$

one finds $\langle T(z) U(w)\rangle=0$ and

$$
\langle U(w) T(u) T(v)\rangle=\frac{c}{2}\left(c+\frac{22}{5}\right)((w-u)(w-v))^{-4} .
$$

Field theories which are generated by a Virasoro field alone are called minimal models. In a minimal model the space of fields of degree at most 5 is spanned by $I, T, \partial T, \partial^{2} T, \partial^{3} T, U$ and $\partial U$. These are the fields which can occur in the singular part of the OPE for $T(w) U(z)$. For $c=-22 / 5$ the preceding formulas show, however, that this singular part cannot involve $I, T$ or its derivatives. Thus the singular terms in the Laurent expansion of $\left\langle U(z) T\left(w_{1}\right) \cdots T\left(w_{n}\right)\right\rangle$ around $z=w_{n}$ are given by $\left\langle U(z) T\left(w_{1}\right) \cdots T\left(w_{n-1}\right\rangle\right.$. Since these functions go to zero for large $z$, they vanish by induction in $n$. Thus in the minimal model with $c=-22 / 5$ any $n$-point function involving $U$ vanishes and we conclude that $U=0$. In a sense, this means that $c=-22 / 5$ yields the simplest minimal model.

The value $-22 / 5$ looks a bit strange, so let us explain the pattern of central charges in minimal models. Minimal models are classified by pairs $p, q \in \mathbb{N}$ such that $1<p<q$, and $p, q$ have no common divisor. Their central charge is $c=1-6(p-q)^{2} / p q$. The pair $(2,3)$ yields a trivial theory with $c=0$ and $H=\mathbb{C}$. The pair $(2,5)$ yields $c=-22 / 5$, whereas the pair $(3,4)$ yields the bosonic part of the free fermion theory and $c=1 / 2$.

For perturbation theory we will need more complicated non-holomorphic fields than those considered so far, but for the purpose of this article we need little more to know about them than their conformal dimensions. These are constrained by the algebra of the holomorphic fields in a way which is best discussed in terms of representation theory. According to the field-state correspondence, all fields should correspond to vectors $v \in H$. For arbitrary homogeneous vectors $v_{1} \in H^{*}, v_{2} \in H$ and holomorphic fields $\phi^{k}$ we can study the generalized $n$-point functions $\left\langle v_{1}\left|\phi^{1}\left(z_{1}\right) \ldots \phi^{n}\left(z_{n}\right)\right| v_{n}\right\rangle$. The Laurent expansions around the partial diagonals are given by the OPE of the holomorphic fields, which can be read off from the ordinary $n$-point functions, as discussed above. When all fields $\phi^{k}$ are bosonic or when $v_{1}, v_{2}$ belong to the NS sector, the generalized $n$-point functions have the same analytic properties as the ordinary ones, except for the fact that poles also occur at $z_{i}=0$ and $z_{i}=\infty$. The order of the pole at $z_{i}=0$ is bounded by $h\left(\phi^{i}\right)+c_{2}$, where $c_{2}$ is a constant given by the holomorphic degree of $v_{2}$ and independent of $n$ and of the choice of fields. Similarly, the pole order at $\infty$ is bounded by $h\left(\phi^{i}\right)+c_{1}$, with an analogous constant $c_{1}$. 
When $v_{1}, v_{2}$ belong to the $\mathrm{R}$ sector and $\psi \in F_{f}$, then $\left\langle v_{1} \mid \psi(z) \ldots\right\rangle$ changes sign when $z$ moves around the origin. To recover the standard analytic behaviour of the generalized $n$-point functions we have to multiply $\left\langle v_{1}\left|\phi^{1}\left(z_{1}\right) \ldots \phi^{n}\left(z_{n}\right)\right| v_{n}\right\rangle$ by the product of all $\sqrt{z_{k}}$ for which $\psi_{k} \in F_{f}$. When $v_{1}$ belongs to the NS sector and $v_{2}$ to the $\mathrm{R}$ sector, the functions have to vanish.

Given an OPE of holomorphic fields $F$, we can constrain the vectors $v \in H$, or rather the vectors $v_{1} \otimes v_{2} \in H^{*} \otimes H$ by abstracting from these properties. Let $\mathcal{F}(n)$ be the space of meromorphic functions on $\mathbb{C}^{n}$ with poles only on the partial diagonals, 0 and infinity. A NS state on the OPE is a map from the tensor algebra $T(F)$ to $\oplus_{n} \mathcal{F}(n)$ such that the Laurent expansions around the partial diagonals are given by the OPE and such that the order of the poles at 0 and $\infty$ obeys the restrictions mentioned above. Such a map will be interpreted as a system of generalized $n$-point functions $\left\langle v_{1}\left|\phi^{1}\left(z_{1}\right) \ldots \phi^{n}\left(z_{n}\right)\right| v_{2}\right\rangle$. The $\mathrm{R}$ states on the OPE are defined analogously. Of course the distinction between NS and R representations only makes sense for $F_{f} \neq 0$. In this case, the basic representation on $F$ itself is a NS representation.

The Laurent expansions around $z=0$ and $z=\infty$ of the $(n+1)$-point functions $\left\langle v_{1}\left|\phi(z) \phi^{1}\left(z_{1}\right) \ldots \phi^{n}\left(z_{n}\right)\right| v_{2}\right\rangle$ yield states $v_{1} \otimes\left(\phi_{m} v_{2}\right)$ and $\left(v_{1} \phi_{m}\right) \otimes v_{2}$ on the OPE. Thus every state generates a vector space of states with right and left actions of the Fourier components of the holomorphic fields. This vector space of states will be called a representation of the OPE. The space can be graded by the constants $c_{1}, c_{2}$ introduced above. For rational theories, its homogeneous subspaces have finite dimensions. The definition of direct sums and of irreducible representations is the standard one. For irreducible representations, the vector space of states can be written as $H_{i}^{*} \otimes H_{i}$, and as usual we identify $H_{i}$ with the representation space. When one works harder, one can define tensor products of representations, but we will not need them.

In general a holomorphic OPE has various irreducible representations. A simple example is given by the basic representation of any theory with $F_{f} \neq 0$. By the field-state identity $H \simeq F_{b} \oplus F_{f}$. When restrict $F$ to $F_{b}$, the representation on $F_{b} \oplus F_{f}$ decomposes into irreducible representations on $F_{b}$ and on $F_{f}$. The first one is the new basic representation, since $I \in F_{b}$. The representations cannot be isomorphic, since $F_{b}$ is graded by the integers, whereas the eigenvalues of $L_{0}$ in $F_{f}$ are half-integral.

For a vector $v$ of lowest degree $h$ in any representation one has $L_{m} v=0$ for $m<0$, since there are no vectors of degree $m+h$. With $L_{0} v=h v$ one finds for normalised $v$

$$
\langle v|T(z)| v\rangle=h z^{-2}
$$

and

$$
\langle v|T(z) T(w)| v\rangle=\frac{c}{2}(z-w)^{-4}+2 h(z w)^{-1}(z-w)^{-2}+h^{2}(z w)^{-2} .
$$

The calculation of $\langle v|U(z)| v\rangle$ from this formula and eq. (22) is easy and left to the reader. Using $U=0$ in the $(2,5)$ minimal model yields $h(h+1 / 5)=0$. 
Indeed one can construct two representations of the OPE of this model, the basic representation with $h=0$ and another one with $h=-1 / 5$. Irreducible representations of minimal models are determined up to isomorphism by the lowest eigenvalue of $L_{0}$, but this will not be shown here.

The free fermion theory has a unique irreducible Ramond representation. Recall that $\sqrt{z w}\left\langle v_{1}|\psi(z) \psi(w)| v_{2}\right\rangle$ is meromorphic, with a single pole at $z=w$ of residue $\left\langle v_{1} \mid v_{2}\right\rangle$. Taking into account the antisymmetry under exchange of $z, w$ this yields for a normalised Ramond groundstates $v=v_{1}, v_{2}$

$$
\sqrt{z w}\langle v|\psi(z) \psi(w)| v\rangle=\frac{z+w}{2(z-w)} .
$$

For the Virasoro field $T$ one obtains

$$
\langle v|T(z)| v\rangle=(4 z)^{-2}
$$

thus

$$
L_{0} v=\frac{1}{16} v
$$

The bosonic part $F_{b}$ of the free fermion theory yields the $(3,4)$ minimal model with $c=1 / 2$. In the NS case, $H$ decomposes into a direct sum of the basic representation $F_{b}$ with $h=0$ and $F_{f}$ with $h=1 / 2$. We just have seen that the $\mathrm{R}$ case yields a representation with $h=1 / 16$. Using similar arguments as for $c=-22 / 5$ one can show that these are the only irreducible representations.

When one has $N$ free fermions, the $R$ ground states have $h=N / 16$ and form the spin representation of the $s o(N)$ Lie algebra obtained above. For even $N$ the dimension of this space is $2^{N}$ and the $\mathbb{Z}_{2}$-decomposition $H_{R}=H_{R}^{0} \oplus H_{R}^{1}$ yields half-spinor representations on the ground states of $H_{R}^{0}$ and $H_{R}^{1}$. Similaly, one has the $\mathbb{Z}_{2}$-decomposition $H_{N S}=H_{N S}^{0} \oplus H_{N S}^{1}$ given by $F^{N}=F_{b}^{N} \oplus F_{f}^{N}$. For $N=16$, the fields corresponding to the $R$ groundstates have $h=1$, and one can find a new holomorphic theory with $F \simeq H_{N S}^{0} \oplus H_{R}^{0}$. One has

$$
\operatorname{dim} F(1)=120+2^{7},
$$

so it is not hard to guess that this is the theory of $E_{8}$ currents with OPE (17) and $d_{a b}=\delta_{a b}$. The Virasoro field of this theory lies in $H_{N S}^{0}$ and restricts to the Virasoro field of the $s o(16)$ theory, with $c=8$.

When one takes two copies of this theory, the sums of the currents generate a subtheory, and by eq. (201) the complementary theory has $\tilde{c}\left(E_{8}, 2\right)=1 / 2$. Indeed it is isomorphic to the $(3,4)$ minimal model. Thus there is a close link between this minimal model and $E_{8}$. This should make eq. (5) somewhat less miraculous.

An important property of conformally invariant quantum field theories is the possibility to transfer them to arbitrary compact Riemann surfaces. This is possible since operator product expansions are local and can be rewritten in terms of conformally equivalent local coordinates. In particulars one can 
find a new Virasoro field $\tilde{T}$ adapted to the changed coordinates. Let us put $z=f(x), w=f(y)$ and evaluate $\tilde{T}$ with respect to $x$. One has

$$
\begin{aligned}
(z-w)^{2}= & f^{\prime}(x) f^{\prime}(y)(x-y)^{2} \\
& -\frac{1}{24}\left(2 f^{\prime}(x) f^{\prime \prime \prime}(x)-3 f^{\prime \prime}(x)^{2}+2 f^{\prime}(y) f^{\prime \prime \prime}(y)-3 f^{\prime \prime}(y)^{2}\right)(x-y)^{4} \\
& +O\left((x-y)^{6}\right) .
\end{aligned}
$$

By insertion in the OPE of $T$, one easily confirms that

$$
\tilde{T}(x)=T(z)(d z / d x)^{2}+\frac{c}{12} S(f)
$$

where $S(f)=\left(f^{\prime} f^{\prime \prime \prime}-3\left(f^{\prime \prime}\right)^{2} / 2\right)\left(f^{\prime}\right)^{-2}$ is the Schwarzian derivative. For $z=$ $\exp (i x)$ we have $S(f)=1 / 2$, which shows that the holomorphic part of the generator of time translations on $S^{1} \times \mathbb{R}$ is given by

$$
-\int \tilde{T}(x) \frac{d x}{2 \pi}=L_{0}-c / 24 .
$$

By the OPE, $n$-point functions on arbitrary compact Riemann surfaces can be calculated in terms of the 1-point functions. The one point functions on a torus with periods $(2 \pi, 2 \pi \tau)$ and $q=\exp (2 \pi i \tau)$ have the form

$$
\langle\phi\rangle_{\tau}=\operatorname{Tr}\left(q^{L_{0}-c / 24} \bar{q}^{\bar{L}_{0}-\bar{c} / 24} \phi(x)\right),
$$

and are independent of $x$.

For $\phi=I$ one obtains the partition function $Z(\tau)=\langle I\rangle_{\tau}$. By conformal invariance, $Z$ only depends on the ratio $\tau$ of the torus periods. By choosing a different set of generators of the torus periods, this ratio can be changed to $(A \tau+B) /(C \tau+D)$, where $\left(\begin{array}{l}A B \\ C D\end{array}\right) \in S L(2, \mathbb{Z})$. This explains the modular invariance of the partition functions of bosonic CFTs. In the fermionic case the spin structures of the torus have to be taken into account. We do not need to discuss this case, since one always can restrict a fermionic CFT to its bosonic part.

For rational CFTs there are finite sets of irreducible representations for the OPEs of the holomorphic and the anti-holomorphic fields. Let the corresponding representation spaces be labelled by $V_{i}, \bar{V}_{j}$, where $V_{0}, \bar{V}_{0}$ are the basic representations to which the vacuum belongs. Let $V_{i} \otimes \bar{V}_{j}$ occur with multiplicity $n_{i j}$ in $H$. Then

$$
Z=\sum_{i, j} n_{i j} \chi_{i} \bar{\chi}_{j}
$$

where

$$
\chi_{i}(\tau)=\operatorname{Tr}_{V_{i}} q^{L_{0}-c / 24}
$$


and analogously for $\bar{\chi}_{j}$. This explains eqs. (1), (2).

For the free fermion OPE, we had found a NS representation on a space $V_{N S}$ and a $\mathrm{R}$ representation on a space $H_{0} \otimes V_{R}$ where $V_{N S}, V_{R}$ are spanned by vectors of the form $e_{k_{1}} \wedge e_{k_{2}} \wedge \cdots e_{k_{n}}$ with $k_{1}>k_{2}>\cdots k_{n}>0$ and $e_{k}=\exp (-i k x)$, with half-integral and integral $k$, resp. The $\mathbb{Z}_{2}$ degree of such a vector is 0 for even $n$ and 1 for odd $n$. The two-dimensional space $H_{0}$ has degree 0 and one-dimensional even and odd subspaces. This yields characters

$$
\begin{aligned}
& \chi_{N S}^{(3,4)}=q^{-1 / 48} \prod_{r=1 / 2,3 / 2, \ldots}\left(1+q^{r}\right) \\
& \chi_{R}^{(3,4)}=2 q^{1 / 24} \prod_{n=1}^{\infty}\left(1+q^{n}\right),
\end{aligned}
$$

where we used $c=1 / 2$ and in the Ramond case the fact that $L_{0}$ has eigenvalue $1 / 16$ on the ground state. The factor of 2 in $\chi_{R}^{(3,4)}$ comes from the $\mathbb{Z}_{2}$ grading.

When we restrict the OPE algebra of the holomorphic free fermion theory to its bosonic part, one obtains the OPE of the $(3,4)$ minimal model. The decomposition of the free fermion characters into irreducible $(3,4)$ characters is given by

$$
\begin{aligned}
& \chi_{N S}^{(3,4)}=\chi_{0}^{(3,4)}+\chi_{2}^{(3,4)} \\
& \chi_{R}^{(3,4)}=2 \chi_{1}^{(3,4)}
\end{aligned}
$$

where

$$
\chi_{0}^{(3,4)}-\chi_{2}^{(3,4)}=q^{-1 / 48} \prod_{r=1 / 2,3 / 2, \ldots}\left(1-q^{r}\right) .
$$

Up to phases, the $\chi_{i}^{(3,4)}, i=0,1,2$, are invariant under the subgroup $\Gamma_{0}(2)$ of the modular group which is given by matrices $\left(\begin{array}{l}A B \\ C D\end{array}\right) \in S L(2, \mathbb{Z})$ with even $C$. When one combines holomorphic and anti-holomorphic parts of the full minimal $(3,4)$ CFT one obtains a torus partition function

$$
Z^{3,4}=\left|\chi_{0}^{(3,4)}\right|^{2}+\left|\chi_{1}^{(3,4)}\right|^{2}+\left|\chi_{2}^{(3,4)}\right|^{2}
$$

which is invariant under the full modular group.

Apart from their product representations, $\chi_{N S}^{(3,4)}$ and $\chi_{R}^{(3,4)}$ also have sum representations, which are more important for our present purpose. The spaces $V_{N S}$ and $V_{R}$ are direct sums of subspaces $V_{N S}(n)$ and $V_{R}(n)$ spanned by vectors of the form $e_{k_{1}} \wedge e_{k_{2}} \wedge \cdots e_{k_{n}}$ with fixed $n$. We can put $k_{m}=k_{m+1}+$ $1+s_{m}$, where $k_{n+1}=b-1 / 2$ and $b=0$ in the NS and $b=1 / 2$ in the R case. Then

$$
\sum_{m=1}^{n} k_{m}=n^{2} / 2+b n+\sum_{m=1}^{n} m s_{m} .
$$


The $s_{m}$ are independent and take values from 0 to $\infty$. Thus

$$
\operatorname{Tr}_{V_{R}(n)} q^{L_{0}-1 / 16}=\frac{q^{n(n+1) / 2}}{(q)_{n}}
$$

and

$$
\operatorname{Tr}_{V_{N S}(n)} q^{L_{0}}=\frac{q^{n^{2} / 2}}{(q)_{n}} .
$$

Here $(q)_{n}=(1-q)\left(1-q^{2}\right) \cdots\left(1-q^{n}\right)$ is the so-called $q$-deformed factorial. For small $\tau$ it behaves like $(-2 \pi i \tau)^{n} n$ !, which explains the name.

For the $(2,5)$ minimal model, one also finds characters with nice representations as sums and products. The space of all holomorphic fields is spanned by expressions of the form

$$
\partial^{m_{n}} T \ldots \partial^{m_{2}} T \partial^{m_{1}} T
$$

where $m_{n} \geq \ldots \geq m_{1}$ and the normal ordering is suppressed. By eq. (22) and $U=0$ we have

$$
T(z) T(w)=-\frac{3}{10} \partial^{2} T(w)+O(z-w) .
$$

Taking derivatives $\left(\partial_{z}+\partial_{w}\right)^{2 n}$ and $\left(\partial_{z}+\partial_{w}\right)^{2 n-1}$ of this equation, one sees that the normal ordered products $N_{0}\left(\partial^{n} T, \partial^{n} T\right)$ and $N_{0}\left(\partial^{n} T, \partial^{n-1} T\right)$ are linear combinations of terms which are simpler in a suitable lexikographical order. One can show that no further linear dependencies exist. Thus the character in the vacuum sector is given by the sum over all expressions of the form

$$
q^{2+m_{n}} \ldots q^{2+m_{2}} q^{2+m_{1}}
$$

where $m_{k} \geq m_{k-1}+1$ and $m_{1} \geq 0$. Together with the factor $q^{-c / 24}$ this yields

$$
\chi_{0}^{(2,5)}=q^{11 / 60} \sum_{n \in \mathbb{N}} \frac{q^{n(n+1)}}{(q)_{n}} .
$$

In the sector with ground state $v$ and $L_{0} v=-v / 5$ one finds an anlogous formula. Here $L_{1} v$ does not vanish, but again terms with $L_{n} L_{n}$ and $L_{n} L_{n-1}$ are linear combinations of simpler terms. Thus the character in this sector is given by the sum over all expressions of the form

$$
q^{1+m_{n}} \ldots q^{1+m_{1}} q^{1+m_{1}},
$$

where $m_{k} \geq m_{k-1}+1$ and $m_{1} \geq 0$. Together with the factor $q^{-c / 24-1 / 5}$ this yields

$$
\chi_{1}^{(2,5)}=q^{-1 / 60} \sum_{n \in \mathbb{N}} \frac{q^{n^{2}}}{(q)_{n}} .
$$


Since the work of Rogers and Ramanujan it is well known that $\chi_{0}^{(2,5)}$ and $\chi_{1}^{(2,5)}$ are modular and have the product expansions (44). The partition function

$$
Z^{2,5}=\left|\chi_{0}^{(2,5)}\right|^{2}+\left|\chi_{1}^{(2,5)}\right|^{2}
$$

is invariant under all modular transformations.

The sum forms of the $(2,5)$ characters look very similar to the the free fermion ones. Can they be generalized? In both cases one has expressions

$$
\sum_{n \in \mathbb{N}} \frac{q^{a n^{2} / 2+b n+h}}{(q)_{n}},
$$

where $a$ characterizes the CFT, and $b, h$ change according to the sector of the theory. When the tensor product of $r$ models is formed, one obtains the product of the corresponding characters, thus

$$
\chi=\sum_{n \in \mathbb{N}^{r}} q^{n A n / 2+b n+h} /(q)_{n},
$$

where now $A$ is a diagonal $r \times r$ matrix, $b \in \mathbb{Q}^{r}$ and $b n$ is to be understood as scalar product in $\mathbb{Q}^{r}$. Finally

$$
(q)_{n_{1}, \ldots, n_{r}}=\prod_{i=1}^{r}(q)_{n_{i}} .
$$

As has been mentioned in the introduction, one can find other characters of conformally invariant quantum field of the form (6), but with a non-diagonal matrix $A$. For the $(2, q)$ minimal models one obtains the Andrews-Gordon generalization of the Rogers-Ramanujan identities, and for $A=2 \mathcal{C}\left(E_{8}\right)^{-1}$ and $\mathcal{C}\left(E_{8}\right)$ the Cartan matrix of $E_{8}$ one obtains (5) as an equation for $\chi_{0}^{(3,4)}$.

To see how the matrices $A$ arise from a given conformal field theory, we have to look at its massive perturbations. This is done in the next section. It needs more physics and leads somewhat beyond the domain of present-day rigorous mathematics. A reader who prefers to accept equation (6) without physical motivation can skip the next section.

\section{Integrable Perturbations}

To explain the form (6) of characters, the corresponding CFTs have to be understood as limits of more general integrable quantum field theories. The latter are still local, but no longer conformally invariant. They are not under complete mathematical control, though integrability helps a lot. Their partition functions and some properties of their $n$-point functions are calculable, but the calculations depend on assumptions which are plausible but not proven. 
We shall proceed as follows. First we shall discuss the theory of free massive fermions as a perturbation of a conformally invariant theory of massless fermions. Since the theory is free, everything can be calculated exactly with little effort. We shall see that the $(3,4)$ minimal models has perturbations with two parameters, one given by the fermion mass just mentioned, the other one related to $E_{8}$. If only the latter one is used, the theory remains integrable, due to the existence of suitable higher conservation laws. Then we sketch the description of the state space of massive theories, the scattering matrices of integrable theories and the Bethe ansatz. The form of the characters (6) will follow and we shall see how the integrable perturbation determines the matrix $A$, at least in principle. Much more could be done, since the mathematical structure of the integrable theories is very beautiful. Its relations to algebraic K-theory have not been explored yet, however.

Nevertheless, the physical arguments explained in this section can be used as a guide for future mathematical research. Mathematicians who want to follow this lead will have to learn to read some physics textbooks. Thus this section is less self-contained than the others and uses some elementary physics background and terminology, for example from relativistic mechanics.

For free massive fermions the quantization procedure discussed at the beginning of section 2 can be used. The Dirac equation (10) couples $\psi_{L}$ and $\psi_{R}$, so both have to be considered together. Let us first do that in the massless case. We identify the holomorphic field $\psi$ used above with $\psi_{L}$. Recall that we use a splitting $V=V_{+} \oplus V_{-}$of the vector space to be quantized. The space $V_{-}$corresponds to the negative Fourier components with respect to time translation, and a correct description of time demands that this is done consistently for all fields. Now for right- and left-movers a given time translation corresponds to opposite space translations. We described the distribution $\left\langle\psi_{L}(x) \psi_{L}(y)\right\rangle$ as the $\epsilon \rightarrow+0$ limit of a function $\left\langle\psi_{L}(x) \psi_{L}(y+i \epsilon)\right\rangle$, with holomorphic dependence on $y+i \epsilon$. Thus $\left\langle\psi_{R}(x) \psi_{R}(y)\right\rangle$ is the limit of a function $\left\langle\psi_{R}(x) \psi_{R}(y+i \epsilon)\right\rangle$ which depends holomorphically on $(-y+i \epsilon)$ or anti-holomorphically on $y+i \epsilon$. The latter description allows us to obtain all distributions $\left\langle\phi^{1}\left(x_{1}, t_{1}\right) \ldots \phi^{n}\left(x_{n}, t_{n}\right)\right\rangle$ by analytic continuation of a euclidean $n$-point function with purely imaginary time components $t_{k}$. The limit is taken such that $\Im t_{1}<\ldots<\varsigma t_{n}$.

For the $n$-point functions we still can use Wick's theorem (8), with $\left\langle\psi_{L} \psi_{R}\right\rangle=0$ and

$$
\left\langle\psi_{R}(z) \psi_{R}(w)\right\rangle=(\bar{z}-\bar{w})^{-1} .
$$

Since $\psi_{R}$ is anti-holomorphic in the euclidean description, one usually uses the notation $\psi_{R}=\bar{\psi}$. We will follow this notation, though it is somewhat misleading, since $\psi_{L}$ and $\psi_{R}$ are independent fields which are both real with respect to the standard anti-involution of $F$.

Now we consider deformations of a given quantum field theory. We assume that the deformed theories are translationally invariant and have a unique vacuum vector $1 \in H$. We assume that we have euclidean $n$-point functions 
which are real analytic, apart from singularities along the partial diagonals. From the latter we read off the operator product expansion. It has the form

$$
\phi_{i}(z) \phi_{j}(w)=\sum_{k} f_{i j k}(z-w) \phi_{k}(w),
$$

with real analytic functions $f_{i j k}(z-w)$. We assume rotational invariance, such that every field $\phi_{i}$ has a conformal spin $s_{i}$ analogous to $h_{i}-\bar{h}_{i}$. This means that

$$
(z-w)^{s_{i}+s_{j}-s_{k}} f_{i j k}(z-w)
$$

only depends on the radial distance $|z-w|$. The dependence on this distance is far more complicated than in the conformally invariant case, however. In particular, the space $F$ of local fields is no longer graded by a conformal dimension $d=h+\bar{h}$. Nevertheless we assume that at short distance the breaking of conformal invariance is weak. This means that $F$ is filtered by a scaling dimension $d$, such that

$$
\left|f_{i j k}(z-w)\right|=o\left(|z-w|^{d_{k}-d_{i}-d_{j}+\epsilon}\right)
$$

for all $\epsilon>0$.

In this way one gets close to an axiomatic definition of general quantum field theories, but one is very far from calculability. Efficient calculations are possible, when a quantum field theory depends differentiably on some parameter $\lambda$, such that for $\lambda=0$ all $n$-point functions are calculable. The deformation away from $\lambda=0$ is performed by perturbation theory. In our case, the unperturbed theory will be conformally invariant.

In general, the structure of quantum field theories is so restricted that the perturbations of a given theory can be described by points in a low dimensional moduli space. We consider a single parameter $\lambda$ for ease of notation. The space of fields $F$ should be locally trivializable over the parameter space such that the $n$-point functions of the deformed theory depend real analytically on $\lambda$. The $n$-point functions are constrained by the requirement that expansions around the diagonals of $\mathbb{C}^{n}$ does not lead to additional fields.

We have an OPE

$$
\phi_{i}(z) \phi_{j}(w)=\sum_{k} f_{i j k}(z-w, \lambda) \phi_{k}(w)
$$

At the conformally invariant point $\lambda=0$, eq. (15) yields

$$
f_{i j k}(z-w, 0)=C_{i j k}(z-w)^{h_{k}-h_{i}-h_{j}}(\bar{z}-\bar{w})^{\bar{h}_{k}-\bar{h}_{i}-\bar{h}_{j}},
$$

with constant coefficients $C_{i j k}$. In a first order expansion around $\lambda=0$ this yields equations 


$$
\begin{aligned}
\partial_{\lambda}\left\langle\phi_{i}(z) \phi_{j}(w) \ldots\right\rangle= & \sum_{k} C_{i j k}(z-w)^{h_{k}-h_{i}-h_{j}}(\bar{z}-\bar{w})^{\bar{h}_{k}-\bar{h}_{i}-\bar{h}_{j}} \partial_{\lambda}\left\langle\phi_{k}(w) \ldots\right\rangle \\
& +\sum_{k}\left\langle\phi_{k}(w) \ldots\right\rangle \partial_{\lambda} f_{i j k}(z-w, 0) .
\end{aligned}
$$

These are consistency equations for the $\lambda$-derivatives of the $n$-point functions and of the OPE functions $f_{i j k}$ at $\lambda=0$. By eq. (15), the coefficients of these $\lambda$-derivatives are homogeneous under scaling, thus one also has a basis of solutions of the consistency equations which is homogeneous under scaling. In other words, we can assume that $\lambda$ itself has well defined scaling dimension $\delta, \bar{\delta}$. Since the conformal spins $h(\phi)-\bar{h}(\phi)$ are integral and cannot change, one needs $\delta=\bar{\delta}$. We assume $\delta \geq 0$, for a reason which will be explained below.

Now we consider the free massless fermion with components $\psi, \bar{\psi}$ and show that the massive Dirac equation (10) is the only possible deformation. Note that we demand that the deformed theory still has fields $\psi, \bar{\psi}$ which generate all of $F$ by normal ordered products. For non-zero $\lambda$ the local field $\bar{\partial} \psi$ no longer will vanish, but a priori one will have

$$
\bar{\partial} \psi=\lambda \chi^{1}+\lambda^{2} \chi^{2}+\ldots
$$

Comparing scaling dimensions $h$ one finds $1 / 2=k \delta+h\left(\chi^{k}\right), 1=k \delta+\bar{h}\left(\chi^{k}\right)$. The only possible solution is $\delta=1 / 2$,

$$
\bar{\partial} \psi=\lambda \bar{\psi},
$$

and analogously for $\partial \bar{\psi}$, up to a constant. Note that higher powers of $\lambda$ cannot occur, since there are no fields $\chi^{k}$ with appropriate dimensions. With a suitable rescaling of the fields the constant can be chosen to be 1 or -1 . Since euclidean $n$-point functions have to go to zero at infinity only the plus sign in $\partial \bar{\partial} \psi= \pm \lambda^{2} \psi$ is allowed, such that

$$
\bar{\partial} \psi=\lambda \bar{\psi}
$$

In this way we recover the theory of a free fermion on $S^{1} \times \mathbb{R}$ which satisfes the Dirac equation (10) with non-vanishing $\mu=\lambda$. It can be quantized by the same method as the massless case. The theory is manifestly invariant under space and time translations, thus the 2-point function of the fermion field depends on two variables $x, y$. It is easily obtainable from the Green's function of $\partial_{x}^{2}+\partial_{y}^{2}-\mu^{2}$. On $\mathbb{R}^{2}$ this is a Bessel function depending on $\mu|z-w|$. More details can be found in any textbook of quantum field theory and will not be given here. Note, however, that the short distance behaviour $z-w \rightarrow 0$ is equivalent to the massless limit $\mu \rightarrow 0$. If one considers the theory on $S^{1} \times \mathbb{R}$ instead of $\mathbb{R}^{2}$, the leading short distance singularities do not changer. This can be understood as a consequence of the locality of the theory.

For general deformations with $\delta \neq 0$, the two-point function will depend on $\lambda|z-w|^{2 \delta}$. For negative $\delta$, the short distance behaviour would be more 
singular than for the unperturbed theory, and an infinite Taylor expansion in $\delta$ would introduce short distance singularities which are stronger than any negative power of $|z-w|$. Presumably this is inconsistent, and in any case it could not be handled by available methods, so we exclude $\delta<0$. The case $\delta=0$ includes the deformations within the moduli spaces of the conformally invariant theories themselves. It is particularly important, but not in our context and will not be considered here.

We now need to answer two important questions: How does one classify perturbations with $\delta>0$ and which of them are integrable? Both questions turn out to be related to the study of derivatives. When a derivative $\partial \phi$ is nonzero, there is nothing much to discuss, one just can choose the trivialization of $F$ over the moduli space of the perturbed theory such that relations like $\partial \phi=\chi$ between $\phi, \chi \in F$ remain true when $\lambda$ is varied.

Holomorphic fields $\phi$ need not remain holomorphic, however, as we have seen. Since dimensions are bounded from below, $\bar{\partial} \phi$ is a polynomial in $\lambda$. Let us assume that this polynomial is linear, as it was the case for the free fermion fields. In particular this is enforced if the theory is unitary and $\delta>1 / 2$. Let $F(h, \bar{h})$ be the space of fields with conformal dimensions $h, \bar{h}$. We define a map

$$
\vartheta: F(h, 0) \rightarrow F(h-\delta, 1-\delta)
$$

by

$$
\bar{\partial} \phi=\lambda \vartheta \phi .
$$

A particularly important case concerns the energy momentum tensor $T, \bar{T}$. Energy and momentum must be conserved to insure translation invariance in time and space. In the coordinates $x, t$ conservation laws have the form $\partial_{t} \phi=\partial_{x} \chi$, since this implies that $\int \phi d x$ is independent of $t$. Thus one expects

$$
\begin{aligned}
\bar{\partial} T & =\lambda \partial \Phi \\
\partial \bar{T} & =\lambda \bar{\partial} \bar{\Phi} .
\end{aligned}
$$

Scaling yields $h(\Phi)=\bar{h}(\Phi)=1-\delta$ and $h(\Phi)=h(\bar{\Phi})$. The change of momentum is given by $\int(\Phi-\bar{\Phi}) d x$. Since the momentum on $S^{1}$ is quantized, this has to vanish, so $\Phi-\bar{\Phi}$ can be written as a derivative with respect to $x$. For dimensional reasons, this cannot be realized in a non-trivial way, so we need $\Phi=\bar{\Phi}$. The field $\Phi$ uniquely characterizes the deformation and each real field $\Phi$ with $h(\Phi)=\bar{h}(\Phi)<1$ generates a possible perturbation with one parameter $\lambda$. Thus we have classified the massive deformations of a CFT. For the perturbation of the free fermion theory considered above one finds $\Phi=N_{0}(\bar{\psi} \psi) / 2$, as we shall see.

Integrable theories are characterized by more conserved quantities, so we are interested in more holomophic fields which behave like $T$. In other words, we want to find fields for which the image of the map $\vartheta$ lies in $\partial F(h-1-\delta, 1-$ $\delta)$. In particular, this is true for the fields of the form $\partial \phi$ with $\phi \in F(h-1,0)$, 
but these fields yield no conserved quantities, since $\int \partial_{x} \phi d x=0$. Conversely, every field with vanishing integral over $S^{1}$ is of this form.

Put $D(h, \bar{h})=\operatorname{dim} F(h, \bar{h})$ and

$$
\Delta(h)=(D(h, 0)-D(h-1,0))-(D(h-\delta, 1-\delta)-D(h-1-\delta, 1-\delta)) .
$$

When $\Delta(h)>0$, the subspace of $F(h, 0) / \partial F(h-1,0)$ for which the image of $\vartheta$ lies in $\partial F(h-1-\delta, 1-\delta) / \partial \vartheta F(h-1,0)$ has at least dimension $\Delta(h)$. We have seen that this subspace yields conserved quantities in the perturbed theory. This is Zamolodchikov's counting argument.

In the $(3,4)$ minimal model with partition function (24) there are two fields with $h=\bar{h}<1$, namely $\psi \bar{\psi}$ and a field with conformal dimensions $(1 / 16,1 / 16)$. The latter can be used for a perturbation with $\delta=15 / 16$. The $D(h, \bar{h})$ are known from eq. (24) and one finds conserved quantities corresponding to holomorphic fields of dimensions $h_{i}=2,8,12,14,18,20$. For $h_{i}=2$ this is just the energy-momentum, but the higher conserved quantities yield an integrable theory. The relation to the Coxeter exponents $1,7,11,13,17,19,23,29$ for $E_{8}$ is obvious, and there are strong arguments for the existence of conservation laws for fields of conformal spin $m_{i}+30 n+1$, where $m_{i}$ is an $E_{8}$ Coxeter exponent and $n \in \mathbb{N}$ Z89 Z91. Note that 30 is the Coxeter number of $E_{8}$.

The conservation laws of the theory of free massive fermions follow the same pattern. In this case Zamolodchikov's counting argument does not apply. Since $\mu$ has dimensions $(1 / 2,1 / 2)$ and the perturbation of a conservation law $\bar{\partial} \phi=0$ yields

$$
\bar{\partial} \phi=\mu \chi^{1}+\mu^{2} \chi^{2}
$$

with $h\left(\chi^{2}\right)=h(\phi)-1$ and $\bar{h}\left(\chi^{2}\right)=0$. Thus it is not sufficient that $\chi^{1}$ is a derivative field. Instead, one can use the fact that the theory is free to find explicit conservation laws. One writes the relevant cases of the OPE in the form

$$
\partial^{m} \psi(z) \partial^{n} \psi(w)=\left\langle\partial^{m} \psi(z) \partial^{n} \psi(w)\right\rangle I+: \partial^{m} \psi \partial^{n} \psi:(w)+o\left(|z-w|^{0}\right),
$$

and analgously for $\bar{\psi}$. In the limit $\mu=0$, the normal ordering by :: coincides with $N_{0}$. Since $\partial^{n} \psi \in F_{f}$ one has : $\partial^{n} \psi \partial^{n} \psi:=0$ and consequently for $n \geq 1$

$$
\bar{\partial}: \partial^{n} \psi \partial^{n+1} \psi:=\mu^{2}: \partial^{n-1} \psi \partial^{n+1} \psi:=\mu^{2} \partial: \partial^{n-1} \psi \partial^{n} \psi: \text {. }
$$

For $n=0$ one finds

$$
\bar{\partial}: \psi \partial \psi:=\mu \partial: \bar{\psi} \psi:
$$

that is $\bar{\partial} T=\mu \Phi$, where

$$
\Phi=\frac{1}{2}: \bar{\psi} \psi: .
$$

Thus we have conserved quantities for fields of conformal spin $m+2 n+1$, where $n \in \mathbb{N}, m=1$ is the unique Coxeter exponent of $A_{1}$ and 2 is the Coxeter 
number of $A_{1}$. Thus the pattern of conserved quantities is analogous to the $E_{8}$ perturbation. For arbitray ADE Lie algebras $X$ one expects such a pattern for a perturbation of the theory with field space $\tilde{F}_{X}^{2}$ discussed in the previous section.

We now could go on to the description of the Bethe ansatz for calculations in integrable massive theories, but let us digress briefly to see how perturbed $n$-point functions are calculated. The main purpose of the digression is to convince mathematicians that quantum field theory may be difficult, but is certainly no black art. The eqs. (26) form a huge system, but conjecturally all solutions are known. There is a big space of trivial solutions, which just comes from the possibility to relabel the fields by acting with some $\lambda$-dependent elements of $G L(F)$. Surprisingly, this will turn out to be important, but of course we are only interested in solutions modulo the trivial ones. The interesting solutions are the ones obtained from the fields $\Phi$ considered above. Not much details will be given, but the reader can check the result for the free massive fermion, (by Wick's theorem, the $n$-point functions of $\psi, \bar{\psi}$ are determinants of two-point functions, so it is sufficent to study the latter).

One would like to put

$$
\partial_{\lambda}\left\langle\phi_{1}\left(x_{1}\right) \cdots \phi_{n}\left(x_{n}\right)\right\rangle=\int d x\left\langle\Phi(x) \phi_{1}\left(x_{1}\right) \cdots \phi_{n}\left(x_{n}\right)\right\rangle,
$$

since one needs an expression which is linear in $\Phi$ and preserves translational invariance and locality. In general, however, the integral on the right hand side is divergent at the partial diagonals $x=x_{i}, i=1, \ldots, n$, and at infinity. We first regularise the divergence at infinity. One expects that the $x$-integration can be restricted to the domain $|x|<R$, when one componsates by adding a correction term

$$
\left\langle v(R) \mid \phi_{1}\left(x_{1}\right) \cdots \phi_{n}\left(x_{n}\right)\right\rangle
$$

to the right hand side of eq. (27). By the OPE expansion, $v(R)$ can be determined from the special case $n=1$. For 1-point functions we need

$$
\partial_{\lambda}\langle\phi(0)\rangle=0
$$

for all $\phi \in F$, which determines $v(R)$ uniquely. By eq. (16), no divergence at infinity occurs in $\int d x\langle\Phi(x) \phi(0)\rangle$, when the conformal dimensions of $\phi$ are sufficiently large. Thus we can restrict $v(R)$ to a finite dimensional subspace of $H^{*}$, when we take the limit $R \rightarrow \infty$. When $h(\Phi)+h(\phi)=1$ and $h(\phi)=\bar{h}(\phi)$, one has logarithmic divergences both at 0 and $\infty$, such that the choice of $v(R)$ must be correlated with the choice of the regularisation at the diagonal $x=0$, but this is no problem.

In the following we assume for ease of notation that the $x$-integration converges at infinity. To handle the other divergences, we exclude some $\epsilon$ neighbourhood of the partial diagonals and of infinity from the domain of integration and denote the integral over the complement of this domain by 
$\int_{\epsilon}$. For finite epsilon, locality is broken, so a limit $\epsilon \rightarrow 0$ is necessary, but to achieve convergence we need renormalisation.

To formulate perturbation theory in the presence of regularisations, we have to give a differential structure to the space of quantum field theories with a given vector space of fields $F$. Let $\mathbb{C}_{n}$ be the space $\mathbb{C}^{n}$ minus its partial diagonals. Let $\mathcal{F}_{n}$ be the space of functions $\mathbb{C}_{n} \times F^{n} \rightarrow \mathbb{C}$ which are linear in $F^{n}$, real analytic in $\mathbb{C}_{n}$ and have sufficiently good behaviour close to the partial diagonals and at infinity. A quantum field theory defines an element in $\mathcal{F}=$ $\oplus_{n} \mathcal{F}_{n}$. Suppose that the quantum field theory depends on some parameter space $\Pi$ and that the map $\Pi \rightarrow \mathcal{F}$ is differentiable. Perturbation theory is supposed to give the map $T \Pi \rightarrow T \mathcal{F}$ and its generalization to higher order jets.

This description is not quite right yet. The elements of $G L(F)$ act on $\mathcal{F}$ in a natural way. This does not lead to new quantum field theories, just to a reparametrisation of one and the same theory. Let $T_{0} \mathcal{F}$ be the subspace of $T \mathcal{F}$ given by the $G L(F)$ action. Then in general we only can expect that the map $T \Pi \rightarrow T \mathcal{F} / T_{0} \mathcal{F}$ is natural.

In the example of massive fermions, $\Pi$ is the positive real axis with parameter $\mu$, and the one-dimensional vector space $T \Pi$ is generated by $\Phi=: \bar{\psi} \psi: / 2$. More generally, it should be possible to identify $T \Pi$ with the real fields for which $h=\bar{h} \leq 1$. For a field $\Phi$ of this kind, we denote the corresponding element of $T \mathcal{F}$ at $f \in \mathcal{F}$ by $\partial_{\Phi} f$.

Let $P$ be the projection of $T \mathcal{F}$ to $T \mathcal{F} / T_{0} \mathcal{F}$. Then

$$
P \partial_{\Phi} \partial\left\langle\phi_{1}\left(x_{1}\right) \cdots \phi_{n}\left(x_{n}\right)\right\rangle=\lim _{\epsilon \rightarrow 0} P \int_{\epsilon} d x\left\langle\Phi(x) \phi_{1}\left(x_{1}\right) \cdots \phi_{n}\left(x_{n}\right)\right\rangle
$$

yields a natural candidate for a map $T L \rightarrow T \mathcal{F} / T_{0} \mathcal{F}$. Due to the OPE expansions for $\Phi \phi_{k}$ the right hand side is well defined. Indeed, the problems with convergence come from the most singular terms of the OPE, and the latter can be subtracted without changing the projection. In other words, one can find $\gamma(\epsilon) \in \operatorname{End}(F)$ such that

$$
\begin{aligned}
\lim _{\epsilon \rightarrow 0} \int_{\epsilon} d x & \left(\left\langle\Phi(x) \phi_{1}\left(x_{1}\right) \cdots \phi_{n}\left(x_{n}\right)\right\rangle\right. \\
& -\left\langle\left(\gamma(\epsilon) \phi_{1}\right)\left(x_{1}\right) \cdots \phi_{n}\left(x_{n}\right)\right\rangle-\ldots \\
& -\left\langle\phi_{1}\left(x_{1}\right) \cdots\left(\gamma(\epsilon) \phi_{n}\right)\left(x_{n}\right)\right)
\end{aligned}
$$

converges. The renormalisation just introduced is called wave function renormalisation. It only is sufficient for first order perturbation. For higher orders one has to take into account that the perturbing field $\Phi$ has to be renormalised itself. Higher order perturbation is non-unique, since one can perturb along arbitrary curves in $\Pi$. When we choose a flat connection on $T \Pi$, we can identify $\Pi$ itself with a subspace of $F$, at least locally, such that one can perturb along straight lines. Such a connection is called a renormalisation scheme. 
End of the digression, we now come to the Bethe ansatz. First we have to introduce the scattering matrices of integrable quantum field theories. The discussion will be very brief, a good pedagogical account is [D98]. In our treatment of conformally invariant theories in two space-time dimensions we first considered systems on a circle with circumference $L$. Because of scaling invariance, all values of $L$ are equivalent, so we put $L=2 \pi$. When a perturbation introduces a mass parameter $\mu$, the physics depends on the product $\mu L$. We are particularly interested in the scale invariant limit $\mu \rightarrow 0$ or equivalently $L \rightarrow 0$. Nevertheless, we also have to study the opposite limit $L \rightarrow \infty$, for which the space-time becomes $\mathbb{R}^{2}$. In this limit our systems is invariant under Lorentz transformations, which greatly simplifies the analysis. The scattering matrix is defined in this situation, which we will consider now.

The symmetry group of $\mathbb{R}^{2}$ with metric $d t^{2}-d x^{2}$ is the Poincaré group. The massive Dirac equation and the corresponding free field theory are invariant under this group, and we require invariance for all quantum field thories on $\mathbb{R}^{2}$. We have to consider the translations and the group of Lorentz transformations $S O(1,1)$, which is isomorphic to the additive group $\mathbb{R}$. The eigenvalues of the time and space translations are energy and momentum, which we denote by $(\omega, k)$. On irreducible representations of the Poincaré group, $\omega^{2}-k^{2}$ is constant. Because of locality, we do not want velocities $k / \omega$ which are greater than 1 (the velocity of light). Thus we need $\omega^{2}-k^{2}=m^{2} \geq 0$. We introduce a mass gap $\mu>0$, such that $m \geq \mu$ for all states occuring in the theory, apart from the vacuum. This is in contrast to CFTs which have many states with $m=0$. In our case we can parametrize energy-momentum as

$$
(\omega, k)=m(\cosh \theta, \sinh \theta) .
$$

Lorentz transformations act additively on $\theta$, such that the irreducible Poincaré representations are naturally isomorphic to the space of square integrable functions on the real line with parameter $\theta$. They are called one-particle spaces and interpreted as state spaces of a particle with mass $m$. When one has a conserved field $\phi$ with conformal spin $s$ such that $\bar{\partial} \phi=\partial \chi$, the action of the corresponding conserved quantitity on the state space is given by

$$
f(\theta) \rightarrow a \exp ((s-1) \theta) f(\theta),
$$

where the quantum number $a$ depends on the particle type. For the component $T$ of the energy-momentum tensor one has $s=2$ and a conserved quantity $\omega+k$, for $\bar{T}$ the conserved quantity is $\omega-k$. Thus one of the quantum number arising from conservations laws is the particle mass.

Since matter with non-zero mass cannot move at the velocity of light, and velocity now has a purely continuous spectrum, we expect that after a sufficiently long time any state of finite energy will separate into particles which move at different velocities. Thus at large times one can count the number of particles and determine their types. We assume that there is a finite number $r$ of particle types and index them by a set $I$. For each $i \in I$ 
we have a mass $m_{i}$ and a corresponding one-particle space $H_{i}^{1}$, with a natural isomorphism to $\mathcal{L}^{2}(\mathbb{R})$ described above.

Let

$$
H^{1}=\oplus_{i \in I} H_{i}^{1} .
$$

At large times, particles will be ordered according to their rapidities. Accordingly, let $T_{>}\left(H^{1}\right)$ be the subspace of the tensor algebra $T\left(H^{1}\right)$ for which the order of the rapidities corresponds to the order in the tensor products. It is more conventional to use symmetric and exterior products, depending on the statistics of the particles. For interacting particles in one space dimension the use of Bose or Fermi statistics is less natural than for interacting ones, however. One cannot exchange two particles without moving one through the other, so it is difficult to disentangle effects of statistics and interaction. Indeed, we will have to consider more general cases than Bose and Fermi statistics. In our context, statistics does not matter, since the partial diagonals of velocity space have measure 0 and $\Lambda H_{i}^{1}$ and $S H_{i}^{1}$ are isomorphic to $T_{>}\left(H^{1}\right)$.

In our description, the behaviour at large negative and large positive times yields isomorphisms

$$
H \simeq \otimes_{i \in I} T_{>}\left(H^{1}\right) .
$$

Combining the two isomorphisms yields a unitary transformation

$$
S: \otimes_{i \in I} T_{>}\left(H^{1}\right) \rightarrow \otimes_{i \in I} T_{>}\left(H^{1}\right),
$$

called the scattering matrix. States with 0 and 1 particles are invariant under $S$, so $S$ transforms multiparticle states to multiparticle states. In general, the scattering of two particles can produce arbitrarily many particles. Suppose, however, that one has a conserved field with conformal spin $s$. On states with momenta $\theta_{n}$ and particle types $i_{n} \in \mathrm{i}$ the eigenvalue of the corresponding conserved quantity is

$$
\sum_{n} a\left(i_{n}\right) \exp \left((s-1) \theta_{n}\right)
$$

and does not change by the scattering. Correspondingly, in integrable theories the number of particles, the rapidities $\theta_{n}$ and the quantum numbers $a\left(i_{n}\right)$ do not change in the scattering process.

In more than one space dimension integrable theories have to be free. Indeed, consider a situation where two particles pass each other at a large distance. Either they do not influence each other at all, in which case the theory is free, or one will see a small change in the direction of motion. When there is a single space dimension (i.e. two spacetime dimensions), many nonfree integrable models are known.

If two particle types $i, j$ of an integrable theory have identical quantum numbers $a(i)=a(j)$, in particular equal masses, scattering processes may transform one into the other. Theories of this kind are very interesting, but will not be considered here. We will assume that the conserved quantum numbers uniquely specify the particle type. 
In any quantum field theory, $n$-particle scattering will approximately factorize into a product of 2-particle scatterings, when at any given time at most two particles get close. In integrable theories this factorization is exact and remains true for all elements of $H$. Indeed, the symmetry operations given by the higher conserved quantities can be used to translate the particles by arbitrary distances. Thus the scattering matrix of an integrable theory with non-degenerate masses is determined by its restriction to

$$
S_{i j}: H_{i}^{1} \otimes H_{j}^{1} \rightarrow H_{j}^{1} \otimes H_{i}^{1},
$$

more precisely to the subspace with $\theta_{1}>\theta_{2}$. When the one-particle spaces are identified with $\mathcal{L}^{2}(\mathbb{R})$, the action of $S_{i j}$ is diagonal and given by a function $S_{i j}\left(\theta_{12}\right)$, with $\theta_{12}=\theta_{1}-\theta_{2}$. By unitarity, $\left|S_{i j}\left(\theta_{12}\right)\right|=1$. Instead of $\theta_{12}$, another natural variable is $\cosh \left(\theta_{12}\right)$, since $m_{1} m_{2} \cosh \left(\theta_{12}\right)=\omega_{1} \omega_{2}-k_{1} k_{2}$.

In terms of a Schrödinger wave function, a state with two particles of types $i, j$ with momenta $k_{1}, k_{2}$ and rapidities $\theta_{1}>\theta_{2}$ is described by

$$
\Psi\left(x_{1}, x_{2}\right)=\exp \left(i k_{1} x_{1}\right) \exp \left(i k_{2} x_{2}\right)
$$

for $x_{1} \ll x_{2}$ and by

$$
\Psi\left(x_{1}, x_{2}\right)=\exp \left(i \delta_{i j}\left(\theta_{12}\right) \exp \left(i k_{1} x_{1}\right) \exp \left(i k_{2} x_{2}\right)\right.
$$

for $x_{1} \gg x_{2}$. Exchanging the particle labelling yields

$$
\delta_{i j}(\theta)=-\delta_{j i}(-\theta) \quad \bmod \quad 2 \pi \mathbb{Z},
$$

at least for bosonic particles. For $\theta_{1}>\theta_{2}$ one has

$$
S_{i j}\left(\theta_{12}\right)=\exp \left(i \delta_{i j}\left(\theta_{12}\right)\right.
$$

since the region $x_{1} \ll x_{2}$ dominates for large negative and the region $x_{1} \gg x_{2}$ for large positive times. At finite time there is no singularity for $\theta_{1}=\theta_{2}$, so we expect $\delta_{i j}$ to be real analytic functions for all $\theta \in \mathbb{R}$. At fixed $\theta$, the value of $\delta_{i j}$ is only determined up to a multiple of $2 \pi$, but differences $\delta_{i j}(\theta)-\delta_{i j}\left(\theta^{\prime}\right)$ are uniquely defined real numbers, since $\delta_{i j}$ is continuous. We can fix $\delta_{i j}$ by a normalisation at infinite rapidity. Scattering at large rapidity difference probes the short distance behaviour of the interaction. At short distance, our $\delta>0$ perturbations become CFTs, for which there is no scattering. Thus we can put $\delta_{i j}(+\infty)=0$. Then eq. (29) yields

$$
\delta_{i j}(\theta)+\delta_{j i}(-\theta)+2 \pi A_{i j}=0,
$$

where $A$ is a symmetric $r \times r$ matrix with $A_{i j}=-\delta_{i j}(-\infty) /(2 \pi)$. From the preceding argument one expects $A_{i j} \in \mathbb{Z}$, but one can incorporate exotic statistics by admitting non-integral values.

For a given integrable theory, we can assume the particle spectrum and the scattering matrix to be known. In particular, the derivatives of the $\delta_{i j}$ are 
rational functions of $\cosh (\theta)$. In integrable theories which are invariant under space reflections, the scattering matrix elements have the form

$$
S_{i j}(\theta)=\prod_{x \in Q_{i j}} \frac{\sinh ((\theta+i \pi x) / 2)}{\sinh ((\theta-i \pi x) / 2)},
$$

with finite set $Q_{i j} \subset \mathbb{R}$. When the theory tends to a rational CFT at short distance, one even hast $Q_{i j} \subset \mathbb{Q}$. We will see that these $Q_{i j}$ determine in a direct way the matrix $A$ in (6) and thus the central object of our study. In principle the converse should be true, too. Indeed $A$ should characterize a CFT and an integrable perturbation which reproduces the $Q_{i j}$. It would be nice to find an algorithm which gives the result in a more direct way.

For integrable theories, systems of particles on a circle of circumference $L$ can be described by the Bethe ansatz. One extends the previous description of the Schrödinger wave function to small distances and looks at its phase change when one follows one particle position around the circle. Let us consider particles of types $i(1), i(2), \ldots$ with momenta $k_{1}, k_{2}, \ldots$. When the particles do not interact, each one can be described by a wave function $\exp \left(i k_{m} x\right)$. The $k_{m}$ are quantized in a simple way, since $k L$ must be an integral multiple of $2 \pi$, or a half-integral multiple for fermions in the NS case. When the phase changes are taken into account one finds

$$
k_{m} L+\sum_{n \neq m} \delta_{i(m) i(n)}\left(\theta_{m n}\right)=2 \pi N_{m},
$$

where in the bosonic case the $N_{m}$ must be integral. By eq. (30) the quantization of the total momentum is not affected by the interaction, as long as the $A_{i j}$ are integral.

For small $L$, the rapidities of right and left movers are of the order $\pm \log \left(2 \pi\left|N_{m}\right| / L\right)$, respectively. Thus for scattering processes between a left and a right mover the rapidity difference becomes infinite for $L \rightarrow 0$, whereas for two right- or two left-movers it becomes independent of $L$. Thus right movers and left movers decouple, except for statistical effects. For example, one may need a total fermion number which is even, or the right and left $N S$ and $R$ sectors may be coupled, as in the partition function (24).

We have seen that in one space dimension, bosons and fermions cannot be distinguished in the usual way, but a dynamical distinction is possible. For single $N S$ fermion states, the momentum should be a half-integral multiple of $2 \pi / L$. In this sense, one even can interpolated between bosonic and fermionic behaviour, by demanding that for a particle of type $i(m)$ one has

$$
N_{m} \equiv b_{i} \bmod \mathbb{Z},
$$

with arbitrary $b_{i}$. Values of $b_{i}$ which are neither integral nor half-integral imply exotic statistics. If such particles occur, one needs a balance between left and right to obtain integral total momentum (up to a factor $2 \pi / L$ ). To obtain a 
rational CFT in the $L \rightarrow 0$ limit, one needs of course $b_{i} \in \mathbb{Q}$. Similar remarks apply to $A_{i j}$.

It seems that for a given set of $N_{m}$, the $\theta_{i}$ are fixed uniquely. Nevertheless, the Bethe ansatz is incomplete, since the range of the $N_{m}$ is not specified. Let us assume that for particles of type $i$ all values $N \geq b_{i}$ are allowed. Then we can evaluate the partition function and its split into holomorphic and antiholomorphic characters in the CFT limit $L \rightarrow 0$. In this limit the interaction between left and right movers should vanish, since the rapidity difference for any pair of right and left movers is of order $-\log (\mu L)$. Among right movers, energy and momentum can be identified, so after rescaling the energy is given by $\sum_{m} k_{m} L / 2 \pi$, where the sum only extends over positive $k_{m}$. Thus the energy shift due to the interaction is given by

$$
-\sum_{n \neq m} \delta_{i(m), i(n)}\left(\theta_{m n}\right) / 2 \pi=\frac{1}{2} \sum_{i j} n_{i} A_{i j} n_{j}
$$

when there are $n_{i}$ particles of type $i$. Summing over the possibilities for the $N_{m}$ yields the form (6) for the corresponding character, up to a shift $h-c / 24$ of the ground state energy.

When we have Fermi statistics for particles of type $i$, such that $N_{m}, N_{k}$ have to be different when $m \neq k$ but $i=i(m)=i(k)$, a term $n_{i}\left(n_{i}-1\right) / 2$ is added to the energy, which can be absorved by a redefinition of $A, b$.

The matrix $A$ is given by the local interaction behaviour, but $b$ is a global quantity which can be different in different sectors of the theory. When $A_{i j} \notin \mathbb{Z}$ for some $i, j$ or $b_{i}-A_{i i} / 2 \notin \mathbb{Z}$ for some $i$, one gets a character which does not transform homogeneously under $\tau \mapsto \tau+2 \pi$. Nevertheless, in many cases one finds acceptable partition functions by averaging sums $\sum_{i j} n_{i j} \chi_{i} \bar{\chi}_{j}$ over the translations $\tau \mapsto \tau+2 \pi n, n \in \mathbb{Z}$. This averaging projects out states with exotic values of the total momentum. Thus eq. (6) has been explained, at least in an intuitive way.

\section{The connection to algebraic K-theory}

The preceding discussions indicate that the search for modular functions of the form

$$
\chi=\sum_{n \in \mathbb{N}^{r}} q^{n A n / 2+b n+h-c / 24}
$$

with a rational symmetric $r \times r$ matrix $A, h \in \mathbb{Q}$ and $b \in \mathbb{Q}^{r}$ will be very interesting. To assure convergence, we will assume that $A$ is positive.

The requirement that $\chi$ is modular imposes strong restrictions on $A, b, h$. In this article we wil not consider the restrictions on $b$. In any case, the CFTs under consideration have a unique $A$, whereas different representations of the OPE of $F_{h o l}$ yield sectors with different $b, h$. 
Recall that $q=\exp (2 \pi i / \tau)$ and $\tilde{q}=\exp (-2 \pi i / \tau)$. According to eq. (3), a modular character can be written as a sum over terms $\tilde{q}^{k}$, with real and rational $k$. Let us check how $\chi$ behaves at small $\tau$, where the dominant contribution should come from $k=-c_{e f f} / 24$.

For ease of notation we first consider the case $r=1$, but generalisation to arbitrary $r$ will be immediate. We have

$$
\chi=\oint_{C} \sum_{n \in Z} q^{n A n / 2+b n+h} x^{-n} \sum_{m \in \mathbb{N}^{r}} \frac{x^{m}}{(q)_{m}} \frac{d x}{2 \pi i x}
$$

where the path $C$ is a small circle around the origin. The first factor of the integrand can be evaluated by Poisson summation,

$$
\begin{aligned}
\sum_{n} & q^{n A n / 2+b n+h} x^{-n} \\
& =\sum_{m} \int_{n} \exp (2 \pi i(\tau(n A n / 2+b n+h)-n(u-2 \pi i m))) d n \\
& =(i \tau A)^{-1 / 2} \sum_{m} \exp \left(-\frac{(u-2 \pi i m) A^{-1}(u-2 \pi i m)}{4 \pi i \tau}+O\left(\tau^{0}\right)\right),
\end{aligned}
$$

where $u=\log x$. The integral over $x$ and the sum over $m$ can be combined into an integral over the simply connected cover of $C$, which yields an integral over $u$ along a parallel of the imaginary axis.

For the second factor one we have the explicit form

$$
\sum_{m \in \mathbb{N}} \frac{x^{m}}{(q)_{m}}=\prod_{n \in \mathbb{N}}\left(1-x q^{n}\right)^{-1}
$$

When $q$ is close to 1 , we can approximate the products over $n$ by integrals:

$$
\log \prod_{n \in \mathbb{N}}\left(1-x q^{n}\right)^{-1} \sim-\int_{0}^{\infty} d n \log \left(1-x q^{n}\right)=-L i_{2}(x) / 2 \pi i \tau .
$$

The integral over $u$ can be evaluated by the saddle point approximation. Vanishing of the derivative of

$$
\frac{u A^{-1} u}{2}+L i_{2}\left(x_{i}\right)
$$

yields

$$
A^{-1} u=v
$$

where $v=\log (1-x)$. Exponentiation yields

$$
x=(1-x)^{A} .
$$


Since $A$ is positive, the right hand side of this equation equals 1 for $x=0$ and 0 for $x=1$. The left hand side behaves in the opposite way, such that the equation has a real solution with $0<x<1$. Moreover, $x$ is algebraic and the corresponding Rogers dilogarithm $L(x)$ is demanded to be rational.

Altogether we obtain

$$
Z \sim \tilde{q}^{-k}
$$

where $k=L(x) /\left(4 \pi^{2}\right)$ and

$$
L(x)=\frac{u v}{2}+L i_{2}(x)
$$

is the Rogers dilogarithm. Since $k=c_{e f f} / 24$ we have

$$
c_{e f f}=\frac{L(x)}{L(1)} .
$$

Now $x$ is algebraic and $k$ must be rational. As explained in Zagier's talk, this happens for three cases only, namely $A=1, A=2$ or $A=1 / 2$. They correspond to $c_{\text {eff }}=1 / 2,2 / 5,3 / 5$, resp. All of them turn out to be realized in minimal models. Recall that $c=1-6(p-q)^{2} / p q$ for the $(p, q)$ model. The formula for $c_{\text {eff }}$ is similar, one has $c_{e f f}=1-6 / p q$. The models with $q-p=1$ are unitary. The cases $A=1$ and $A=2$ correspond to the free fermion and the $(2,5)$ minimal model and have been discussed in detail in section 2 . The case $A=1 / 2$ yields the $(3,5)$ minimal model with $c_{\text {eff }}=3 / 5$.

Now let us consider higher $r$. We have

$$
\chi=\oint_{C} \sum_{n \in Z} q^{n A n / 2+b n+h} X^{-n} \sum_{m \in \mathbb{N}^{r}} \frac{X^{m}}{(q)_{m}} \prod_{i=1}^{r} \frac{d x_{i}}{2 \pi i x_{i}},
$$

where the path $C$ now is a Cartesian product of small circles around the origin. We use the notation $X^{m}=\prod_{i} x_{i}^{m_{i}}$ and analogously for $X^{-n}$. As before, Poisson summation of the first factor yields

$$
\chi \sim \int \exp \left(-\frac{U A^{-1} U / 2+\sum_{i} L i_{2}\left(x_{i}\right)}{2 \pi i \tau}\right) \prod_{i=1}^{r} d u_{i},
$$

where $U=\left(u_{1}, \ldots, u_{r}\right)$ and $x_{i}=\exp \left(u_{i}\right)$.

When we apply the saddle point method, vanishing of the derivatives of $U A^{-1} U / 2+\sum_{i} L i_{2}\left(x_{i}\right)$ yields

$$
A^{-1} U=V,
$$

where $V=\left(v_{1}, \ldots, v_{r}\right)$ and $\exp \left(v_{i}\right)+\exp \left(u_{i}\right)=1$.

The Riemann surface

$$
\hat{\mathbb{C}}=\{(u, v) \in \mathbb{C} \mid \exp (u)+\exp (v)=1\}
$$


already appeared in Zagier's talk. We complete $\hat{\mathbb{C}}$ at $u=0$ and $v=0$ by adjoining the points $(0, \infty)$ and, which will be useful for bookkeeping. Values of functions at these points will be assigned when the functions have a unique limit.

When the intergration path for $Z$ is deformed, several saddle points may appear. They all have to satisfy the equation $U=A V,(U, V) \in \mathbb{C}^{r}$. The corresponding contribution to $Z$ is proportional to $\tilde{q}^{-k}$, where up to an integral summand

$$
k=\sum_{i=1}^{r} L\left(u_{i}, v_{i}\right) /\left(4 \pi^{2}\right)
$$

Here the function

$$
L: S \rightarrow \mathbb{C} / \mathbb{Z}(2)
$$

is the analytic continuation of Rogers dilogarithm discussed by Zagier. It can be characterized by $L(\infty, 0)=0$ and

$$
d L=(u d v-v d u) / 2 .
$$

and has the properties

$$
\begin{aligned}
L(0, \infty) & =\frac{\pi^{2}}{6} \\
L(u+2 \pi i, v) & =L(u, v)+\pi i v \\
L(u, v+2 \pi i u) & =L(u, v)-\pi i u .
\end{aligned}
$$

The multivaluedness of $L$ arises from the residues of $d(L+u v / 2)=u d v$. Since $d v=d u /(\exp (u)-1)$, these residues are multiples of $2 \pi i$. The notation $\mathbb{Z}(2)$ stands for $(2 \pi i)^{2} \mathbb{Z}$ and serves as a reminder the the proper context should be the theory of motives.

Let $[\hat{\mathbb{C}}]$ be the free abelian group with basis $[(u, v)]$ for $(u, v) \in \hat{\mathbb{C}}$. We extend $L$ to a linear function on $[\hat{\mathbb{C}}]$. Elements of $[\hat{\mathbb{C}}]$ will be denoted by $(U, V)$. The effective central charge given by the solutions of out equation $U=A V$ is

$$
c_{e f f}=\frac{6}{\pi^{2}} L(U, V) \quad \bmod \quad 24 \mathbb{Z}
$$

for the dominant saddle point $(U, V)$, whereas the other saddle points yield

$$
c-24 h_{i}=\frac{6}{\pi^{2}} L(U, V) \bmod \quad 24 \mathbb{Z}
$$

for other conformal weights $h_{i}$ of the CFT. F There is an infinite number of solutions of $U=A V$, and we have to consider the corresponding values of $L(U, V)$. After exponentiating the equation we obtain the algebraic equations

$$
x_{i}=\prod_{j=1}^{r}\left(1-x_{j}\right)^{A_{i j}} .
$$


As long as these equations are independent, they only yield a finite number of solutions. Solutions of $U=A V$ which yield the same $x_{i}$ are related by

$$
\begin{aligned}
& U^{\prime}=U+2 \pi i m \\
& V^{\prime}=V+2 \pi i n,
\end{aligned}
$$

$m, n \in \mathbb{Z}^{r}$, where $m=A n$. We have

$$
L\left(U^{\prime}, V^{\prime}\right)=L(U, V)+\pi i(m V-n U)+2 \pi^{2} m n .
$$

Since $A$ is symmetric, $m V-n U=n A^{t} V-n A V=0$. We call $A$ even whenever $m=A n, m, n \in \mathbb{Z}^{r}$ implies that $m n$ is even. In this case

$$
L\left(U^{\prime}, V^{\prime}\right)=L(U, V) \bmod \quad Z(2) .
$$

In general we have $2 L\left(U^{\prime}, V^{\prime}\right)=2 L(U, V) \bmod \quad Z(2)$. F Now we will introduce the extended Bloch group $\hat{B}(\mathbb{C})$ as subquotient of $[\hat{\mathbb{C}}]$ N93, N03. On the latter group we have a natural linear map $\sigma:[\hat{\mathbb{C}}] \rightarrow \mathbb{C} \otimes_{\mathbb{Z}} \mathbb{C}$ induced by

$$
\sigma(u, v)=u \otimes_{\mathbb{Z}} v-v \otimes_{\mathbb{Z}} u,
$$

$\sigma(0, \infty)=\sigma(\infty, 0)=0$. Let $\mathcal{P}$ be the kernel of this map. We define

$$
\hat{B}(\mathbb{C})=\mathcal{P} / \mathcal{P}_{0},
$$

where the subgroup $\mathcal{P}_{0}$ of $\mathcal{P}$ is generated by all elements of the forms

$$
\begin{array}{r}
(u, v)+(v, u)-(0, \infty) \\
(u-2 \pi i, v)+2(u-v-\pi i,-v)+(u, v) \\
\sum_{i=1}^{5}\left(u_{i}, v_{i}\right)-2(0, \infty),
\end{array}
$$

where in the last line

$$
u_{i}=v_{i-1}+v_{i+1} \quad \text { for } \quad i=1, \ldots 5
$$

and $v_{0}=v_{5}, v_{1}=v_{6}$ for cyclic symmetry. The first two lines correspond to $[x]+[1-x]=0$ and $2[1-x]+2\left[1-x^{-1}\right]=0$ in $B(\mathbb{C})$, the last to the five-term relation. The elements in $\mathcal{P}_{0}$ are all annihilated by $L$, as one can prove easily by differentiation. In particular, the five-term identity asserts

$$
\sum_{i=1}^{5} L\left(u_{i}, v_{i}\right)=\pi^{2} / 3 .
$$

Thus we can consider $L$ as a map $L: \hat{B}(\mathbb{C}) \rightarrow Z / Z(2)$. Note that the obvious involution of $\mathcal{P}$ which is induced by $(u, v) \mapsto(v, u)$ is not an involution of $\mathcal{P}_{0}$. Instead we have $L(u, v)=L(0, \infty)-L(v, u)$. 
The Bloch group is of relevance for us, since due to the symmetry of $A$ all solutions of $U=A V$ yield elements $(U, V) \in \mathcal{P}$. Since $\hat{B}(\mathbb{C})$ is less well understood that $B(\mathbb{C})$ we want to relate it to the latter group. We have seen that there is a map $\hat{B}(\mathbb{C}) \rightarrow B(\mathbb{C})$ given by $(U, V) \mapsto \sum_{i}\left[x_{i}\right]$, where $U=\left(u_{1}, u_{2}, \ldots\right)$ and $x_{i}=\exp \left(u_{i}\right)$. We will see that this map is surjective up to torsion, i.e. the cokernel of the map contains only elements of finite order.

Let $\sum_{i}\left[x_{i}\right] \in B(\mathbb{C})$ and $y_{i}=1-x_{i}$. Then the element $\sum_{i} x_{i} \times y_{i} \in\left[\mathbb{C}^{*} \times \mathbb{C}^{*}\right]$ must lie in the subgroup generated by elements of the form $\left[z_{m} \times z_{n}\right]+\left[z_{n} \times z_{m}\right]$ and $\sum_{m} r_{m}\left[z_{m} \times z_{n}\right]$, where $\prod_{m} z_{m}^{r_{m}}=1$. Thus

$$
\sum_{i}\left[x_{i} \times y_{i}\right]=\sum_{m, n} C_{m n}\left[z_{m} \times z_{n}\right]
$$

where

$$
C_{m n}=R_{m k} F_{k n}+G_{m n}
$$

$R, F, G$ are integral matrices, $G$ is even and for all $k$

$$
\prod_{m} z_{m}^{R_{m k}}=1
$$

Note that the $x_{i}, y_{i}$ must be among the $z_{m}$. Now we choose logarithms $w_{m}$ of the $z_{m}$, inlcuding $u_{i}$ for $x_{i}$ and $v_{i}$ for $y_{i}$. Thus

$$
\sum_{m} R_{m k} w_{m}=2 \pi i q_{k}
$$

for all $k$ and integral $q_{k}$. By choosing different logarithms, we can change the vectors $\left(q_{k}\right)$ by integral linear combinations of the $\left(R_{m k}\right), m=1,2, \ldots$ We have

$$
N \sum_{i} \sigma\left[u_{i} \times v_{i}\right]=\sum_{k n} N q_{k} F_{k n} \sigma\left[2 \pi i \times w_{n}\right]
$$

for $N \in \mathbb{N}$. After choosing $N$ such that $\left(N q_{k}\right)$ is a linear combinations of the $\left(R_{m k}\right)$, one can change the choice of the logarithms, in general in different ways for the $N$ copies, such that the r.h.s. vanishes. Thus $N\left[x_{i}\right]$ lies in the image of $\hat{B}(\mathbb{C})$ in $B(\mathbb{C})$.

The linear map on $[\hat{\mathbb{C}}]$ induced by $(u, v) \mapsto(u \bar{v}-v \bar{u})$ factors through the wedge product. Thus $\mathcal{P}$ is contained in its kernel. The value $D(\exp (u))$ of the Bloch-Wigner function $D$ discussed in Zagier's talk is the imaginary part of $L(u, v)-(u \bar{v}-v \bar{u}) / 4$. Thus on $\hat{B}(\mathbb{C})$ the imaginary part of $L$ coincides with $D$, but the real part yields new information. When $\Im L(U, V) \neq 0$, then $L(U, V)$ and consequently $(U, V)$ have infinite order. For $B(\mathbb{C})$ there is a converse to this statement. Since $B(\mathbb{C})=B(\bar{Q})$ we can restrict ourselves to algebraic numbers. When $\sum_{i} n_{i}\left[x_{i}\right] \in B(K)$ for some algebraic number field $K$ and $D$ yields 0 for this element and all its Galois conjugates, then the element is of finite order, see [DZ91], section 2. Presumably this also is true for preimages of such elements in $\hat{B}(\mathbb{C})$. 
Now we consider our saddle points $(U, V)$ with $U=A V$. Since $A$ is symmetric, we have $\sum_{k} u_{k} \wedge v_{k}=0$, such that $(U, V) \in \mathcal{P}$. Moreover, the modular character $\chi$ must be a sum rational powers of $\tilde{q}=\exp (-2 \pi i / \tau)$. In particular this means that $L(U, V) /\left(4 \pi^{2}\right)$ is rational or equivalently that $L(U, V)$ is an element of finite order in $\mathbb{Q} / \mathbb{Z}(2)$. This is true for all solutions of $U=A V$, including those obtained by Galois conjugation of the solutions of the corresponding exponentiated equation. Thus we know that $(U, V)$ maps to 0 in $B(\mathbb{C})$, and we expect that it is an element of finite order in $\hat{B}(\mathbb{C})$. Obviously, the argument is incomplete, but one can certainly expect to find a real proof along these lines.

Over the real numbers it is known that the any torsion element of the Bloch group can be represented by a real and rational linear combination of roots of unity [FS93]. The latter form the field $\mathbb{Q}_{a b}^{+}$. Thus it is a plausible conjecture that for any symmetric matrix $A$ such that $U=A V$ has discrete solutions which are torsion elements of the Bloch group the corresponding algebraic numbers $\exp \left(u_{k}\right)$ belong to $\mathbb{Q}_{a b}^{+}$.

Work on statistical models and related integrable theories has produced a partly conjectural list of matrices $A$ belonging to this class which are related to the Dynkin diagrams of type ADET. These diagrams $A_{r}, D_{r}, E_{r}, T_{r}$ have $r$ vertices, with $r \geq 4$ for $D_{r}$ and $r=6,7,8$ for $E_{r}$. Recall that $r$ is called the rank of $X_{r}$ and that the corresponding Cartan matrix $\mathcal{C}\left(X_{r}\right)$ has off-diagonal entries $\mathcal{C}\left(X_{r}\right)_{i j}$ which are equal to -1 when the vertices $i, j$ are linked by an edge of the diagram and equal to 0 when they are not. Since the ADE diagrams have no loops, one has $\mathcal{C}\left(X_{r}\right)_{i i}=2$ for $i=1, \ldots, r$. The Dynkin diagrams of type $A$ are just rows of vertices with linked neighbours, such that $\mathcal{C}\left(X_{r}\right)_{i j}=-1$ for $|i-j|=1$ and $\mathcal{C}\left(X_{r}\right)_{i j}=0$ for $|i-j|>1$. The tadpole diagram $T_{r}$ is obtained by folding $A_{2 r}$ diagrams in the middle, such that one gets a pairwise identification of the vertices. One has $\mathcal{C}\left(T_{r}\right)_{r r}=1$, otherwise the matrix elements of $\mathcal{C}\left(T_{r}\right)$ and $\mathcal{C}\left(A_{r}\right)$ are the same. Note that $\mathcal{C}\left(A_{1}\right)=(2)$ and $\mathcal{C}\left(T_{1}\right)=(1)$. We need positive definite Cartan matrices, which excludes $E_{r}$ for $r>8$. Indeed, $\operatorname{det}\left(A_{r}\right)=r+1, \operatorname{det}\left(D_{r}\right)=4, \operatorname{det}\left(E_{r}\right)=9-r$ and $\operatorname{det}\left(T_{r}\right)=1$. We also need the Coxeter numbers for these diagrams, which are $h\left(A_{r}\right)=r+1, h\left(D_{r}\right)=2 r-2, h\left(E_{6}\right)=12, h\left(E_{7}\right)=18, h\left(E_{8}\right)=30$, $h\left(T_{r}\right)=h\left(A_{2 r}\right)=2 r+1$.

From pairs of ADET Dynkin diagrams $X, Y$ one obtains the matrices

$$
A(X, Y)=\mathcal{C}(X) \otimes \mathcal{C}(Y)^{-1} .
$$

When $U=A(X, Y) V$ it is known or conjectured that $(U, V)$ is a torsion element of $\hat{B}(\mathbb{C})$. Let us check this in the simplest examples. For rank 1 we have the pairs $\left(A_{1}, A_{1}\right),\left(T_{1}, T_{1}\right),\left(A_{1}, T_{1}\right)$ and $\left(T_{1}, A_{1}\right)$. The yield $A=(1),(2),(1 / 2)$ in agreement with our examples from minimal models. The general formula for the effective central charge is known or conjectured to be

$$
c_{e f f}(X, Y)=\frac{r(X) r(Y) h(X)}{h(X)+h(Y)} .
$$


This agrees with the previous results for the rank 1 cases. Indeed, for $A=1$, the equation $x=(1-x)^{A}$ yields $x=1 / 2$. Since $[x]+[1-x]=0$ in $B(\mathbb{C})$, we have $2[1 / 2]=0$. In $\hat{B}(\mathbb{C})$ we can impose $u=v$, $\exp (u)=1 / 2$, which yields $(u, u)+(u, u)=(0, \infty)$ and $L(u, u)=\pi^{2} / 12$. For $A=2$ the equation $x=(1-x)^{A}$ yields the golden ratio. With $u=2 v$ we obtain $5(u, v)=2(0, \infty)$ in $\hat{B}(\mathbb{C})$ and $L(u, v)=\pi^{2} / 15$.

For $A=1 / 2$ we consider more generally $A(X, Y)=A(Y, X)^{-1}$. In special cases this corresponds to level-rank duality. More generally, replacement of $A$ by $A^{-1}$ just yields an exchange of $U$ and $V$ in the solutions of $U=A V$, which is the involution mentioned above. Note that for an even matrix $A$ its inverse is even, too. Moreover $L(u, v)+L(v, u)=L(0, \infty)$ yields $L(U, V)+L(V, U)=$ $\operatorname{rank}(A) L(0, \infty)$, which agrees with

$$
c_{e f f}(X, Y)+c_{e f f}(Y, X)=r(X) r(Y) .
$$

For $X=A_{k-1}$ and $Y$ of $A D E$ type, comparison of eq. (32) yields with eqs. (20) and (21) yields

$$
\begin{gathered}
c_{e f f}\left(A_{k-1}, Y\right)=\tilde{c}(Y, k) \\
c_{e f f}\left(Y, A_{k-1}\right)=\check{c}(Y, k) .
\end{gathered}
$$

The theories with field spaces $\tilde{F}_{Y}^{k}$ and $\check{F}_{Y}^{k}$ are unitary, so this is an equality between two effective central charges. For the special case $k=1, Y=A_{1}$ one knows that the theory has characters of the form (6) with $A=A\left(A_{1}, A_{1}\right)=1$ and for many other cases there is good numerical or analytical evidence that it is true, too K87, KN92, KM93, so it probably is true in general. This would yield equalities relating the dilogariths of the corresponding solutions of $U=A V$ with the conformal dimensions of these theories, and conversely these equalities would provide good evidence for the conjecture. In the following section we shall make a first step by finding all solutions of $U=A V$ for $A=A\left(A_{m}, A_{n}\right)$ and general $m, n$.

There is a way to prove that $A(X, Y)$ yields torsion elements for any specified pair $X, Y$, though it has to be applied separately for each such pair and does not give a general proof Z91, GT95. We give a slightly modified version of the method. First note that $U=A V$ is equivalent to

$$
(\mathcal{C}(Y) \otimes I) U=(I \otimes \mathcal{C}(X)) V
$$

Let $C$ be the Cartan matrix of $A_{2(h(X)+h(Y))}^{(1)}$, the Dynkin diagram of which is a regular polygon. Consider the equation

$$
(\mathcal{C}(Y) \otimes I \otimes I-I \otimes I \otimes C) U^{\prime}=(I \otimes \mathcal{C}(X) \otimes I-I \otimes I \otimes C) V^{\prime}
$$

with

$$
U^{\prime}, V^{\prime} \in \mathbb{C}^{r(Y)} \otimes \mathbb{C}^{r(X)} \otimes \mathbb{C}^{P}
$$


and $P=2(h(X)+h(Y))$. With respect to the last factor we write $U^{\prime}=$ $\left(U^{1}, \ldots, U^{P}\right)$ and analogously for $V^{\prime}$. One easily sees that Zamolodchikov's equation determines $U^{n+1}$ in terms of $U^{n-1}, U^{n}$. Zamolodchikov now claims that $U^{1}, U^{2}$ can be chosen arbitrarily. In other words, the pair $U^{P-1}, U^{P}$ yields $U^{1}$ again, such that the solution is periodic in $n$ with period $P$. For the half period $P / 2$ one has

$$
U_{i j}^{n+P / 2}=U_{\sigma(i) \sigma(j)}^{n},
$$

where $\sigma$ acts as the involutive diagram symmetry on the vertices of $A_{r}, D_{2 r+1}$, $E_{6}$, and trivially for the other ADET diagrams. This claim is true for every pair $X, Y$ checked so far, but a general proof is lacking. Whenever it is true one obtains continuous families of elements $\left(U^{\prime}, V^{\prime}\right) \in \hat{B}(\mathbb{C})$ and no component needs to have an algebraic exponential. This implies $\left(U^{\prime}, V^{\prime}\right)=0$. On the other hand we have a special solution

$$
\left(U^{\prime}, V^{\prime}\right)=(\underbrace{U, \ldots U}_{P \text { times }}, \underbrace{V, \ldots, V}_{P \text { times }}),
$$

whenever $U=A V$. Thus $\left(U^{\prime}, V^{\prime}\right)=P(U, V)$ and $(U, V)$ is an element of finite order in $\hat{B}(\mathbb{C})$, with an order dividing $P$. When $\sigma$ acts trivially on $X$ and $Y$, the order is even a factor of $P / 2$. In particular, the products $P c_{e f f}$ and $24 P(h(X)+h(Y)) h_{i}$ should be even integers when $\sigma$ acts trivially, and integral in any case.

\section{Solving the algebraic equations in special cases}

Let us consider the equation $U=A V$ for special cases of $A=A(X, Y)$. We will be able to find all solutions for $X=A_{1}$ and also for $A\left(A_{m}, A_{n}\right)$, with arbitrary $m, n$. Finding the solutions needs methods from Lie algebra theory, but we shall try first, how far one can get by elementary algebra alone. This will be sufficient for $X=A_{1}$, and it will make some of the later developments easier to understand.

We first rewrite $U=A V$ as $\mathcal{C}(Y) U=\mathcal{C}(X) V$. In order have a chance to find logarithms of algebraic integers we put $U=-\mathcal{C}(X) W$. With $Z=\exp (W)$ we obtain

$$
Z^{2-\mathcal{C}(X)}+Z^{2-\mathcal{C}(Y)}=Z^{2}
$$

In this form all exponents are positive integers and all coefficients are 1 , so we have a good chane to find algebraic integers. This equation has many solutions for which some components of $Z$ vanish. Those will be called non-admissible, since they do not yield solutions of $U=A V$. When one treats the equations by elementary algebra it may be useful to find them first, however, since this reduces the degree of the $Z$ equations. Many of the following considerations can be regarded as a systematic procedure to eliminate the non-admissible solutions. 
In general $Z$ has components $z_{i j}$, where $i$ labels the vertices of the Dynkin diagram of $X$ and $j$ those of $Y$. We first consider the case $X=A_{1}$ where the first index is superfluous. Thus for $Y=A_{n}$ we have variables $Z=\left(z_{1}, \ldots, z_{n}\right)$. One equation links $z_{1}, z_{2}$, the next ones link $z_{1}, z_{2}, z_{3}$, then $z_{2}, z_{3}, z_{4}$ and so on. To find a uniform solution we use the semi-infinite $n \rightarrow \infty$ limit of $A_{n}$ and an infinite series of variables $Z=\left(z_{1}, z_{2}, \ldots\right)$. Generically every equation determines $z_{n}$ in terms of the preceding $z_{k}$, thus eventually in terms of $z_{1}$. To reduce to the case $Y=A_{n}$ we just have to take the first $n$ equations and to put $z_{n+1}=1$. The $D$ and $E$ cases can be handled similarly, though the branching needs extra attention. For the resulting values of $c_{\text {eff }}$ see [KM90. take the first $n$ equations and to put $z_{n+1}=1$. The $D$ and $E$ cases can be handled similarly, though the branching needs extra attention.

For the $A$ series the equation are

$$
1+z_{i-1} z_{i+1}=z_{i}^{2},
$$

$i=1,2, \ldots$, where we put $z_{0}=1$. The equations are invariant under sign change of the $z_{i}$. When $z_{m}=0$, the equations for $z_{k}$ with $k<m$ and $k>m$ decouple from each other. We have $z_{m-1}=1$ and $z_{m+1}=1$, both up to a sign. But let us concentrate on the admissible case where no $z_{i}$ vanishes. Then a priori the $z_{i}$ become rational functions of $z_{1}$.

The reader who does not know this recursion will find it somewhat miraculous that the $z_{i}$ turn out to be polynomials in $z_{1}$ with integer ocefficients. Indeed $z_{i}=p_{i}(z)$, where the $p_{i}$ are the standard Chebysheff polynomials and we have put $z_{1}=z$. These polynomials are defined by a linear recursion, namely

$$
p_{i+1}(z)+p_{i-1}(z)=z p_{i}(z)
$$

with $p_{0}(z)=1$ and $p_{1}(z)=z$. By they satisfy the quadratic recursion required for the $z_{i}$. Indeed

$$
\begin{aligned}
1+p_{i-1} p_{i+1}=1+\left(z p_{i}-p_{i-1}\right) p_{i-1} & = \\
1-p_{i-1}^{2}+p_{i-2} p_{i}+\left(z p_{i-1}-p_{i-2}\right) p_{i} & =p_{i}^{2} .
\end{aligned}
$$

For $z=\omega+\omega^{-1}$ one easily obtains by induction from (33)

$$
p_{i}(z)=\omega^{i}+\omega^{i-2}+\ldots+\omega^{-i}
$$

thus

$$
p_{i}(z)=\frac{\omega^{i+1}-\omega^{-i-1}}{\omega-\omega^{-1}}
$$

for $\omega \neq 1,-1$.

For later use we will define polynomials $p_{i}(z)$ for negative $i$ by extending the linear recursion relation to such negative values. This is easy, since the recursion relation is invariant under a sign change of $i$. We obtain $p_{-1}(z)=0$, $p_{-2}(z)=-1$ and more generally $p_{-i}(z)=-p_{i-2}(z)$. Note that 2 is the Coxeter number of $A_{1}$. 
The solution of $U=A V$ for $A\left(A_{1}, A_{n}\right)$ can be obtained by specializing the previous result to $p_{n+1}(z)=1$. This yields $p_{n}(z) p_{n+2}(z)=0$. The case $p_{n}(z)=0$ is not admissible. Thus we obtain

$$
\omega^{2(n+3)}=1,
$$

excluding $\omega^{2}=1$. This is the expected result, since

$$
h\left(A_{1}\right)+h\left(A_{n}\right)=n+3 .
$$

Let us denote the resulting value of $p_{i}(z)$ by $z_{i}$. Since $z_{n+1}=1$ and $z_{n+2}=0$, the linear recursion yields $z_{n+3}=-1$ and more generally $z_{i+n+3}=-z_{i}$. Thus we have an anti-periodic behaviour with period $n+3$, which is the sum of the Coxeter numbers of $A_{1}$ and $A_{n}$. This behaviour generalizes to all ADET Lie algebras. Together with $p_{-i}(z)=-p_{i-2}(z)$ it yields $z_{i}=z_{n+1-i}$.

Now consider $A\left(A_{1}, D_{n+2}\right)$. We write $Z=\left(z_{1}, z_{2}, \ldots, z_{n}, z^{\prime}, z^{\prime \prime}\right)$, where $z^{\prime}, z^{\prime \prime}$ are the variables for the vertices on the short arms of the Dynkin diagram of $D_{n+2}$. One obtains the equations

$$
\begin{aligned}
1+z_{i-1} z_{i+1} & =z_{i}^{2} \quad \text { for } \quad i=1,2, \ldots, n-1 \\
1+z_{n-1} z^{\prime} z^{\prime \prime} & =z_{n}^{2} \\
z^{\prime 2}=z^{\prime \prime 2} & =1+z_{n}
\end{aligned}
$$

This yields $z_{k}=p_{k}(z), z^{\prime} z^{\prime \prime}=p_{n+1}(z)$ and $z^{\prime \prime}= \pm z^{\prime}$. The equations $1+$ $p_{n}(z)= \pm p_{n+1}(z)$ are of degree $n+1$ and all solutions are found easily when we put $z=\omega+\omega^{-1}$ as before. Solutions are obtained from $\omega= \pm 1$ or $\omega^{n+1}= \pm 1$ or $\omega^{n+2}=1$. The latter two possibilities are not admissible, however, since they lead to $z_{n}=0$ or to $z^{\prime}=z^{\prime \prime}=0$. For $\omega= \pm 1$ the choice of sign is irrelevant. One obtains

$$
\exp \left(u_{k}\right)=(k+1)^{-2}
$$

for $k=1, \ldots, n$ and $\exp \left(u^{\prime}\right)=\exp \left(u^{\prime \prime}\right)=(n+2)^{-1}$.

The corresponding central charge is $c_{e f f}=1$, independently of $n$. This follows easily by induction in $n$ and insertion of $2 u=u_{k}$ in the doubling formula

$$
2 L(u, v)=2 L\left(u-v^{\prime}, v^{\prime}\right)+L\left(2 u, v+v^{\prime}\right)
$$

valid for $\exp \left(v^{\prime}\right)=\exp (u)+1$. The doubling formula is obtained from eq. (31) by the identification $v_{2}=v_{4}=u$ and use of $L(u, v)+L(v, u)=\pi^{2} / 6$.

The case $A\left(A_{1}, E_{n+3}\right)$ is a bit more complicated. We use variables $z_{1}, \ldots, z_{n}$ for the longest branch, plus $u_{1}, u_{2}, t$ for the short branches. We put $u=u_{1}$, $z=z_{1}$. The variable $t$ is determined by $t^{2}=1+z_{n}$. When none of the variables vanishes, we have $z_{k}=p_{k}(z)$, but also $p_{3}(u)=u^{3}-2 u=p_{n}(z)$ and

$$
t u_{2}=t\left(u^{2}-1\right)=p_{n+1}(z) .
$$

Eliminating $t, u$ is now easy. The resulting polynomial equation for $z$ has many unacceptable solutions for which some $p_{k}(z), k=1, \ldots, n+1$ vanishes. The remaining solutions are given for $n=3$ by 


$$
z^{3}-2 z^{2}-z+1=0
$$

thus $z=\omega+\omega^{-1}+1$ with $\omega^{7}=1$, for $n=4$ by

$$
\left(z^{2}-3\right)^{2}=5
$$

thus $z=\sqrt{2}(\sqrt{5}+1) / 2$ up to sign choices, and for $n=5$ by

$$
(z-1)^{2}=2 .
$$

As next case let us consider $A=A\left(T_{1}, A_{\infty}\right)$, with $A_{\infty}$ as before. This time we obtain the recursion relation

$$
z_{i}+z_{i-1} z_{i+1}=z_{i}^{2}
$$

We put $z_{k}=q_{k}(z)$. The miracle repeats, with new polynomials. They satisfy the recursion relation

$$
q_{i+1}(z)=z q_{i}(z)-q_{i}(z)-q_{i-1}(z)+1
$$

With $z=\omega+1+\omega^{-1}$ we find

$$
z_{i}=\frac{\left(\omega^{(i+2) / 2}-\omega^{-(i+2) / 2}\right)\left(\omega^{(i+1) / 2}-\omega^{-(i+1) / 2}\right)}{\left(\omega-\omega^{-1}\right)\left(\omega^{1 / 2}-\omega^{-1 / 2}\right)}
$$

where a limit has to be taken for $\omega^{2}=1$. For $A=A\left(T_{1}, A_{n}\right)$ we have to impose $z_{n+1}=1, z_{n+2}=0$ as before. This yields

$$
\omega^{n+4}=1 \text {. }
$$

Moreover, only primitive $(n+4)$-th roots of unity are admissible, since otherwise $z_{i}=0$ for some $i \leq n$.

Now let us consider $A=A\left(A_{2}, A_{\infty}\right)$. We write $z_{1 i}=x_{i}, z_{2 i}=y_{i}$ and obtain the equations

$$
\begin{aligned}
x_{i}^{2} & =y_{i}+x_{i-1} x_{i+1} \\
y_{i}^{2} & =x_{i}+y_{i-1} y_{i+1}
\end{aligned}
$$

for $i=0,1, \ldots$, with $x_{0}=y_{0}=1$ and $x_{i}=y_{i}=0$ for $i<0$. The miracle repeats and we find

$$
\begin{aligned}
& x_{i+1}=x x_{i}-y x_{i-1}+x_{i-2} \\
& y_{i+1}=y y_{i}-x y_{i-1}+y_{i-2}
\end{aligned}
$$

for $i=0,1, \ldots$, with $x=x_{1}, y=y_{1}$.

For $x_{i}=y_{i}$ the equations reduce to the case $A\left(T_{1}, A_{\infty}\right)$. To prove the result in general we use the additive recursion as definition of the $x_{i}, y_{i}$ and 
prove the quadratic recursion formula. The latter is true for $i=-1,0,1$. For $i \geq 1$ we have

$$
\begin{aligned}
x_{i+1}^{2}-x_{i} x_{i+2} & =x_{i+1}\left(x x_{i}-y x_{i-1}+x_{i-2}\right)-x_{i}\left(x x_{i+1}-y x_{i}+x_{i-1}\right) \\
& =y\left(x_{i}^{2}-x_{i-1} x_{i+1}\right)-x_{i} x_{i-1}+x_{i+1} x_{i-2} \\
& =y y_{i}-x\left(x_{i-1}^{2}-x_{i} x_{i-2}\right)+x_{i-2}^{2}-x_{i-3} x_{i-1} \\
& =y y_{i}-x y_{i-1}+y_{i-2}=y_{i+1} .
\end{aligned}
$$

Interchange of $x, y$, i.e. the symmetry of $A_{2}$ yields the other quadratic recursion formula.

We can use the linear recursion relation to define $x_{i}, y_{i}$ for negative values of $i$. We have

$$
x_{i-2}=y x_{i-1}-x x_{i}+x_{i+1}
$$

This yields $x_{i}=y_{i}=0$ for $i=-1,-2$ and $x_{-i}=y_{i-3}$. When $x_{n+1}=y_{n+1}=1$ and $x_{n+2}=y_{n+2}=0$ as required for $U=A V$ and $A=A\left(A_{2}, A_{n}\right)$ we obtain in addition $x_{n+4+i}=x_{i}, y_{n+4+i}=y_{i}$. Again, $n+4$ is the sum of the Coxeter number of $A_{2}$ and $A_{n}$. The result also applies to the special case $A\left(T_{1}, A_{n}\right)$, since $h\left(T_{1}\right)=h\left(A_{2}\right)$.

So far, we always obtained real values for the solutions of our algebraic equations. Now let us apply the previous equations to the case $A\left(A_{2}, A_{3}\right)$. We write all $x_{i}, y_{i}$ as polynomials in $x, y$ and have to impose the algebraic conditions $x_{4}=y_{4}=1$. This yields $x_{3} x_{5}=y_{3} y_{5}=0$, thus $x_{5}=y_{5}=0$ by admissability. Let us first remark that explicit solvability in terms of roots of unity can only be expected in the admissible case. When $y=0$, but no other component vanishes, we obtain $x^{5}+x^{3}+2 x^{2}-x+1=0$, an equation which does not seem to have magical properties.

We can assume that $x \neq y$ since otherwise we are in the $A\left(T_{1}, A_{3}\right)$ case which has been treated above. The polynomials $\left(x_{4}-y_{4}\right) /(x-y), x_{4}+y_{4}-2$, $\left(x_{5}-y_{5}\right) /(x-y)$ are symmetric under interchange of $x, y$ and can be expressed in the variables $x y$ and $z=x+y$. This yields $z^{3}-z+2-x y(2 z+3)=0$, $(x y)^{2}-x y\left(z^{2}+1\right)+z^{2}-1=0,(x y)^{2}-x y\left(3 z^{2}+4 z+3\right)+z^{4}+3 z+2=$ 0 . By elimination of $x y$ it is easy to see that the only common solution is $z=-1, x y=2$. This yields $(2 x, 2 y)=(-1+i \sqrt{7},-1-i \sqrt{7})$, up to complex conjugation.

Logarithms can be taken such that the relation $U=A V$ is satisfied. For example one may take

$$
\begin{aligned}
& \log \left(x_{1}\right)=\frac{\log (2)-(\pi+\alpha) i}{2} \\
& \log \left(x_{2}\right)=-\pi i \\
& \log \left(x_{3}\right)=\frac{\log (2)+(\pi+\alpha) i}{2}-2 \pi i
\end{aligned}
$$


and $\log \left(y_{i}\right)=\log \left(x_{4-i}\right)$. Applying Roger's dilogarithm to the resulting torsion element $\sum_{i}\left(u_{i}, v_{i}\right)$ of the Bloch group one finds

$$
2 \Re L\left(\frac{3+i \sqrt{7}}{8}\right)=\pi\left(\arg \left(\frac{3+i \sqrt{7}}{8}\right)+\arg \left(\frac{5+i \sqrt{7}}{8}\right)\right)+\pi^{2} / 4 .
$$

The phases arise, because it is not possible to choose $\log (x)$ complex conjugate to $\log (y)$.

As last example before the the introduction of some Lie algebra theory let us consider the case $A=A\left(A_{3}, A_{\infty}\right)$. For aesthetic reasons we label the variables as $z_{1 i}=x_{i}, z_{2 i}=y_{i}, z_{3 i}=z_{i}$ without worrying about the previous use of $z_{i}$. The equations are

$$
\begin{aligned}
& x_{i}^{2}=y_{i}+x_{i-1} x_{i+1} \\
& y_{i}^{2}=x_{i} z_{i}+y_{i-1} y_{i+1} \\
& z_{i}^{2}=y_{i}+z_{i-1} z_{i+1}
\end{aligned}
$$

with $x_{0}=y_{0}=z_{0}=1$. We put $x=x_{1}, y=y_{1}, z=z_{1}$. We will see that in the admissible case

$$
x_{i+1}=x x_{i}-y x_{i-1}+z x_{i-2}-x_{i-3}
$$

for $i=1,2, \ldots$, with $x_{i}=0$ for $i<0$. Of course $x, z$ can be interchanged by the symmetry of $A_{3}$. The recursion for $y_{i}$ is more complicated. One obtains

$$
\begin{aligned}
y_{i+1}= & y y_{i}+x z y_{i-1}-y y_{i-2}+y_{i-3} \\
& +\left(x^{2}+z^{2}\right)\left(y_{i-2}+y_{i-4}+y_{i-6}+\ldots\right) \\
& -2 x z\left(y_{i-1}+y_{i-3}+y_{i-5}+\ldots\right)
\end{aligned}
$$

We show by induction that these additive recursion formulas imply the multiplicative ones, assuming the latter for $j \leq i$. The proof is somewhat tedious to follow. As before, one has to substitute the additive recursion formulas for the $x_{i}$ at many places of the equations. To indicate where the substitutions happen we write $\xi=x$ at the relevant positions. One has

$$
\begin{aligned}
x_{i+1}^{2}-x_{i} x_{i+2}= & x_{i+1}\left(x x_{i}-y x_{i-1}+z x_{i-2}-x_{i-3}\right) \\
& -x_{i}\left(x x_{i+1}-y x_{i}+z x_{i-1}-x_{i-2}\right) \\
= & y\left(x_{i}^{2}-x_{i-1} x_{i+1}\right)+z\left(\xi_{i+1} x_{i-2}-\xi_{i} x_{i-1}\right) \\
& -\xi_{i+1} x_{i-3}+\xi_{i} x_{i-2} \\
= & y y_{i}-x z y_{i-1}+z^{2} y_{i-2}+z\left(x_{i-4} x_{i-1}-x_{i-3} x_{i-2}\right) \\
& -x\left(x_{i} x_{i-3}-x_{i-1} x_{i-2}\right)-y y_{i-2}+y_{i-3} .
\end{aligned}
$$

To prove that the last expression is equal to $y_{i+1}$ one has to show 


$$
\begin{aligned}
& z\left(x_{i-1} x_{i-4}-x_{i-2} x_{i-3}\right)-x\left(x_{i} x_{i-3}-x_{i-1} x_{i-2}\right)= \\
& \quad x^{2}\left(y_{i-2}+y_{i-4}+y_{i-6}+\ldots\right)-2 x z\left(y_{i-3}+y_{i-5}+\ldots\right)+z^{2}\left(y_{i-4}+y_{i-6}+\ldots\right)
\end{aligned}
$$

The brackets on the left hand side have identical structures, except for a shift of the indices by 1 . Thus it is sufficient to calculate one of them. One finds

$$
\begin{aligned}
\xi_{i} x_{i-3}-\xi_{i-1} x_{i-2} & =-x y_{i-2}+z y_{i-3}+\left(\xi_{i-2} x_{i-5}-\xi_{i-3} x_{i-4}\right) \\
& =-x\left(y_{i-2}+y_{i-4}+\ldots\right)+z\left(y_{i-3}+y_{i-5}+\ldots\right)
\end{aligned}
$$

where an obvious induction has been used in the last step. This finishes the proof of the formula for $x_{i+1}^{2}$. Interchange of $x, z$ yields the one for $z_{i+1}^{2}$. For $y_{i+1}^{2}$ a somewhat different approach is easier. We use the equality $x_{i}^{2}=$ $y_{i}+x_{i-1} x_{i+1}$ to express $y_{i}$ in terms of the $x_{j}$. This yields

$$
y_{i}^{2}-y_{i-1} y_{i+1}=x_{i} P_{i}
$$

where

$$
P_{i}=x_{i}^{3}-2 x_{i-1} x_{i} x_{i+1}+x_{i-1}^{2} x_{i+2}+x_{i-2} x_{i+1}^{2}-x_{i-2} x_{i} x_{i+2} .
$$

Thus it suffices to show $P_{i}=z_{i}$ by induction. Now

$$
\begin{aligned}
P_{i+1}= & \xi_{i+3}\left(x_{i}^{2}-x_{i-1} x_{i+1}\right)+\xi_{i+2}\left(x_{i-1} x_{i+2}-x_{i} x_{i+1}\right) \\
& +\xi_{i+1}\left(x_{i+1}^{2}-x_{i} x_{i+2}\right) \\
= & \left.z P_{i}+\xi_{i+2}\left(x_{i} x_{i-3}-x_{i-1} x_{i-2}\right)+\xi_{i+1}\left(x_{i-1}\right)^{2}-x_{i+1} x_{i-3}\right) \\
& +\xi_{i}\left(x_{i-2} x_{i+1}-x_{i} x_{i+2}\right) \\
= & z z_{i}-y P_{i-1}+\xi_{i+1}\left(x_{i-3}^{2}-x_{i-2} x_{i-4}\right)+\xi_{i}\left(x_{i-1} x_{i-4}-x_{i-2} x_{i-3}\right) \\
& +\xi_{i-1}\left(x_{i-2}^{2}-x_{i-1} x_{i-3}\right) \\
= & z z_{i}-y z_{i-1}+x z_{i-2}-z_{i-3}=z_{i+1}
\end{aligned}
$$

which finishes the proof.

Applying the linear recursion relation to negative values we obtain $x_{-i}=$ $-z_{i-4}$ and $y_{-i}=-y_{i-4}$. For solutions of $U=A V$ with $A=A\left(A_{3}, A_{n}\right)$ we also find the periodicity $x_{i+n+5}=x_{i}$ and analogously for $y, z$.

At this stage the reader should be convinced that elementary algebra is not entirely satisfactory for an understanding of what is going on. Obviously, the generalisation of the linear recursion for $x_{i}$ to $A\left(A_{m}, A_{\infty}\right)$ is easy to guess,. and maybe even to prove. When one looks at the coefficients, one gets a path through the Dynkin diagram of $A_{m}$ which starts at one side and ends at the other. This is one of Ocneanu's essential paths O99, namely the one which starts at the $x$-vertes. For the $y_{i}$ terms in $A\left(A_{3}, A_{\infty}\right)$ we first go from $y$ to $x, z$ and then back to $y$. Again this agrees with Ocneanu's essential path starting at the $y$-vertex. Maybe this generalizes, but it doesn't give us the chain of correction terms, which certainly will become more complicated for Lie algebras of higher rank. 
Thus we will leave this approach and jump to conclusions which for us come a bit out of the blue, but do work K87, KR87. We need another interpretation of the algebraic equations which gives a new meaning to the solutions. Since the latter are sums of roots of unity with integral coefficients, one can expect that these integers count something. For the Chebysheff polynomials we have $p_{i}(2)=i+1$, numbers which will be interpreted as the dimensions of the irreducible representations of $A_{1}$. Dimensions can be obtained as character values at the unit element, and more generally the algebraic integers will be given as character values at elements of finite order. Apart from some general remarks, we shall only consider the case $A\left(A_{m}, A_{n}\right)$, where it suffices to work with Lie groups instead of quantum groups. In this case the argumentation will be quite self-contained, apart from the fact that the reader is supposed to know or look up the Littlewood-Richardson rules. On the other hand, we briefly recall most of the relevant facts of representation theory. The Weyl character formula is mentioned to provide some context, but its relevant special cases are discussed without reference to the general theory. We will work over the complex numbers and in the context of algebraic groups, which avoids many unnecessary complications.

Let us first consider the irreducible finite dimensional representations of $G L(n)$. In particular, the subgroup of diagonal matrices, with elements $g=$ $\operatorname{diag}\left(a_{1}, \ldots, a_{n}\right)$ will be represented. Its irreducible representations are onedimensional and map $g$ to $\prod_{k=1}^{n} a_{k}^{i_{k}}$, with integral $i_{k}$. Such a representation is called a weight and notated as a sequence $I=\left(i_{1}, \ldots, i_{n}\right)$. One-dimensional representations can be multiplied and inverted. In terms of the sequences $I$ this group structure is additive - the weights form a lattice. When restricted to the diagonal elements, the representations $\rho$ of $G L(n)$ decompose into a finite number of weights. The diagonal matrices $g=\operatorname{diag}(a, \ldots, a)$ form the center of $G L(n)$. They are represented by $a^{|I|}$, where $|I|=i_{1}+\ldots+i_{n}$. When $\rho$ is irreducible, the center is represented by multiples of the identity matrix, such that $|I|$ takes the same value for all weights occuring in $\rho$.

Let the $G L(n)$ representations $\rho, \rho^{\prime}$ of dimensions $d, d^{\prime}$ decompose into weights $I_{r}, r=1, \ldots, d$ and $I_{s}^{\prime}, s=1, \ldots, d^{\prime}$. Then the tensor product $\rho \otimes$ $\rho^{\prime}$ decomposes into weights $I_{r}+I_{s}^{\prime}$. The symmetric power $S^{k} \rho$ decomposes into weights $I_{r_{1}}+\ldots+I_{r_{k}}$ with $r_{1} \geq \ldots \geq r_{k}$ and the external power $\Lambda^{k} \rho$ decomposes into weights $I_{r_{1}}+\ldots+I_{r_{k}}$ with $r_{1}>\ldots>r_{k}$.

The character $\chi: G L(n) \rightarrow \mathbb{C}$ of a representation $\rho$ of dimension $d$ is given by $\chi(g)=\operatorname{Tr} \rho(g)$. It has the form

$$
\chi(g)=\sum_{I} \prod_{k=1}^{n} a_{k}^{i_{k}},
$$

where the sum goes over the $d$ weights occuring in $\rho$. With a sequence $I=$ $\left(i_{1}, \ldots, i_{n}\right)$ all its permutations appear, too, since permutations of the entries of $g$ can be achieved by conjugation in $G L(n)$. When one gives the standard lexicographic order to the $\left(i_{1}, \ldots, i_{n}\right)$, any irreducible representation of $G L(n)$ 
has a unique highest weight. Since any sequences $\left(i_{1}, \ldots, i_{n}\right)$ can be permuted to a decreasing one, such a highest weight is given by a decreasing sequence of $n$ integers. One can show that all such sequences can occur as highest weights. This yields a complete classification of the isomorphism classes of irreducible $G L(n)$ representations. We write the corresponding representation as $\rho_{I}$, its character as $\chi_{I}$.

The character of the defining representation of $G L(n)$ maps $g$ to $\operatorname{Tr}(g)=$ $\sum_{i=1}^{n} a_{n}$. Thus its weights are $(1,0, \ldots, 0)$ and its permutations, and its highest weight is $(1,0, \ldots, 0)$. The $k$-th symmetric product of this representation is irreducible and has highest weight $(k, 0, \ldots, 0)$. Its $k$-th exterior product is irreducible and has highest weight $(\underbrace{1, \ldots, 1}_{k \text { terms }}, 0, \ldots, 0)$. For $k>n$ the exterior product is 0 , for $k=n$ it yields the one-dimensional determinant representation, with unique weight $(1, \ldots, 1)$. For $I=(\underbrace{i, \ldots, i}_{k \text { terms }}, 0, \ldots, 0)$ we write $\rho_{I}=\rho_{i}^{k}$ and $\chi_{I}=\chi_{i}^{k}$

The character of the irreducible $G L(n)$ representation with highest weight $I=\left(i_{1}, \ldots, i_{n}\right)$ is given by

$$
\chi_{I}(g)=D_{I}(g) / D_{0}(g),
$$

where $D_{I}(g)=\operatorname{det}\left(M^{I}\right)$ and the matrix $M^{I}$ has entries

$$
M_{l r}^{I}=a_{r}^{i_{l}+n-l}
$$

$l, r=1, \ldots, n$. This is a special case of the Weyl character formula. For $I=$ $(\underbrace{i, \ldots, i}_{k \text { terms }}, 0, \ldots, 0)$ we often write $\rho_{I}=\rho_{i}^{k}$ and analogously for $\chi_{I}$ and $M^{I}$.

Since $\rho_{i}^{1}$ is the $i$-th symmetric product of $\rho_{1}^{1}$, one has

$$
\chi_{i}^{1}(g)=\sum_{\substack{m_{1}, \ldots, m_{n} \in \mathbb{N} \\ m_{1}+\ldots m_{n}=i}} \prod_{r=1}^{n} a_{r}^{m_{r}}
$$

To check the Weyl character formula in this case, one first notes that $D_{0}$ is a Vandermonde determinant, such that

$$
D_{0}(g)=\prod_{r<s}\left(a_{r}-a_{s}\right)
$$

In our case the matrix $M^{I}$ agrees with $M^{0}$ apart from the first row, which has entries $a_{r}^{i+n-1}$. Its determinant can de developed in terms of minors with respect to the first row. Up to a sign, the minor multiplying $a_{r}^{i+n-1}$ is $D_{0}\left(g_{r}\right)$, where the $g_{r}$ are diagonal matrices in $G L(n-1)$ and arise from $g$ by supressing $a_{r}$. One can that this development agrees with $D_{0}(g) \chi_{i}^{1}(g)$. Indeed

$$
\left(a_{1}-a_{2}\right) \chi_{i}^{1}(g)=\chi_{i+1}^{1}\left(g_{2}\right)-\chi_{i+1}^{1}\left(g_{1}\right) .
$$


By induction one sees immediately that

$$
\prod_{r=2}^{s}\left(a_{1}-a_{r}\right) \chi_{i}^{1}(g)=\chi_{i+s-1}^{1}\left(g^{s}\right)+R_{s}
$$

where the $g^{s}$ are diagonal matrices in $G L(n-s+1)$ which arise from $g$ by supressing $a_{2}, \ldots, a_{s}$ and the $a_{1}$ degree of $R_{s}$ is at most $s-2$. Using this result for $s=n$, where $\chi^{i+n-1}\left(a_{1}\right)=a_{1}^{i+n-1}$, one sees that $D_{0}(g) \chi_{i}^{1}(g)-D_{i}^{1}(g)$ has degree at most $n-2$ in $a_{1}$ and by permutation symmetry in all $a_{r}$. When one looks at the developments in first row minors of $D_{i}^{1}(g)$ and $D_{0}(g)$, one sees that such terms cannot occur, such that $D_{0}(g) \chi_{i}^{1}(g)-D_{i}^{1}(g)=0$.

When one restricts $G L(n)$ to $S L(n)$, the determinant representation becomes trivial. Thus weights $\left(i_{1}, \ldots, i_{n}\right)$ and $\left(i_{1}+j, \ldots, i_{n}+j\right), j \in \mathbb{Z}$, become equivalent, and one can regard the sequences $\lambda=\left(i_{1}-i_{2}, \ldots, i_{n-1}-i_{n}\right)$ as the weights of $S L(n)$. All such integral can occur, since each irreducible representation of $S L(n)$ can be lifted to $G L(n)$. For the highest weights of $S L(n)$ representations, all entries are non-negative. These highest weights form a semi-group which is generated by the $n-1$ fundamental weights $(1,0, \ldots, 0),(0,1,0, \ldots, 0), \ldots(, 0, \ldots, 1)$. The corresponding representations are just the exterior products of the defining representation mentioned above, with $k=1, \ldots, n-1$. These are the fundamental representations of $S L(n)$. The Dynkin diagram of $S L(n)$ is an ordered chain of $n-1$ vertices, which can be labelled by $k=1, \ldots, n-1$. Thus each of them corresponds to one of the fundamental representations, and each irreducible representation of $S L(n)$ can be classified up to isomorphism by associating natural numbers $i_{k}-i_{k+1}$ to the corresponding vertices.

The Dynkin diagram $A_{n-1}$ of $S L(n)$ has an obvious reflection symmetry. In terms of the characters this yields in particular

$$
\chi_{i}^{k}\left(g^{-1}\right)=\chi_{i}^{n-k}(g) .
$$

Instead of the Lie groups $G L(n)$ and $S L(n)$ one can consider their Lie algebras. In both cases, the Lie algebra of the subgroup of diagonal matrices forms a maximal abelian subalgebra. The classification of the irreducible representations of the Lie algebras is the same as for the Lie groups.

The procedure generalizes to the other simple Lie algebras $X$ and their finite dimensional representations. As for $G L(n)$ and $S L(n)$ one chooses a Cartan subalgebra, i.e. a maximal abelian subalgebra of $X$. Its irreducible representations are called weights and form a lattice $\Lambda$ isomorphic to $\mathbb{Z}^{r}$, where $r$ is the rank of $X$. The vertices of the Dynkin diagram of $X$ correspond to a basis of the dual lattice of $\Lambda$, such that weights yield an integer for each vertex. The Cartan matrix corresponding to the Dynkin diagram yields a metric on the dual of $\Lambda$ and therefore on $\Lambda$ itself.

The weight lattice can be ordered in some lexicographic way. The irreducible representations of $X$ have highest weights, which give a natural number (possibly zero) for each vertex of the Dynkin diagram. This yields a classification of the isomorphism classes of these representations. In particular, 
they form a semi-group. Choosing zero for each vertex yields the trivial onedimensional representation, the zero of this semigroup. Choosing one for some vertex $k$ and zero for the others yields the fundamental representations with highest weights $\lambda^{k}$.

The tensor product $\rho \otimes \rho^{\prime}$ of two irreducible representations $\rho, \rho^{\prime}$ decomposes into a direct sum of irreducible representations. Among their weights one is the highest, and given by the sum of the highest weights of $\rho$ and $\rho^{\prime}$. For a representation $\rho$ the corresponding character $\chi: X \rightarrow \mathbb{C}$ is given by $\chi(x)=\operatorname{Tr} \rho(x)$. We also use $\chi$ for the Lie group representations obtained by exponentiating the $\rho(x)$. The character of $\rho \otimes \rho^{\prime}$ is the ordinary product $\chi \chi^{\prime}$. The dimension of $\rho$ is equal to $\chi(1)$.

After this extensive recall of Lie algebra theory we can come back to our algebraic equations. For $X=A_{1}$ the Dynkin diagram has one vertex only, so the irreducible representations $\rho_{i}$ are classified by a single natural number $i=0,1,2, \ldots$. The dimension of $\rho_{i}$ turns out to be $i+1$. We have $\chi_{i}=$ $p_{i}\left(\chi_{1}\right)$, where the $p_{i}$ are the Chebysheff polynomials. A check can be made by evaluating this relation at $g=1$, where one indeed has $i+1=p_{i}(2)$, which is the correct dimension. To prove the formula one can use the well-known tensor product relation

$$
\rho_{1} \otimes \rho_{i}=\rho_{i-1} \oplus \rho_{i+1}
$$

for $i>0$, which yields

$$
\chi_{1} \chi_{i}=\chi_{i-1}+\chi_{i+1}
$$

This agrees with the recursion relation for the $p_{i}(x)$. Since also $p_{0}\left(\chi_{1}\right)=$ $\chi_{0}=1$ and $p_{1}\left(\chi_{1}\right)=\chi_{1}$, it is clear that $\chi_{i}=p_{i}\left(\chi_{1}\right)$. The multiplicative recursion relation for the $p_{i}$ can be explained in the same way, since $\rho_{i} \otimes \rho_{i}=$ $\rho_{0} \oplus \rho_{2} \oplus \cdots \oplus \rho_{2 i}$ and $\rho_{i-1} \otimes \rho_{i+1}=\rho_{2} \oplus \cdots \oplus \rho_{2 i}$.

For $X=A_{m}$, let us consider the $G L(m+1)$ representations $\rho_{i}^{k}$ introduced above. Like all irreducible representation of $G L(m+1)$, they stay irreducible when the representation is restricted to $S L(m+1)$ or its Lie algebra $A_{m}$. Indeed, the elements of $G L(m+1)$ are products of $S L(m+1)$ elements and a elements in the center, and in irreducible representations the latter are represented by multiples of the identity operator. The $\chi_{i}^{k}$ can be written as polynomials in the $\chi_{1}^{k}$. It turns out that these polynomials coincide with the ones we obtained for $A\left(A_{m}, A_{\infty}\right)$. Moreover we can write $z_{k i}=\chi_{i}^{k}(g)$, where $g \in S L(m+1)$ is determined up to conjugation by $z_{k 1}=\chi_{1}^{k}(g)$.

To prove this result we must to show that for $i=1, \ldots, m$ and $k=1,2, \ldots$ one has

$$
\rho_{i}^{k} \otimes \rho_{i}^{k}=\left(\rho_{i+1}^{k} \otimes \rho_{i-1}^{k}\right) \oplus\left(\rho_{i}^{k+1} \otimes \rho_{i}^{k-1}\right) .
$$

Here the $\rho_{0}^{k}$ and the $\rho_{i}^{0}$ are triviall one-dimensional representations. The $\rho_{i}^{m+1}$ are the $i$-th powers of the determinant representation, which becomes trivial for $g \in S L(m+1)$.

The formula follows immediately from the Littlewood-Richardson rules for tensor products of irreducible representations of $G L(m+1)$, but here only a 
brief sketch of the derivation will be given. As we have seen, these representations are classified by decreasing sequences $I=\left(j_{1}, \ldots, j_{m+1}\right)$ of integers. The one-dimensional determinant representation is given by $(1, \ldots, 1)$ and its tensor product with the representation of highest weight $\left(j_{1}, \ldots, j_{m+1}\right)$ yields the highest weith $\left(j_{1}+1, \ldots, j_{m+1}+1\right)$.

We are only interested in representations with $j_{m+1} \geq 0$. For notational convenience we represent the corresponding highest weights as infinite sequences $I=\left(j_{1}, \ldots, j_{m+1}, 0,0, \ldots\right)$. We define the length $l(I)$ of $I$ as the number of its non-zero terms and we let all sequences with length $l(I)>m+1$ correspond to the 0 representation. Then the $\rho_{i}^{k}(\underbrace{i, \ldots, i}_{k \text { times }}, 0, \ldots)$. The Littlewood-

Richardson rules imply that $\rho_{i}^{k} \otimes \rho_{i}^{k}$ is the direct sum of the representations given by $\left(i+j_{1}, \ldots, i+j_{k}, i-j_{k}, \ldots, i-j_{1}, 0, \ldots\right)$, where $\left(i, j_{1}, \ldots, j_{k}, 0\right)$ is a decreasing integral sequence. Similarly $\rho_{i+1}^{k} \otimes \rho_{i-1}^{i}$ corresponds to the direct sum of the subset of these representations for which $j_{k}=0$ and $\left(\rho_{i}^{k+1} \otimes \rho_{i}^{k-1}\right.$ corresponds to the complementary subset. This proves our equality.

The linear recursion relations for the $\rho_{i}^{1}$ are easy to prove, too. According to the Littlewood-Richardson relations the tensor product of $\rho_{i}^{1}$ with $\rho_{1}^{k}$ is given by the sum of two irreducible representations with highest weights $(i+$ $1, \underbrace{1, \ldots, 1}_{k-1 \text { times }})$ and $(i, \underbrace{1, \ldots, 1}_{k \text { times }})$. This immediately yields

$$
\begin{array}{r}
\left(\rho_{i}^{1} \otimes \rho_{1}^{1}\right) \oplus\left(\rho_{i-2}^{1} \otimes \rho_{1}^{3}\right) \oplus\left(\rho_{i-4}^{1} \otimes \rho_{1}^{5}\right) \oplus \ldots= \\
\rho_{i+1}^{1} \oplus\left(\rho_{i-1}^{1} \otimes \rho_{1}^{2}\right) \oplus\left(\rho_{i-3}^{1} \otimes \rho_{1}^{4}\right) \oplus \ldots .
\end{array}
$$

which in turn yields for $i \geq m$.

$$
\chi_{i+1}^{1}=\chi_{1}^{1} \chi_{i}^{1}-\chi_{1}^{2} \chi_{i-1}^{1}+\ldots+(-)^{m} \chi_{1}^{m+1} \chi_{i-m}^{1} .
$$

This formula can be interpreted as a recursion relation for the $\chi_{i}^{1}$. It stays true for $i=0, \ldots, m-1$, if one puts $\rho_{i}^{1}=0$ for $i=-1,-2, \ldots,-m$. This result agrees with what one obtains from a continuation of the Weyl character formula for $\chi_{i}^{1}$ to negative values of $i$. Indeed the matrices $M_{i}^{1}$ have two equal rows for $i=-1,-2, \ldots,-m$, such that their determinants vanish.

We have seen that for $g \in S L(m+1)$ the multiplicative recursion relations for the $\chi_{i}^{k}(g)$ agree with those of the $z_{k i}$. For admissible solutions of the algebraic equations, the $z_{k i}$ are uniquely determined by the $z_{k 1}$. Thus it remains to show that every choice of the $z_{k 1}$ can be parametrised in the form $z_{k 1}=\chi_{1}^{k}(g)$. Since the $\chi_{1}^{k}(g)$ are just the elementary symmetric polynomials of the $a_{1}, \ldots, a_{m+1}$ this is indeed possible. When one leaves $z_{m+1,1}$ free one has a bijection between the $z_{k 1}$ and the $a_{1}, \ldots, a_{m+1}$, the latter taken over $\mathbb{C}$ and up to permutations. The condition $z_{m+1,1}=1$ restricts $\operatorname{diag}\left(a_{1}, \ldots, a_{m+1}\right)$ to $S L(m+1)$.

To find admissible solutions of our algebraic equations for $A\left(A_{m}, A_{n}\right)$ we need $\chi_{n+1}^{k}(g)=1, \chi_{n+2}^{k}(g)=0$ for $k=1, \ldots, m$. We first will show that this implies $\chi_{n+1+l}^{k}(g)=0$ for $l=1, \ldots, m$. 
More generally, for such $l$ we will show $\chi_{I}(g)=0$, if $j_{1}>n+1, j_{1}+l(I)=$ $n+l+2,|I| \geq l+l(I)(n+1)$. Here $\chi_{I}$ is the character of the representation with highest weight $I=\left(j_{1}, j_{2}, \ldots\right)$. Recall that $|I|$ is the sum of the $j_{k}$. Note that the inequalities imply $l(I) \leq m$.

The proof works by induction in $l, j_{1}$, in lexicographic order. For the smallest allowed value $j_{1}=n+2$ we have $l(I)=l$. This yields $|I| \leq l(n+2)$, since $I$ is a decreasing sequence. On the other hand $|I| \geq l+l(I)(n+1)=l(I)(n+2)$. Thus the inequalities are saturated and

$$
I=(\underbrace{n+2, \ldots, n+2}_{l \text { terms }}, 0, \ldots) .
$$

Thus $\chi_{I}=\chi_{n+2}^{l}$ and we have nothing to prove.

In general, let $I$ be strictly decreasing after exactly $r$ terms, such that $j_{r}=j_{1}, j_{r+1}<j_{1}$. For $r=l$ we have $l(I)=l$ and $j_{1}=n+2$, so we are in the previous case. Thus we may assume $r<l$. By assumption, this implies $j_{1}>n+2$.

Now the Littlewood-Richardson rules yield

$$
\begin{aligned}
\rho_{I} \otimes & \rho_{n+1}^{1} \\
& =\left(\rho_{I-\alpha_{1}} \otimes \rho_{n+2}^{1}\right) \oplus-\left(\rho_{I-\alpha_{2}} \otimes \rho_{n+3}^{1}\right) \oplus \ldots \oplus(-)^{r+1}\left(\rho_{I-\alpha_{r}} \otimes \rho_{n+1+r}^{1}\right) \\
& \bigoplus_{I^{\prime}}( \pm) \rho_{I^{\prime}}
\end{aligned}
$$

where minus signs are interpreted in the form $\rho-\rho=0$, the $\alpha_{k}$ have the form

$$
\alpha_{k}=(\underbrace{0, \ldots, 0}_{r-k \text { terms }}, \underbrace{1, \ldots, 1}_{k \text { terms }}, 0, \ldots)
$$

and the highest weights $I^{\prime}$ satisfy $j_{1}^{\prime}=j_{1}-1, l\left(I^{\prime}\right) \leq l(I)+1,\left|I^{\prime}\right|=|I|+n+1$. In particular $j_{1}^{\prime}>n+1, j_{1}^{\prime}+l\left(I^{\prime}\right) \leq n+l+2,\left|I^{\prime}\right| \geq l+l\left(I^{\prime}\right)(n+1)$, such that we can use the induction hypothesis $\chi_{I^{\prime}}(g)=0$. By the induction hypothesis we also have $\chi_{n+k+1}^{1}(g)=0$ for $k=1, \ldots, r$. Together with $\chi_{n+1}^{1}(g)=1$ this yields $\chi_{I}(g)=0$, and the proof is finished.

Next we will show that the algebraic equations imply that $D_{0}(g) \neq 0$, such that the eigenvalues $a_{r}$ of $g$ must all be different. We assume $n \geq m$, otherwise we exchange $A_{m}$ and $A_{n}$. We have

$$
a_{n+1} \chi_{n+2}^{1}(g)-\chi_{n+3}^{1}(g)=\chi_{n+3}^{1}\left(g_{n+1}\right),
$$

where $\left(g_{n+1} \in G L(n)\right.$ is given by $g=\operatorname{diag}\left(a_{1}, \ldots, a_{n}\right)$. By iteration we find that $\chi_{n+m+1}^{1}\left(\left(a_{1}, a_{2}\right)\right)$ is a linear combination of $\chi_{n+1+l}(g), l=1, \ldots, m$ and thus has to vanish. On the other hand

$$
\chi_{n+m+1}^{1}\left(\left(a_{1}, a_{2}\right)\right)=a_{1}^{n+m+1}+a_{1}^{n+m} a_{2}+\ldots+a_{2}^{n+m+1}
$$


and the right hand side cannot vanish for $a_{1}=a_{2}$, unless $a_{1}=0$, which is not permitted by $g \in G L(n)$. By permutation symmetry, all $a_{r}$ have to be different.

Use of the linear recursion relation for the $\chi_{i}^{1}$ allows to express $\chi_{n+m+2}^{1}$ in terms of $\chi_{n+1+l}^{1}$ with $l=0, \ldots, m$. This yields $\chi_{n+m+2}^{1}(g)=(-)^{m}$ for the solutions of our algebraic equations, thus $D_{n+m+2}^{1}(g)=(-)^{m} D_{0}^{1}(g)$. More generally,

$$
D_{i+n+m+2}^{1}(g)=(-)^{m} D_{i}^{1}(g)
$$

for $i=-m, \ldots, 0$, since both sides vanish for $i=-m, \ldots, 1$. Rows 2 to $m+1$ of the $M_{i}^{1}$ are given by the same Vandermonde matrix. The first row has entries $a_{k}^{m+i}$. Developing the determinants $D_{i}^{1}$ into minors with respect to the first row yields a system of $m+1$ linear equations for the $a_{k}^{m+n+2}, k=1, \ldots, m+1$. The determinant of this system is a product of Vandermonde matrices, thus non-zero. This yields the unique solution $a_{k}^{m+n+2}=1$ for all $k$.

These conditions are necessary for admissible solutions of our algebraic equations. To show that they actually solve the equations we just have to notice that they imply

$$
D_{i+n+m+2}^{k}(g)=(-)^{m} D_{i}^{k}(g)
$$

for all $k$. For $i=-m-1$ and $D_{0}(g) \neq 0$ this yields $\chi_{n+1}^{k}(g)=1$, such that the equations are satisfied. Admissibility has to be investigated separately, but this will not be done here.

We can formulate our result in the following way. Admissible solutions of our algebraic eqations for $A\left(A_{m}, A_{n}\right)$ can be written as $\chi_{i}^{k}(g)$, where $g$ is an element of $S U(m+1)$ which satisfies

$$
g^{m+n+2}=(-)^{m}
$$

and all of whose eigenvalues are different. Note that $m+n+2$ must be the smallest power of $g$ which is equal to 1 or -1 , since for a small power $m+k+2$ with this property one finds $\chi_{i}^{k+2}(g)=0$. The reader is encouraged to recover the solutions for the special cases $A\left(A_{1}, A_{m}\right)$ and $A\left(A_{2}, A_{3}\right)$ discussed above from our general result for $A\left(A_{m}, A_{n}\right)$.

Before we leave this case, we consider two symmetry properties of our solutions. Applying the symmetry of the $A_{m}$ or $A_{n}$ diagram to any solution yields another solution. We shall see that simultaneous application of these two operations leaves any solution invariant.

By the symmetry of the $A_{m}$ diagram, we have the same linear recursion relation for the $\chi_{i}^{m}$, except that the coefficients $\chi_{1}^{k}$ are replaced by $\chi_{1}^{m+1-k}$. For the solutions of our algebraic equations this means that they obey linear recursion relations which are invariant under simultaneous application of the symmetries of $A_{m}$ and $A_{n}$. In the special cases $A=A\left(A_{m}, A_{n}\right), m=1,2,3$ considered above we have found the same symmetry in all admissible solutions themselves. We will prove that this remains true for all $\mathrm{m}$. The coefficients of 
the linear recursion relation for the $\chi_{i}^{1}(g)$ are the $\chi_{1}^{k}(g), k=1, \ldots m$. When one reads the relations in the opposite sense, as relations among the $\chi_{-i}^{1}(g)$, the coefficients become $\chi_{1}^{m+1-k}(g)=\chi_{1}^{k}\left(g^{-1}\right)$. Use of the $A_{m}$ symmetry yields linear recursion relations for the $\chi_{-i}^{m}(g)$, but now with the original coefficients $\chi_{1}^{k}(g)$.

The linear recursion relations allow us to express $\chi_{i+m+1}^{1}(g)$ in terms of $\chi_{i+l}^{1}(g)$ with $l=0, \ldots, m$ and analogously $\chi_{i-m-1}^{m}(g)$ in terms of $\chi_{i-l}^{m}(g)$, with the same coefficients. Now for admissible solutions of our algebraic equations we have found $\chi_{i}^{1}(g)=\chi_{n+1-i}^{m}(g)$ for $i=-m, \ldots, 0$. By induction, the linear recursion relations yield the same equality for all values of $i$, in particular the values $i=1, \ldots, m$ we need for the solutions of our algebraic equations. Moreover, the $\chi_{i}^{1}(g)$ or $\chi_{i}^{m}(g)$ determined all $\chi_{i}^{k}(g)$, such that

$$
z_{k i}=z_{m+1-k, n+1-i}
$$

for all $k, i$.

Finally one expects a duality between $A\left(A_{n}, A_{m}\right)$ and $A\left(A_{m}, A_{n}\right)$. Indeed consider $g=\operatorname{diag}\left(a_{1}, \ldots, a_{m+1}\right)$ with $g^{m+n+2}=(-)^{m}$ and no coinciding eigenvalues. The $a_{i}$ form $n+1$ of the $(m+n+2)$-th roots of $(-)^{m}$. Let $\left(a_{m+2}, \ldots, a_{m+n+2}\right)$ be the remaining roots. Then $h=\operatorname{diag}\left(a_{m+2}, \ldots, a_{m+n+2}\right)$ satisfies $h \in S L(n+1)$ and $h^{m+n+2}=(-)^{m}$, as one can check easily. In this way one can lift the restriction $m \leq n$ we used to conclude that $g$ had no degenerate eigenvalues.

The calculations we made for $A\left(A_{m}, A_{n}\right)$ can be extended to $A\left(D_{m}, A_{n}\right)$ and $A\left(E_{m}, A_{n}\right)$, but one has to go beyond Lie algebras into the domain of quantum groups, as shown in K87 KR87. Quantum groups have a co-algebra structure, which allows to define tensor products of representations as in the Lie algebra case. For each simple Lie algebra $X_{m}$ there is a quantum group $Y\left(X_{m}\right)$, called the Yangian of $X_{m}$, which contains the enveloping algebra $U\left(X_{m}\right)$ of $X_{m}$. Recall that the representations of $U\left(X_{m}\right)$ are given in a natural way by the representations of $X_{m}$ itself.

For the special case $X_{m}=A_{m}$ there is a map from $A_{m}$ to $Y\left(A_{m}\right)$ which reduces the representation theory of $Y\left(A_{m}\right)$ to the one of $A_{m}$, since every representation of $A_{m}$ can be extended to a representation of $Y\left(A_{m}\right)$ on the same vector space, and all irreducible representation of $Y\left(A_{m}\right)$ remain irreducible as representations of $U\left(A_{m}\right)$. For $D_{m}$ and $E_{m}$ this no longer true. Nevertheless the representations of $Y\left(X_{m}\right)$ can still be decomposed into weights of $X_{m}$ and irreducible representations can be labelled by their unique highest weight. Tensor products correspond to the addition of weights as for $X_{m}$ itself. Let $\rho_{i}^{k}$ be the representation of $Y\left(X_{m}\right)$ with highest weight $i \lambda^{k}$, where $k$ runs over the vertices of the Dynkin diagram of $X_{m}$. As before, the $z_{k i}=\chi_{k}^{i}(g)$ can be written as polynomials of the $z_{k 1}$ and satisfy the algebraic equations of $A\left(X_{m}, A_{\infty}\right)$. Imposing $\chi_{n+1}^{i}(g)=1, \chi_{n+2}^{i}(g)=0$ should allow to solve the algebraic equations for $A\left(X_{m}, A_{n}\right)$. Indeed the special real solution relevant for calculating the central charge $c$ is known and given by 


$$
g=\exp \left(-\frac{2 \pi i \rho}{h\left(X_{m}\right)+n+1}\right)
$$

where $\rho$ is the sum of all $\lambda^{k}$. Thus $g_{0}=g^{h(X)+n+1}$ lies in the center of the simply connected compact Lie group corresponding to $X$ and satisfies

$$
\rho_{j}^{k}\left(g_{0}\right)=\exp \left(-2 \pi i j\left(\lambda_{k}, \rho\right),\right.
$$

where we have used the natural scalar product on $\Lambda$. One may expect that all solutions of the algebraic equations arise in this way, but the precise conditions on $g$ seem to be still unknown.

\section{Conclusions}

Of the unsolved problems mentioned in this article many are of a purely mathematical nature and seem to be quite accessible. Much more challenging is the development of a mathematically satisfactory theory of integrable quantum field theories in two dimensions. The physics literature provides a wealth or ideas and results, and perhaps it is time that mathematicians get interested. Those theories which arise from perturbations of rational conformal field theories may be the easiest ones to study.

\section{References}

ABD03. E. Ardonne, P. Bouwknegt, And P. Dawson, K-matrices for 2D conformal field theories, Nucl.Phys. B660 (2003), 473-531

A76. G.E. AndRews, The theory of partitions, in: Encyclopedia of Mathematics and its Applications, Vol. 2, Addison Wesley, 1976

BM98. A. BERKOVICH, B.M. MCCOY, The universal chiral partition function for exclusion statistics, hep-th/9808013

D98. P. DOREY, Exact S-matrices, hep-th/9810026

DT98. P. DOREY, R. TATEO, Excited states in some simple perturbed conformal field theories, Nucl.Phys. B515 (1998), 575-623

FS93. E. Frenkel, A. Szenes, Crystal bases, dilogarithm identities and torsion in algebraic K-groups, hep-th/9304118

FS95. E. Frenkel, A. Szenes, Thermodynamic Bethe ansatz and dilogarithm identities $I$, hep-th/9506215

GZ00. H. GANGL, D. ZAGIER, Classical and elliptic polylogarithms and special values of L-Series, in: B.B. Gordon et al. eds., The Arithmetic and Geometry of Algebraic Cycles, 2000

GT96. F. GLiozzI, R. TATEO, Thermodynamic Bethe ansatz and threefold triangulations, Int.J.Mod.Phys. A11 (1996), 4051-4064

GT95. F. GLIOZZI, R. TATEO, ADE functional dilogarithm identities and integrable models, Phys.Lett. B348 (1995), 84-88 
KM93. R. Kedem, T.R. Klassen, B.M. McCoy, E. Melzer, Fermionic quasiparticle representations for characters of $\frac{G_{1}^{(1)} \times G_{1}^{(1)}}{G_{2}^{(1)}}$, Phys.Lett. B304 (1993), 263-270

K87. A.N. KIRILlOv, Identities for the Rogers dilogarithm function connected with simple Lie algebras, J. Soviet Mathematics 47 (1989), 2450-2459

KR87. A.N. Kirillov, N.Yu. Reshetikhin, Representations of Yangians and multiplicities of occurrence of the ireducible components of the tensor product of representations of simple Lie algebras, J. Soviet Mathematics 52 (1990), 3156-3164

KM90. T. Klassen, E. Melzer, Purely elastic scattering theories and their ultraviolet limits, Nucl.Phys. B338 (1990), 485-528

KN92. A. Kuniba, T. NAKAnishi, Spectra in conformal sield theories from the Rogers dilogarithm, Mod.Phys.Lett. A7 (1992), 3487-3494

KNS93. A. Kuniba, T. NAKAnishi, J. Suzuki, Characters in conformal field theories from thermodynamic Bethe ansatz, Mod.Phys.Lett. A8 (1993), $1649-1660$

M91. M.J. MARTINs Complex excitations in the thermodynamic Bethe ansatz, Phys.Rev.Lett. 67 (1991), 419-421

N93. W. NAHM, Conformal field theory, dilogarithms, and three dimensional manifolds, in: Interface between physics and mathematics, Proceedings, Hangzhou 1993, W. Nahm and J.M. Shen eds., World Scientific

NRT93. W. Nahm, A. Recknagel, And M. Terhoeven, Dilogarithm identities in conformal field theory, Mod.Phys.Lett. A8 (1993), 1835-1848

N03. W.D. Neumann, Extended Bloch group and the Cheeger-Chern-Simons class, math.GT/0307092

NZ85. W.D. NEumann, D. ZAGIER, Volumes of hyperbolic 3-manifolds, Topology 24 (1985), 307-332

O99. A. OCNEAnu, Paths on Coxeter diagrams: From Platonic solids and singularities to minimal models and subfactors, AMS Fields Institue Monographs no. 13, (1999), B.V. Rajarama Bhat, G,A, Elliott, P.A. Fillmore eds., vol. 'Lectures on Operator Theory'

VK90. V. KAC, Infinite dimensional Lie algebras, Third edition, Cambridge University Press, Cambridge 1990

S86. A.A. Susuin, Algebraic K-theory of fields, ICM, Berkeley, I (1986) 222244

S89. C.H. SAH, Homology of classical Lie groups made discrete, III, J. Pure Appl. Algebra 56 (1989) 313-318

W03. S. WEINZIERL, Algebraic algorithms in perturbative calculations, hep-th/0305260

DZ91. D. ZAGIER, Polylogarithms, Dedekind zeta functions and the algebraic Ktheory of fields, in: Progr. Math. 89 (1991), Birkhäuser Boston, Boston MA, 391-430

Z89. A.B. ZAmolodchikov, Integrable field theory from conformal fiel theory, Adv. Stud. in Pure Math. 19 (1989), 641

Z91. A.B. Zamolodchikov, On the thermodynamic Bethe ansatz equations For reflectionless ADE scattering theories, Phys.Lett. B253 (1991), 391394. 